\title{
Beiträge zur Oxidationskatalyse
}

\author{
Dissertation \\ zur Erlangung des Doktorgrades \\ der Mathematisch-Naturwissenschaftlichen Fakultäten \\ der Georg-August-Universität \\ zu Göttingen
}

vorgelegt von

Diplom Chemiker

Stefan Dietrich

aus Ratingen

Göttingen 1998 
D 7

Referent:

Prof. Dr. Dr. h.c. mult. H. W. Roesky

Korreferent:

Prof. Dr. M. Buback

Tag der mündlichen Prüfung: 04.11.1998 
Meiner lieben Frau Manuela 
Die vorliegende Arbeit wurde in der Zeit vom 1.11.1995 bis zum 1.7.1998 unter der Leitung von Prof. Dr. Dr. h.c. mult. H. W. Roesky im Institut für Anorganische Chemie der Universität Göttingen durchgeführt.

Mein besonderer Dank gebührt meinem Doktorvater Prof. Dr. Dr. h.c. mult. H. W. Roesky

für seine Initiative bei der Auswahl der Themen und der Kooperationspartner aus der chemischen Industrie, für die Bereitstellung der notwendigen Mittel zur Durchführung der experimentellen Arbeiten sowie für seine finanzielle Unterstützung insbesondere durch die Vermittlung von Stipendien.

Der Otto-Vahlbruch Stiftung sowie der DFG danke ich für die großzügige Vergabe der Stipendien, die mir die Promotion ermöglichten. Den Mitgliedern des DFG Graduiertenkollegs „Kinetik und Selektivität chemischer Reaktionen in verdichteter fluider Phase" danke ich zudem für die anregenden Diskussionen und die gute Zusammenarbeit.

Der Zentralforschung der Firma Hoechst AG sowie unserem Ansprechpartner Herrn Dr. R. Schulz möchte ich für die Gelegenheit zur Präsentation unserer Daten im Rahmen des Verbundprojekts Katalyse danken. Das Projekt wurde leider unerwartet im Dezember 1997 eingestellt.

Darüber hinaus bin ich allen Mitarbeiterinnen und Mitarbeitern der Serviceabteilungen sowie meinen Abteilungskolleginnen und Arbeitskollegen für die gute Zusammenarbeit und das ausgezeichnete Arbeitsklima zu Dank verpflichtet. 


\section{Verzeichnis der verwendeten Abkürzungen}
Abb. Abbildung
acac Acetylaceton
Ber. berechnet
Cp* Pentamethylcyclopentadienyl
$\delta \quad$ chemische Verschiebung [ppm]
EI Elektronenstoßionisation
Gef. gefunden
GC Gaschromatograph
h Stunde(n)
HOMO Highest Occupied Molecular Orbital
$i$-Buten Isobuten
IR Infrarot
kl. klein
$\mathrm{M}^{+} \quad$ Molekülion
Me Methyl
Min. Minute
MS Massenspektrum
m/z Verhältnis Masse/Ladung
n-Hexan normales (unverzweigtes) Hexan
NHPI N-Hydroxyphthalimid
NMR Kernmagnetische Resonanz (nuclear magnetic resonance)
OAc Acetatrest
$\tilde{v} \quad$ Wellenzahl $\left[\mathrm{cm}^{-1}\right]$
PEG xxx Polyethylenglykol der mittleren Molmasse xxx g/mol
$\mathrm{Ph} \quad$ Phenyl
$\mathrm{R}, \mathrm{R}^{\prime} \quad$ organischer Rest 
RT Raumtemperatur

$t$-Bu tert-Butyl

THF Tetrahydrofuran

TMS Tetramethylsilan 


\section{Inhaltsverzeichnis}

1. Einleitung $\ldots \ldots \ldots \ldots \ldots \ldots \ldots \ldots \ldots \ldots \ldots \ldots$

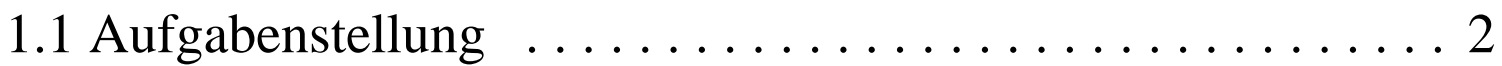

2. Theoretischer Teil $\ldots \ldots \ldots \ldots \ldots \ldots \ldots \ldots \ldots \ldots$

3. Apparativer Aufbau $\ldots \ldots \ldots \ldots \ldots \ldots \ldots \ldots$

3.1 Vorversuche ........................ . 12

3.2 Entwurf und Aufbau einer standardisierten Testapparatur . 14 3.3 Ausführung der Versuche $\ldots \ldots \ldots \ldots \ldots \ldots$

3.4 Verfahren der gaschromatographischen Untersuchung . . . 16

4. Untersuchung der verschiedenen Katalysator-Systeme ... 20

4.1 Qualitative Voruntersuchungen ............ 20

4.2 Standardisierte, quantitative Untersuchungen . . . . . . 28

5. Bewertung der empirischen Daten $\ldots \ldots \ldots \ldots \ldots \ldots$

5.1 Beurteilung der Epoxidausbeute $\ldots \ldots \ldots \ldots \ldots$

5.2 Modell zum Reaktionsmechanismus . . . . . . . . . 45

5.3 Zusammenfassung ................. 49 
6. Präparative Projekte $\ldots \ldots \ldots \ldots \ldots \ldots \ldots \ldots \ldots \ldots, 51$

6.1 Titanophosphonate $\ldots \ldots \ldots \ldots \ldots \ldots \ldots \ldots \ldots \ldots$

6.2 Wasserlösliche Metallcarbene . . . . . . . . . . . . . 58

7. Experimenteller Teil ................... 62

7.1 Allgemeine Arbeitstechniken und Meßmethoden ...... 62

7.2 Darstellung von

$\left[(\mathrm{Cp} * \mathrm{Ti})_{3}\left(t-\mathrm{BuPO}_{3}\right)_{2}\left\{t-\mathrm{BuPO}_{2}(\mathrm{OH})\right\}(\mu-\mathrm{O})_{2}\right](\mathbf{1}) \ldots \ldots \ldots \ldots 66$

7.3 Darstellung von

$\left[(\mathrm{Cp} * \mathrm{TiCl})_{2}(t-\mathrm{BuPOH})\left\{t-\mathrm{BuPO}_{2}\right\}(\mu-\mathrm{O})_{3}\right](\mathbf{2}) . \ldots \ldots \ldots \ldots 67$

7.4 Darstellung von $\left(\mathrm{C}_{10} \mathrm{H}_{16} \mathrm{Cl}_{2} \mathrm{HgN}_{4}\right)_{2}(3) \ldots \ldots \ldots \ldots .69$

8. Behandlung und Entsorgung der Abfälle .......... 72

9. GC-Daten-Tabellen zu den Abbildungen .......... 73

10. Kristallographische Daten ................. 99

11. Literaturverzeichnis $\ldots \ldots \ldots \ldots \ldots \ldots \ldots \ldots \ldots \ldots . \ldots \ldots$ 
Lebenslauf . .

Veröffentlichungen

Hochschullehrer 


\section{Einleitung}

Die Mehrzahl der Basisprodukte der industriellen Chemie werden auch heute noch durch chemische Veredelung von fossilen Bodenschätzen gewonnen. Im industriellen Maßstab sind die Alkene Propen und Ethen mengenmäßig von besonderer Bedeutung. Sie können durch thermische Spaltung von Kohlenwasserstoffen gewonnen werden und bilden die Grundlage für eine Vielzahl von anderen Basisprodukten. Neben der Polymerisation und Hydroformylierung stellt die Epoxidation eine der besonders wichtigen Veredelungsstufen dar.

Seit der ersten Hälfte des Jahrhunderts werden die Alkene zur großtechnischen Darstellung von Epoxiden mit hypochloriger Säure oxidiert und anschließend mit Kalkmilch zum Epoxid dehydrohalogeniert (Chlorhydrinverfahren). Der Chlorverlust und die Bildung großer Mengen wertloser Calciumdichloridlösungen haben das Verfahren jedoch schnell unrentabel werden lassen. ${ }^{[1]}$

Während im Falle des Ethens ein Ersatzverfahren zur direkten Oxidation mit molekularem Sauerstoff am Silberkontakt entwickelt werden konnte, bildet das Propen unter ähnlichen Bedingungen Acrolein als Oxidationsprodukt des allylständigen Wasserstoffs der Methylgruppe. Da die Nutzung der freigewordenen Kapazitäten der Ethen-Chlorhydrin-Anlagen die Wirtschaftlichkeit der Propenoxiddarstellung nur kurzzeitig verbesserte, suchte man weiter nach einem billigeren, direkten und chlorfreien Epoxidierungsverfahren für Propen. 
Erst in den 70er Jahren wurde durch die indirekte Oxidation von Propen über organische Hydroperoxide (Oxiran-Prozeß) eine chlorfreie, großtechnische Propenoxiddarstellung möglich. Durch die bei diesen Verfahren gebildeten organischen Nebenprodukte wie Styren, tert-Butanol oder iso-Buten ist die Rentabilität des Prozesses jedoch stark vom Bedarf an diesen Produkten abhängig. ${ }^{[1]}$

Die weitere Erforschung des Oxiran-Verfahrens ermöglichte die Entwicklung verschiedenster Katalysatoren, welche heute den umweltfreundlichen Einsatz von Wasserstoffperoxid als Oxidationsmittel erlauben.

Für die direkte Oxidation von Propen mit molekularem Sauerstoff ist jedoch trotz intensiver Forschung bis zum heutigen Tage kein Verfahren bekannt geworden, welches Eingang in eine wirtschaftliche, großtechnische Produktion von Propenoxid gefunden hätte.

\subsection{Aufgabenstellung}

Ziel der vorliegenden Arbeit war es, neue katalytische Verfahren zur Oxidation organischer Verbindungen zu entwickeln und zu untersuchen. Ein besonderer Schwerpunkt wurde dabei auf die Untersuchung der katalytischen Epoxidation von Alkenen mit molekularem Sauerstoff gelegt. 


\section{Theoretischer Teil}

Die Reaktion von Sauerstoff mit organischen Molekülen ist im allgemeinen ein thermodynamisch günstiger Vorgang, wie man leicht am exothermen Verbrennungsprozeß sehen kann. Trotzdem kommt es normalerweise bei Raumtemperatur nicht zu spontanen Oxidationen, da eine kinetische Hinderung besteht.

Das Sauerstoffmolekül befindet sich unter normalen Bedingungen in einem reaktionsträgen Triplettgrundzustand ${ }^{3} \sum_{\mathrm{g}}{ }^{-}$mit zwei ungepaarten Elektronen in den entarteten, antibindenden $\pi^{*}$-Molekülorbitalen. Die direkte Reaktion mit dem HOMO eines organischen Moleküls im Singulettzustand würde das Prinzip von der Erhaltung der Spinsymmetrie verletzen („Symmetrie-verbotener Prozeß“). Um die Oxidationsreaktion zu ermöglichen, muß das Sauerstoffmolekül in einem vorgelagerten Reaktionsschritt aktiviert werden.

Die Aktivierung kann zum Beispiel durch Reduktion zum Peroxid-Ion $\mathrm{O}_{2}{ }^{2-}$ erfolgen. Der Sauerstoff weist in Hydroperoxiden durch die vier gepaarten Elektronen einen Singulettzustand auf und kann so direkt mit dem Alken reagieren.

Durch Zufuhr von 92 kJ/mol Energie z.B. in Form von Wärme oder Lichtenergie kann auch das nichtoxidierte Sauerstoffmolekül in einen Singulettzustand versetzt werden. Es erreicht dann den reaktiven ersten angeregten ${ }^{1} \Delta_{\mathrm{g}}$-Zustand mit zwei gepaarten Elektronen in einem antibindenden $\pi^{*}$-Orbital. ${ }^{[2]}$ 
Da die Energiezufuhr die Reaktivität unspezifisch erhöht und so die Selektivität senkt, kommt es jedoch auf diesem Weg sehr oft zur vollständigen, oftmals explosionsartigen Oxidation.

Ein weiterer Weg, die kinetische Hemmung zu überwinden, ist die Multiplizitätsanpassung durch Wechselwirkungen des Sauerstoffmoleküls mit einem paramagnetischen (d.h. mit ungepaarten Elektronen versehenen) Metall. ${ }^{[2]}$ Neben den beschriebenen Prozessen sind Radikalreaktionen möglich, die dem Symmetrieverbot nicht unterliegen und somit keine kinetische Hemmung aufweisen. Radikalische Oxidationen bilden die Grundlage für die zu beobachtende langsame Autooxidation, sind aber auch in der Flüssigphasenoxidation $^{[3]}$ und bei Reaktionen in der Gasphase beteiligt, die ebenfalls zu den gewünschten Epoxiden führen können.

Da das gesamte Leben auf der Erde auf Redoxprozessen beruht, lag es nahe, natürliche Vorbilder für die gewünschten Reaktionen zu suchen und aufzuklären. Dieser biomimetische Ansatz führte in der Forschung über die Untersuchungen an Hämoglobin, Cytochromen und Peroxidasen zu dem weiten Feld der sogenannten "Gif " Chemie mit Eisen als zentralem Metall. ${ }^{[4]}$

Die Technik konzentrierte sich weiter auf die Übertragung peroxidischen Sauerstoffs. Mit dem preiswerten, umweltfreundlichen und in großen Mengen verfügbaren Wasserstoffperoxid steht dafür ein geeignetes Oxidationsmittel zur Verfügung. Aber auch die in situ Bildung von peroxidischem Sauerstoff durch die Reaktion von Kupfer-(I)-Komplexen mit Sauerstoff oder die katalytische Oxidation in Gegenwart von Aldehyden wurde untersucht. ${ }^{[5]}$ 
Vor fast zwanzig Jahren entdeckte man, daß auch Titanosilicate und Zeolithe unter milden Bedingungen die Epoxidation von Propen mit Wasserstoffperoxid ermöglichen. Die Darstellung und Verwendung von Titanosilicaten verschiedenster Art zu Propenoxidation ist in der Literatur detailliert beschrieben ${ }^{[6-7]}$ und zum Teil patentiert. ${ }^{[8]}$

So wird die industrielle Oxidation von Propen zu Propenoxid in großem Maßstab mit Wasserstoffperoxid an Titanosilicaliten (TS1) bei Drücken von 3 bar oder mit molekularem Sauerstoff über in situ gebildete Hydroperoxide bei 15 - 65 bar durchgeführt. Durch die Verwendung der Hydroperoxide ist die Bildung von Nebenprodukten jedoch nicht zu vermeiden.

Im Falle des Wasserstoffperoxids ist das Nebenprodukt Wasser, welches zu Nebenreaktionen und Korrosionsproblemen führt. Außerdem kann Wasserstoffperoxid nicht in hohen Konzentrationen stabilisiert werden, so daß bei der Reaktionsführung große Volumina an Wasser komprimiert, erhitzt, abgekühlt und abgetrennt werden müssen, ohne daß sie für die Epoxidierungs-reaktion von Nutzen wären.

Um die direkte Oxidation mit molekularem Sauerstoff zu ermöglichen, wurden zu Beginn der Arbeit zwei unterschiedliche Wege in Betracht gezogen, einerseits die oben beschriebene katalytische Wirkung von Metallen zur Aktivierung des Sauerstoffes zu nutzen und andererseits die radikalische Autooxidation zu fördern. 
In vielen technischen Oxidationsverfahren mit molekularem Sauerstoff werden Vanadiumverbindungen als Katalysatoren verwendet (z.B. für die $\mathrm{SO}_{2}$ Oxidation ${ }^{[9]}$ ). Ihr katalytischer Effekt ist auch für die Katalyse der Epoxidation von Alkenen mit Hydroperoxiden bekannt: ${ }^{[10 a]}$

Abb. 1

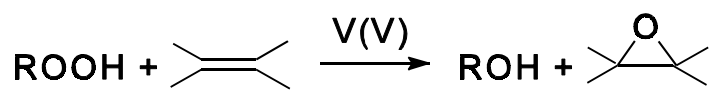

Hierbei kommt es nach Mimoun et al. primär nicht zu einer Metall-AlkenWechselwirkung sondern zu einer Wechselwirkung zwischen einer Metalloxospezies und dem aktivierten Sauerstoff: ${ }^{[10 b]}$

Abb. 2

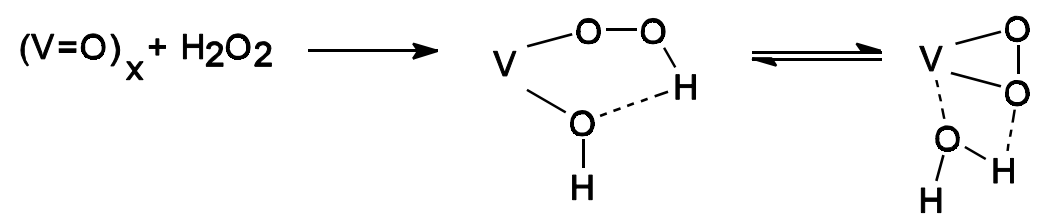

Die dreigliedrige V(V)-Peroxospezies kann nun unter Reduktion des Vanadiums zu einem V(IV)-Peroxoradikal öffnen, welches dann ein Sauerstoffatom radikalisch auf ein Alken überträgt und die $\mathrm{V}(\mathrm{V})$-Oxospezies zurückbildet. ${ }^{[1]}$

Da Vanadiumverbindungen zudem auch mit molekularem Sauerstoff wechselwirken können und dabei dessen Reduktionspotential beeinflussen, sollten sie den Ausgangspunkt der Untersuchungen bilden. ${ }^{[12]}$

Abb. 3

$$
\mathrm{V}(\mathrm{IV})+\mathrm{O}_{2} \longrightarrow \mathrm{V}(\mathrm{IV}) \cdots \cdots \mathrm{O}_{2} \stackrel{\mathrm{e}^{-}}{\longrightarrow} \mathrm{V}(\mathrm{V})-\mathrm{O}_{2}{ }^{2-}
$$


Um grundsätzliche Erkenntnisse zu gewinnen, wurden eine Reihe von in situ dargestellten Systemen unter verschiedenen Bedingungen auf ihr Epoxidationsvermögen untersucht. Eine Charakterisierung der einzelnen Spezies fand nicht statt, wenn keine nennenswerte Epoxidbildung nachzuweisen war.

Die als aktive Spezies für die Sauerstoffübertragung diskutierten $\mathrm{VO}_{x}$ Verbindungen sollten analog $\mathrm{zu}$ vielen biologisch-aktiven Systemen über Bindungen an Stickstoffatome stabilisiert werden. Als Liganden wurden deshalb in einer ersten Versuchsreihe $\alpha$-Hydroxyiminopropiophenon, alkyllithiierte Carbodiimide, Diaminodisilane, Alkyldiamine, Chinoline, Triazine usw. synthetisiert und eingesetzt.

Abb. 4

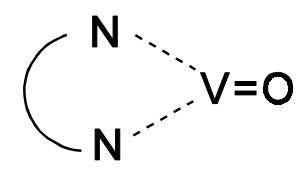

In einer weiteren Versuchsreihe sollte das Metallzentrum über Sauerstoffatome gebunden werden. Hierzu wurden Diphenylsilandiol und Polyethylenglykole (Tetraethylenglykol, PEG 400) als Stabilisator oder z.B. tert-Butoxy- und Triphenylsiloxygruppen als Liganden eingesetzt.

Abb. 5<smiles>O=[V]O[O-]</smiles>

$(\mathrm{RO})_{3} \mathrm{~V}=\mathrm{O}$ 
Es zeigte sich, daß die Epoxidausbeute von der unter Autooxidationsbedingungen zu erreichenden nicht zu unterscheiden war. Deshalb wurden den Systemen zusätzlich Kupfer-(I)-salze zugegeben, um Elektronen für die Reduktion des Sauerstoffs (Abb. 3) zur Verfügung zu stellen und die Bildung von Peroxidionen zu fördern.

Abb. 6

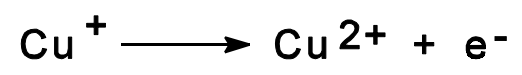

Im Verlauf der Arbeit sind zahlreiche weitere Vanadiumverbindungen und Gemische getestet worden, ohne daß nennenswerte Epoxidausbeuten ermittelt werden konnten. Das Screening wurde daraufhin auf die Synthese von einigen Platin und Molybdän-Oxo- bzw. Peroxo-Systemen ausgedehnt. Zudem wurden in Analogie zu den Titanosilicaten gesinterte Titanaluminate dargestellt und auf Epoxidationsaktivität untersucht:

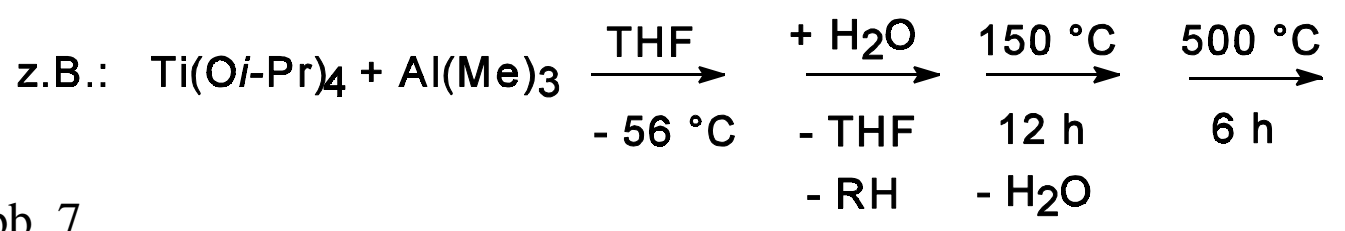

Abb. 7

Da die Vorgehensweise zu keinem Erfolg führte und auch entgegen aller Erwartung keinen Ansatzpunkt für eine gezieltere Vorgehensweise offenbarte, wurden die Screening-Versuche endgültig eingestellt. 
Der Ansatz, binäre Katalysatorsysteme zu entwickeln, in denen ein System den Sauerstoff aktiviert und ein zweites System die Übertragung des aktivierten Sauerstoffs auf das Alken übernimmt, wurde von Blanc et al. schon 1974 beschrieben. ${ }^{[13]}$ Die Oxidation sollte demnach in drei Stufen verlaufen. Zuerst wird der Sauerstoff katalytisch aktiviert, dann von einem zweiten Katalysator übertragen und schließlich wird das Katalysatorensystem regeneriert:

$$
\begin{array}{r}
\mathrm{M}_{\mathrm{A}}+\mathrm{O}_{2} \longrightarrow\left(\mathrm{M}_{A} * \mathrm{O}_{2}\right) \stackrel{\mathrm{M}_{\mathrm{B}}}{\longrightarrow}\left(\mathrm{M}_{\mathrm{B}} * \mathrm{O}_{2}\right)+\mathrm{M}_{A} \\
\left(\mathrm{M}_{\mathrm{B}} * \mathrm{O}_{2}\right)+\text { Substrat } \longrightarrow \text { Produkte }+\mathrm{M}_{B}
\end{array}
$$

Abb. 8

Versuche, komplette Katalysezyklen auf Basis von Kupfer und Chinhydron, sowie auf Basis von Tetrakis[triphenylphosphan]-Platin mit Palladiumsalzen zu ermöglichen, führten jedoch in der Regel zur Fällung des reduzierten Metalls.

Da sich metallisches Kupfer und Kupferchloride in den Vorversuchen nicht als Aktivatoren für Sauerstoff behaupten konnten, wurde nach Alternativen gesucht. 
Bereits 1983 beschreiben Masui et al. den Einsatz eines organischen Hydroxylamins zur Sauerstoffaktivierung in der elektrochemischen Oxidationschemie. ${ }^{[14]}$

In den darauf folgenden Jahren publizierte die Gruppe erste Ergebnisse der Alken-Epoxidation mit molekularem Sauerstoff an speziellen metallorganischen Verbindungen unter Zusatz von N-Hydroxyphthalimid als Cokatalysator. ${ }^{[15]}$ Der Einsatz anderer Amine und Hydroxylamine in der Oxidationskatalyse ist ebenfalls bekannt. ${ }^{[16]}$

Abb. 9

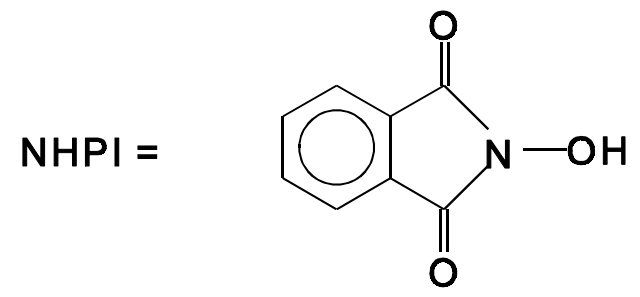

Im Jahr 1996 berichten Ishii et al. auf dem " $6^{\text {th }}$ International Symposium on the Activation of Dioxygen and Homogeneous Catalytic Oxidation" (April 14-19, 1996, Noordwijkerhout, The Netherlands) erstmals über die katalytische Oxidation von Alkanen mit molekularem Sauerstoff durch eine Kombination von Cobaltacetylacetonaten mit NHPI. ${ }^{[17]}$

Die katalytische Epoxidation mit molekularem Sauerstoff durch MetallAcetylacetonat-Katalysatoren war zwar für sich genommen bereits bekannt, verlief jedoch nur in Kombination mit Aldehyden als Coreduktanten und somit letztendlich nach dem Prilezaev Mechanismus über sauerstoffübertragende Peroxosäuren. ${ }^{[18]}$

Abb. 10

$\mathrm{RCHO}+\mathrm{O}_{2} \stackrel{\text { Kat. }}{\longrightarrow} \mathrm{RC}(\mathrm{O}) \mathrm{OOH}$ 
Da der von Ishii et al. beschriebenen Alkanoxidation ein radikalischer Mechanismus zugrunde liegt, ${ }^{[19]}$

Abb. 11<smiles>[O]N1C(=O)c2ccc3c(cccc3c2O)C1=O</smiles>

laufen die eingangs beschriebenen Wege zur Ermöglichung einer Direktoxidation hier in idealer Weise zusammen.

Die Kombinationen von verschiedenen Metallsalzen einschließlich der Metallacetylacetonate mit NHPI stellen jeweils ein binäres Katalysator-System dar, welches zum einen die katalytische Sauerstoffaktivierung und Übertragung ermöglicht und zum anderen die radikalische Autooxidation fördert.

Ausgehend von diesem Modellansatz sollten nun empirische Daten zur NHPI gestützten Alkenepoxidation mit molekularem Sauerstoff gesammelt werden, um Gesetzmäßigkeiten und mechanistische Erklärungen zu finden.

Aufgrund dieser Ergebnisse sollte dann ein zukünftiges, binäres, katalytisch aktives Vanadylsystem entwickelt werden, welches die Epoxidation von Propen mit hoher Ausbeute und großer Selektivität ermöglicht. 


\section{Apparativer Aufbau}

\subsection{Vorversuche}

Wegen der hohen Explosionsgefahr von Gemischen niederer n-Alkene mit molekularem Sauerstoff und unter Berücksichtigung des nötigen apparativen Aufwands zur Durchführung von Reaktionen mit gasförmigen Edukten, konnte die direkte Untersuchung von Propen nicht in Betracht gezogen werden.

Als Modellsubstanz mußte deshalb ein Alken dienen, welches unter normalen Bedingungen im flüssigen Aggregatzustand vorliegt. Über die Flüssigphasenoxidation von flüssigen „Olefinkohlenwasserstoffen“ liegen zudem bereits zahlreiche Untersuchungen von Schnurpfeil et al. vor. ${ }^{[20]}$

Die Doppelbindung sollte darüber hinaus, analog zu Propen, nicht aktiviert sein, so daß cyclische (Ringspannung) oder substituierte Alkene ausgeschlossen wurden. Man wählte daher 1-Octen als Substrat, dessen Autooxidationsvorgänge zudem von Pritzkow et al. beschrieben sind. ${ }^{[21]}$

Um den apparativen Aufwand und die Explosionsgefahr auch im weiteren Verlauf gering zu halten, wurde die Ermittlung der katalytischen Aktivität bei normalem Druck durchgeführt.

Der erste Versuchsaufbau bestand entsprechend den Vorgaben aus einem Zweihals-Rundkolben mit Rückflußkühler und Magnetrührstab. Das Substrat wurde (gegebenenfalls mit Lösungsmittel) in den Kolben gegeben und auf die festgelegte Reaktionstemperatur erhitzt. Mit Hilfe eines Gaseinleitungsrohres wurde Sauerstoff aus einer Druckbombe durch die Lösung geperlt. Die Reaktionen wurden unter verschiedenen Bedingungen durchgeführt. 
Anschließend wurde der flüssige, gaschromatographisch flüchtige Teil der Reaktionslösung mittels gaschromatographischer Methoden untersucht.

Die Reaktionsdurchführung zeigte grundsätzliche Probleme insbesondere hinsichtlich der Reproduzierbarkeit der durchgeführten Messungen. Die Regulierung der Sauerstoffmenge und der Substanzaustrag mit dem Sauerstoffstrom bereitete größere Schwierigkeiten. Zudem zeigte sich, daß sowohl die Rührgeschwindigkeit als auch die Rührstabgröße nicht zu vernachlässigen waren. Der zeitliche Verlauf der Aufheiz- und Abkühlphase sowie das Verhältnis von Volumen zu Oberfläche der Reaktionslösung stellten weitere einflußreiche Parameter dar.

Die Problematik der Regulierung des Sauerstoffstroms und der damit verbundene Substanzaustrag war leicht zu umgehen, indem man die Reaktion in einer stationären Sauerstoffatmosphäre durchführte. Zu diesem Zweck spülte man das Reaktionsgefäß mit Sauerstoff und schloß den Gasraum mit einem mit Sauerstoff gefüllten Gummiballon auf dem Rückflußkühler ab.

Die anderen Parameter konnten jedoch nur durch einen vereinheitlichten Versuchsaufbau konstant gehalten werden. Um vergleichbare und reproduzierbare Werte zu bestimmen, wurde daher eine Testapparatur entwickelt und aufgebaut. 


\subsection{Entwurf und Aufbau einer standardisierten Testapparatur}

Die Apparatur besteht aus acht baugleichen Metallheizblöcken, die über einen Verteiler mit Motoröl (als Wärmetauscherflüssigkeit) gleichmäßig beheizt werden. Das Motoröl wird dabei in einem großvolumigen, temperierten Heizbad erwärmt und umgepumpt.

In den Heizblöcken befindet sich jeweils ein standardisierter $25 \mathrm{ml}$ Zweihalskolben mit aufgesetztem Rückflußkühler. Auf dem Rückflußkühler befindet sich ein Gasleitungssystem, welches mit einem Gummiballon gegen Druckschwankungen gesichert ist. Aus einer Druckbombe kann Sauerstoff in das Leitungssystem und die Reaktionsapparatur geleitet werden. Über verschiedene Ventile kann dann wahlweise der Sauerstoff zum Spülen über die Reaktionsapparatur geleitet werden oder mit dem Gummiballon ein stationärer Druck von ca. 1 bar in der Apparatur aufgebaut werden. Außerdem kann man jedes Reaktionsgefäß mit einem graduierten, mit gesättigter Salzlösung gefüllten U-förmigen Glasrohr verschließen. Auf diese Weise läßt sich die Sauerstoffaufnahme sowohl qualitativ als auch quantitativ bestimmen.

An dem Verschlußstopfen des zweiten Kolbenhalses ist eine Glasschaufel angebracht, mit deren Hilfe der Katalysator in die Reaktionslösung eingebracht wird. Die Reaktionslösungen können dann mittels $1 \mathrm{~cm}$ langer Rührstäbe im Kolben und der acht baugleichen Rührmotoren unter dem Messingheizblock gleichmäßig durchmischt werden. 


\subsection{Ausführung der Versuche}

Zur Versuchsdurchführung werden die Heizelemente auf einen einheitlichen Sollwert temperiert. In die Kolben füllt man eine vorher festgelegte Menge Lösungsmittel und 1-Octen. Dann spült man den Reaktor mit Sauerstoff. Den auf der Glasschaufel befindlichen Katalysator bringt man durch Drehen der Stopfen ein. Man führt den Heizblock unter den Kolben und wartet die aufgrund der Erwärmung stattfindende Volumenausdehnung ab. Anschließend regelt man den Sauerstoffdruck in der Höhe des Umgebungsdrucks ein (Gleichstand der Flüssigkeit in beiden Rohrschenkeln) oder hält ihn über den Gummiballon konstant.

Um dem Sauerstoff den Zugang zur Reaktionslösung zu ermöglichen, muß der Dampfdruck der verwendeten Lösungsmittel über der Reaktionslösung klein sein. Man fährt die Reaktionen daher im allgemeinen bei Temperaturen, die ca. $20{ }^{\circ} \mathrm{C}$ unter dem Siedepunkt der Reaktionslösung liegen. 


\subsection{Verfahren der gaschromatographischen Untersuchung}

Bei den ersten Versuchen stand lediglich ein alter Gaschromatograph zur Verfügung, der neben der qualitativen Aussage hinsichtlich der Epoxidausbeute nur eine grobe quantitative Abschätzung ermöglichte, da sich der Gasstrom nicht mehr zuverlässig einregeln ließ.

Die Verwendung eines inneren Standards führte häufig zu Fehlmessungen, da keine unter den radikalischen Reaktionsbedingungen inerte Standardsubstanz gefunden werden konnte. Bei der anschließenden Integration des Chromatogramms wurden die Peaks des Lösungsmittels sowie des inneren Standards herausgerechnet (Korrektur). Als Oxidationsprodukte wurden alle Substanzen mit einer Retentionszeit größer der des 1-Octens deklariert. Ein Identifizierung der Produkte anhand der Retentionszeit durch Vergleich mit der Zielsubstanz fand nur beim Epoxid statt. Da die aufgeführten Werte direkt aus den GC Meßergebnissen ohne inneren Standard und ohne Berücksichtigung der beschriebenen Probleme hinsichtlich der Reproduzierbarkeit bestimmt wurden, besitzen die im ersten Auswertungsteil angegebenen Prozentwerte lediglich einen relativen Charakter, der den qualitativen Vergleich erleichtern soll.

In den später durchgeführten Versuchen mit Hilfe eines voll funktionsfähigen Gaschromatographen wurde eine genau definierte Menge Substrat (1-Octen) eingewogen. Erst nach der Reaktion setzte man dann der Reaktionsmischung eine ebenfalls genau eingewogene Menge eines inneren Standards ( $n$-Nonan) im Verhältnis 1:2 zur verwendeten Menge 1-Octen zu. Nach Verdünnung mit einer konstanten Menge $n$-Pentan wurde die Probe gaschromatographisch analysiert. 
Die Integration des Chromatogramms erfolgt mit Hilfe zuvor erstellter Eichkurven für die Responseverhältnisse 1-Octen / $n$-Nonan, 1-Octenoxid / $n$-Nonan und anderer.

Die verwendeten Begriffe Umsatz, Ausbeute und Produktselektivität wurden zur Auswertung wie folgt definiert:

- Umsatz: Der Umsatz wird als der Quotient aus der Differenz zwischen eingesetzter (100\%) und gefundener Menge 1-Octen geteilt durch die eingesetzte Menge 1-Octen angegeben.

- Ausbeute: Die Ausbeute wird als der Quotient aus der Menge des erwünschten Epoxids und der eingesetzten Menge 1-Octen angegeben.

- Produktselektivität: Die Produktselektivität ist gegeben durch den Quotienten aus der Menge des gefundenen Epoxids und der Differenz zwischen eingesetztem und wiedergefundenem 1-Octen.

- Verluste: Ein großer Teil der Nebenprodukte bzw. der Epoxidfolgeprodukte fällt in nicht GC-flüchtiger Form an. Der Anteil an umgesetztem 1-Octen, der sich nicht in den mit dem GC ermittelten Oxidations-Produkten wiederfindet, wird entsprechend als Verlustfraktion bezeichnet. 
Die entstehenden Hochvakuumfraktionen und die nicht flüchtigen Rückstände werden dabei zum größten Teil auf Epoxidringöffnungsreaktionen ${ }^{[23]}$ zurückgeführt. Sie sollten dementsprechend aus Glykolderivaten und Polymeren bestehen. ${ }^{[24]}$ Gemäß unserer Definition werden die Epoxidfolgeprodukte der Epoxidausbeute nicht zugerechnet.

Es sei darauf aufmerksam gemacht, daß in der Literatur verschiedene Methoden für die Auswertung verwendet werden, die zum Teil auf die Arbeiten von Schnurpfeil et al. ${ }^{[20]}$ zurückgehen. Herrmann et al. ${ }^{[22]}$ verwenden z.B. die folgenden Definitionen:

„1.) Umsatz: Darunter ist der Quotient aus der Summe aller Reaktionsprodukte mit einem Siedepunkt höher als 1-Octen und dem nicht umgesetzten 1-Octen zu verstehen.

2.) Produktselektivität: Sie ist definiert als der Quotient aus der Menge des entstandenen 1-Octenoxid geteilt durch die Summe aller Reaktionsprodukte mit einem Siedepunkt höher als 1-Octen...."

Den Vorteil der von uns definierten Begriffe und der zur Auswertung verwendeten Methode gegenüber den aufgeführten Definitionen sehen wir in der Unabhängigkeit der Ergebnisse von den gaschromatographischen Analysebedingungen (Empfindlichkeit, Säulencharakteristik, Detektor usw.). 
Da sich der Umsatz in der von uns verwendeten Definition auf die Menge an eingesetztem Substrat bezieht und mittels eines Standards normiert wurde, werden auch Substanzverluste gaschromatographisch erfaßt.

Durch die Wahl der Bezugsgröße „nicht umgesetztes 1-Octen“ in der zitierten Definition wären theoretische Umsätze von mehr als 100 \% möglich, sobald die Menge an gefundenem Octen weniger als die Hälfte der gefundenen Gesamtproduktmenge ausmacht. Durch die Zugabe eines internen Standards zur Reaktionsmischung vor der Reaktion läßt sich zudem nicht ausschließen, daß aus dem Standard gebildete Nebenprodukte, ebenso wie z.B. Liganden-ZerfallsProdukte, die ermittelten Umsätze erhöhen. Nebenprodukte, die mittels GC nicht ermittelt werden (Carbonsäuren, Polymere u.a.), erniedrigen dagegen rechnerisch den Umsatz, erhöhen dadurch aber innerhalb der oben zitierten Literatur die Ausbeute und die Produktselektivität.

Durch die methodischen Unterschiede könnten somit höhere Ausbeuten und Selektivitäten berechnet werden. Die Angaben zum Umsatz können dabei sowohl nach oben (durch erfaßte Produkte mit einem Siedepunkt höher als 1-Octen, die nicht Folgeprodukte des 1-Octen sind) als auch nach unten (durch Produkte aus 1-Octen, die nicht gaschromatographisch erfaßt werden) abweichen. 


\section{Untersuchung der verschiedenen Katalysator-Systeme}

\subsection{Qualitative Voruntersuchungen}

Die Arbeitsgruppe von Y. Ishii oxidiert vornehmlich Cycloalkane zu den entsprechenden Alkoholen, Ketonen und Carbonsäuren. ${ }^{[17]}$ Die Reaktion verläuft dabei wie bereits erwähnt katalytisch ohne Coreduktant nach dem Mechanismus einer radikalischen Autooxidation. Bei einer Menge von 5 mmol 1-Octen kommen $10 \mathrm{~mol} \%$ NHPI und $0.5 \mathrm{~mol} \%$ des metallorganischen Cokatalysators zum Einsatz. Die Reaktion wird 6 Stunden lang bei $100{ }^{\circ} \mathrm{C}$ in Essigsäure durchgeführt. Die Essigsäure wird anschließend im Vakuum entfernt (wobei alle Edukte und Produkte mit einem ähnlichen Dampfdruck verloren gehen!).

Der verbliebene Rückstand wird mit Methanol aufgenommen, mit konz. $\mathrm{H}_{2} \mathrm{SO}_{4}$ angesäuert und bei $65{ }^{\circ} \mathrm{C} 15$ Stunden lang gerührt. Man extrahiert mit Diethylether. Zur Phasentrennung muß dann noch eine größere Menge Wasser zugegeben werden. Die etherische Phase wird über Magnesiumsulfat getrocknet und chromatographisch getrennt.

Da man bei der Epoxidierung von Alkenen die saure Hydrolyse vermeiden muß, kam in unseren Versuchen, im Gegensatz zu der von Ishii et al. beschriebenen Reaktionsführung in Essigsäure, nur ein nicht saures Lösungsmittel in Frage. Außerdem empfahl sich ein Lösungsmittel, das die direkte Untersuchung der Reaktionslösung im Gaschromatographen ohne weitere Aufarbeitung erlaubt. Anstelle von $\mathrm{Cr}(\mathrm{acac})_{3}$ wurden in den ersten Versuchen $\mathrm{Cu}(\mathrm{OAc})_{2} \cdot \mathrm{H}_{2} \mathrm{O}$, $\mathrm{Mn}(\mathrm{acac})_{2}, \mathrm{VO}\left(\mathrm{OSiPh}_{3}\right)_{3}$ sowie $\mathrm{VO}(\mathrm{acac})_{2}$ eingesetzt. Als Lösungsmittel fanden Benzonitril, tert-Butanol, Acetonitril und Dichlormethan Verwendung. 


\begin{tabular}{|c|c|c|c|c|c|}
\hline $\begin{array}{c}\text { Katalysator NHPI } \\
0.5 \mathrm{mmol} \\
\& 0.025 \mathrm{mmol} \\
\text { metallorg. } \\
\text { Cokatalysator } \\
\end{array}$ & $\begin{array}{l}\text { Umsatz zu } \\
\text { Oxidations- } \\
\text { produkten }\end{array}$ & $\begin{array}{l}\text { Epoxid- } \\
\text { anteil }\end{array}$ & $\begin{array}{l}\text { Selektivität } \\
\text { bezogen auf } \\
\text { Epoxid }\end{array}$ & $\begin{array}{l}\text { Diol- } \\
\text { anteil }\end{array}$ & $\begin{array}{c}\text { Selektivität } \\
\text { bezogen auf } \\
\text { Diol }\end{array}$ \\
\hline \multicolumn{6}{|c|}{ I. Reaktion nach Vorschrift von Ishii et al. in Essigsäure ${ }^{[17]}$} \\
\hline $\operatorname{Mn}(\operatorname{acac})_{2}$ & $4.3 \%$ & $0 \%$ & $0 \%$ & $1.2 \%$ & $26.9 \%$ \\
\hline $\mathrm{Cu}(\mathrm{OAc})_{2} \cdot \mathrm{H}_{2} \mathrm{O}$ & $16.8 \%$ & $2.1 \%$ & $12.5 \%$ & $3.6 \%$ & $21.5 \%$ \\
\hline $\mathrm{VO}\left(\mathrm{OSiPh}_{3}\right)_{3}$ & $13.0 \%$ & $1.8 \%$ & $13.9 \%$ & $6.4 \%$ & $49.5 \%$ \\
\hline \multicolumn{6}{|c|}{ II. Reaktion in Benzonitril, $6 \mathrm{~h}, 100{ }^{\circ} \mathrm{C}$ Heizbad } \\
\hline $\operatorname{Mn}(\operatorname{acac})_{2}$ & $29.3 \%$ & $0.4 \%$ & $0.0 \%$ & $0 \%$ & $0 \%$ \\
\hline \multicolumn{6}{|c|}{ III.a Reaktion in tert-Butanol, $6 \mathrm{~h}, 60^{\circ} \mathrm{C}$ Heizbad } \\
\hline $\operatorname{Mn}(\operatorname{acac})_{2}$ & $0.4 \%$ & $0 \%$ & $0 \%$ & $0 \%$ & $0 \%$ \\
\hline $\mathrm{Cu}(\mathrm{OAc})_{2} \cdot \mathrm{H}_{2} \mathrm{O}$ & $0 \%$ & $0 \%$ & $0 \%$ & $0 \%$ & $0 \%$ \\
\hline \multicolumn{6}{|c|}{ III.b Reaktion in tert-Butanol, $6 \mathrm{~h}, 60^{\circ} \mathrm{C}$ Heizbad; anschließend $6 \mathrm{~h}$, Rückfluß } \\
\hline $\operatorname{Mn}(\mathrm{acac})_{2}$ & $12.8 \%$ & $0.3 \%$ & $2.6 \%$ & $0.3 \%$ & $2.6 \%$ \\
\hline $\mathrm{Cu}(\mathrm{OAc})_{2} \cdot \mathrm{H}_{2} \mathrm{O}$ & $7.1 \%$ & $0 \%$ & $0 \%$ & $0 \%$ & $0 \%$ \\
\hline \multicolumn{6}{|c|}{ IV.a Reaktion in Acetonitril, $6 \mathrm{~h}, 80^{\circ} \mathrm{C}$ Heizbad } \\
\hline $\operatorname{Mn}(\mathrm{acac})_{2}$ & $7.0 \%$ & $0 \%$ & $0 \%$ & $0 \%$ & $0 \%$ \\
\hline $\mathrm{Cu}(\mathrm{OAc})_{2} \cdot \mathrm{H}_{2} \mathrm{O}$ & $1.1 \%$ & $0 \%$ & $0 \%$ & $0 \%$ & $0 \%$ \\
\hline $\mathrm{VO}(\mathrm{acac})_{2}$ & $2.3 \%$ & $0 \%$ & $0 \%$ & $0 \%$ & $0 \%$ \\
\hline \multicolumn{6}{|c|}{ IV.b Reaktion in Acetonitril, 6 h, $80^{\circ} \mathrm{C}$ Heizbad; anschließend 6 h, Rückfluß } \\
\hline $\operatorname{Mn}(\mathrm{acac})_{2}$ & $5.9 \%$ & $1.5 \%$ & $25.4 \%$ & $0 \%$ & $0 \%$ \\
\hline $\mathrm{Cu}(\mathrm{OAc})_{2} \cdot \mathrm{H}_{2} \mathrm{O}$ & $2.0 \%$ & $0 \%$ & $0 \%$ & $0 \%$ & $0 \%$ \\
\hline $\mathrm{VO}(\mathrm{acac})_{2}$ & $0.7 \%$ & $0 \%$ & $0 \%$ & $0 \%$ & $0 \%$ \\
\hline \multicolumn{6}{|c|}{ V. Reaktion in Dichlormethan, 6.5 h, Rückfluß } \\
\hline $\operatorname{Mn}(\mathrm{acac})_{2}$ & $0 \%$ & $0 \%$ & $0 \%$ & $0 \%$ & $0 \%$ \\
\hline $\mathrm{Cu}(\mathrm{OAc})_{2} \cdot \mathrm{H}_{2} \mathrm{O}$ & $0.8 \%$ & $0 \%$ & $0 \%$ & $0 \%$ & $0 \%$ \\
\hline $\mathrm{VO}(\mathrm{acac})_{2}$ & $0.6 \%$ & $0 \%$ & $0 \%$ & $0 \%$ & $0 \%$ \\
\hline
\end{tabular}

Abb. 12 
Aus der Tabelle (Abb. 12) geht deutlich hervor, daß unter den Reaktionsbedingungen nach Ishii et al. neben dem gewünschten Epoxid vor allem das 1,2Octendiol gebildet wird. Ändert man das Lösungsmittel, findet man bei den vorher aktiven Systemen keine Epoxid- oder Diolbildung mehr.

Lediglich das vorher nicht aktive Manganacetylacetonat bildet zum Teil noch Epoxide. Die in Essigsäure bevorzugte Diolbildung ist hingegen nicht mehr dominant. Es kann deshalb vermutet werden, daß die Essigsäure in Form von Peroxyessigsäure die Epoxidbildung ermöglicht und gleichzeitig durch saure Hydrolyse die Umwandlung in das Diol bedingt.

Die vorliegenden Ergebnisse zeigen, daß eine Epoxidation des 1-Octens mit den verwendeten Systemen prinzipiell möglich ist. Da ein positiver Einfluß der Lösungsmittel nicht zu verzeichnen war, wurden Reaktionen ohne Lösungsmittel unter verschiedensten Bedingungen durchgeführt.

Um den bestimmenden Einfluß der Reaktionsdauer auf die Epoxidbildung und die Bildung von Nebenprodukten genauer zu untersuchen, zeichnete man den zeitlichen Verlauf der Epoxidbildung auf (Abb. 13-15). 


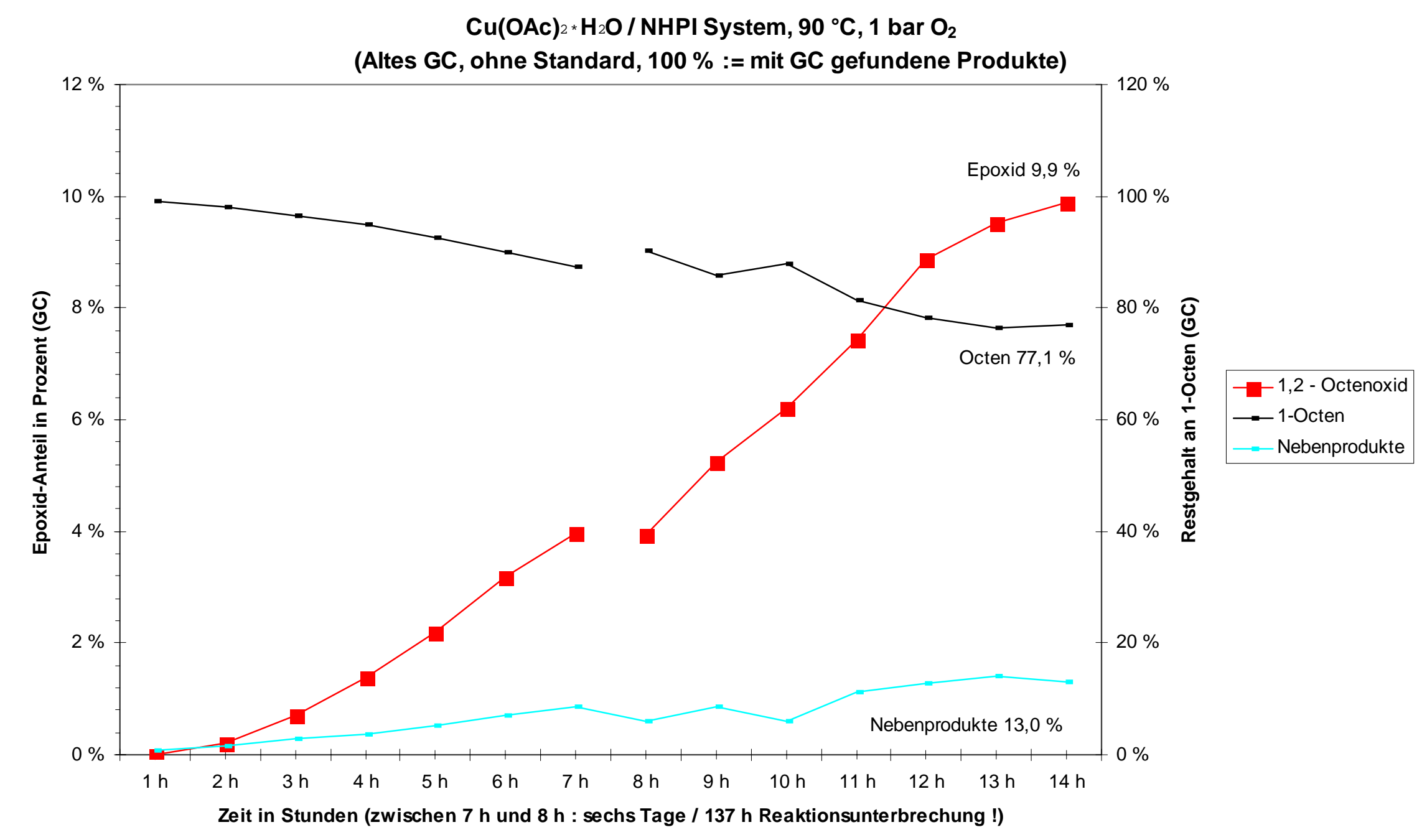

Abb. 1 
$\mathrm{Mn}(\mathrm{acac})_{2} / \mathrm{NHPI}, 90^{\circ} \mathrm{C}, 1$ bar $\mathrm{O}_{2}$

(Altes GC, ohne Standard, $100 \%:=$ mit GC gefundene Produkte)

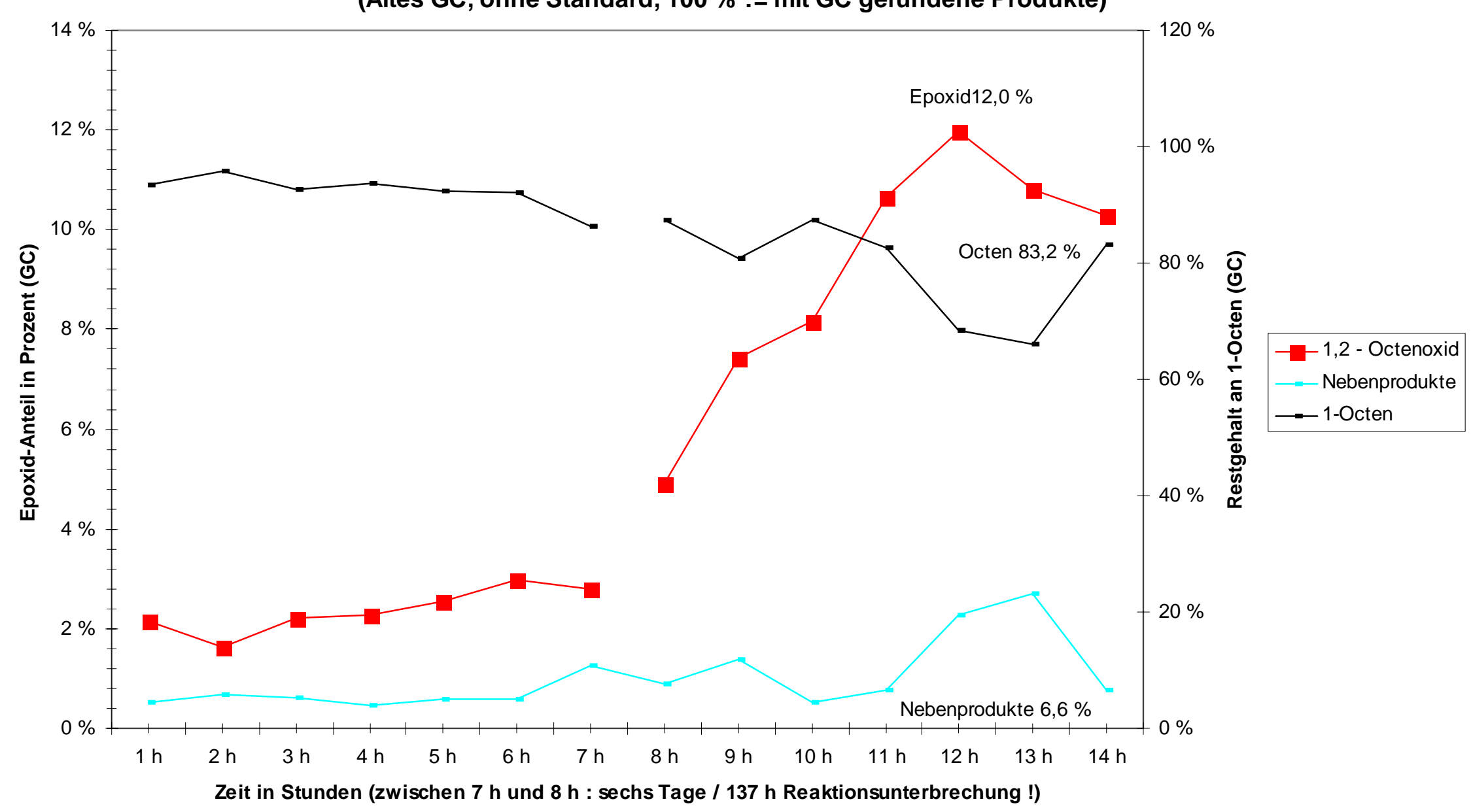

Abb. 2 


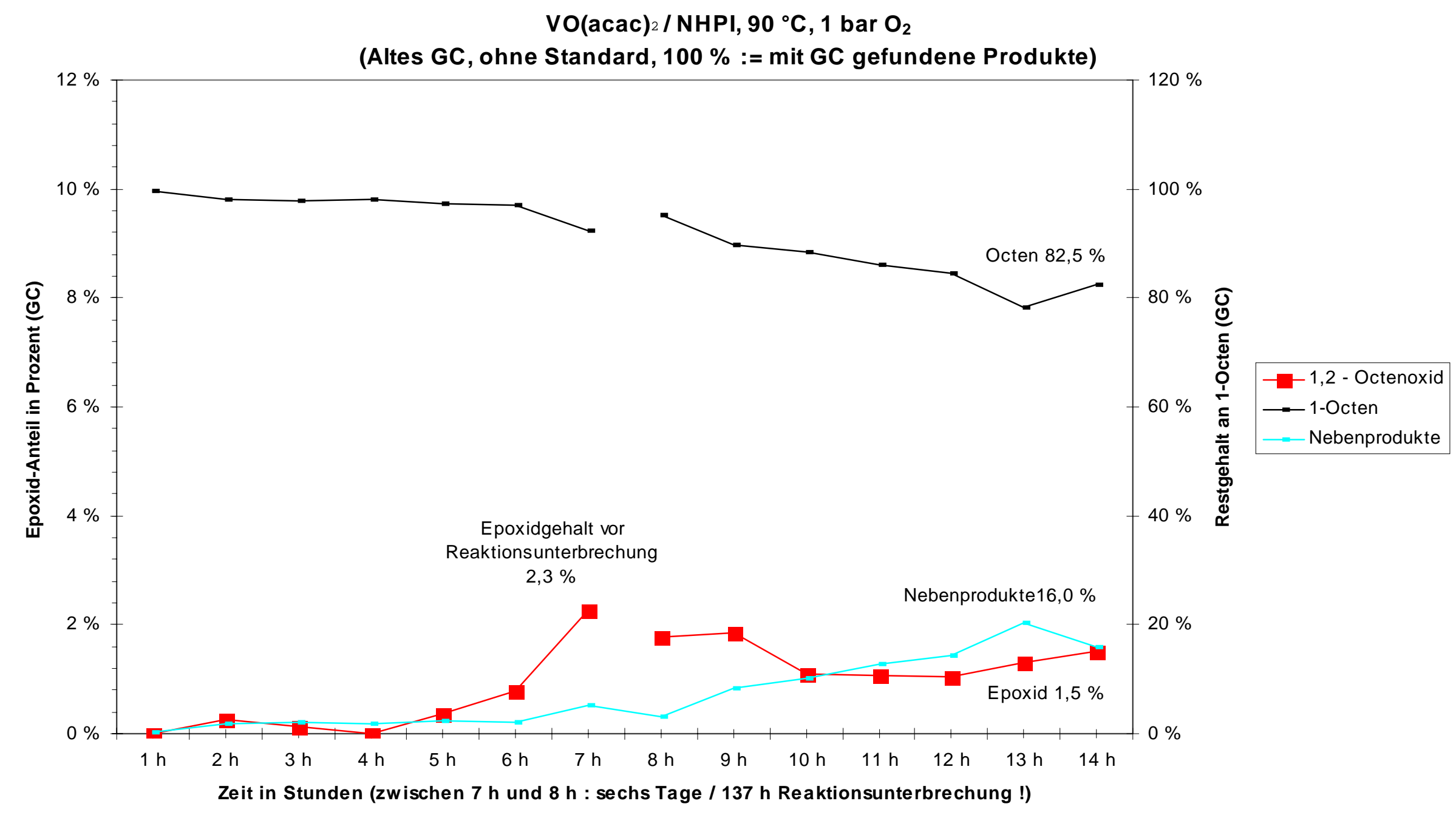

Abb. 3 
Zur Aufzeichnung des zeitlichen Verlaufs (Abb. 13-15) entnahm man dem Reaktionsgemisch im Stundenabstand jeweils eine Probe, verdünnte mit $n$-Pentan und ließ den Feststoffanteil absetzen. Die darüber stehende flüssige Phase wurde gaschromatographisch untersucht. Nach sechs Stunden Reaktionsdauer wurde dann die Sauerstoffzufuhr unterbrochen, die Reaktionsgefäße abgekühlt und geöffnet. Bei der anschließenden Auswertung war zu erkennen, daß die Epoxidbildung stetig mit der Zeit anstieg. Um festzustellen, ob eine Restaktivität des katalytischen Systems vorliegt, wurde die Reaktion nach sechs Tagen Unterbrechung fortgesetzt. Man spülte das System erneut mit Sauerstoff und brachte den Sauerstoffdruck wieder auf ca. 1 bar. Dann erhitzte man die Reaktionslösung wieder auf $90 \quad{ }^{\circ} \mathrm{C}$ und führte die Probenentnahme wie beschrieben weiter.

Die Versuchsreihen wurden unter den gleichen Bedingungen durchgeführt und untersucht (0.5 mol\% Metallsalz, $10 \mathrm{~mol} \%$ NHPI, 25 mmol 1-Octen). Obwohl in allen drei Fällen ein gleichmäßiger Octenabbau stattfindet und der Anteil der Nebenprodukte entsprechend ansteigt (sekundäre Größenachse), ist der Verlauf der Epoxidbildung uneinheitlich. Neben der Selektivität der Reaktion ist die unterschiedliche Steilheit des Kurvenverlaufs auffällig. Eine Gesetzmäßigkeit bei der Epoxidbildung oder der Restaktivität ließ sich somit noch nicht bestimmen. 
Im Fall des Kupferacetats (Abb. 13) findet man einen stetigen, linearen Anstieg der Epoxidausbeute mit der Zeit. Auch die Kurvenverläufe für den Octenabbau und die Nebenproduktbildung sind gleichmäßig. Es ist darüber hinaus zu erkennen, daß die Reaktion nach der Unterbrechung problemlos weitergeführt werden konnte. Nach ungefähr 12 Stunden Gesamtreaktionsdauer flacht die Kurve für die Epoxidausbeute dann langsam ab.

Im Mangan-System (Abb. 14) ist der Kurvenverlauf bis zur Reaktionsunterbrechung bei geringerer Epoxidbildung ebenfalls gleichmäßig. Nach der Wiederaufnahme der Reaktion ist ein starker Anstieg der Epoxidbildung zu verzeichnen. Versuche, dieses Phänomen zu reproduzieren, scheiterten, so daß für den Kurvenverlauf keine Erklärung gegeben werden kann.

Auch im Falle des Vanadylacetylacetonats (Abb. 15) sind die Kurvenverläufe für den Octenabbau und die Nebenproduktbildung gleichmäßig. Auffällig ist jedoch der exponentielle Anstieg der Epoxidbildung bis zur Reaktionsunterbrechung, der eine Induktionsphase vermuten läßt. Nach der Reaktionsunterbrechung ist ein Abbau bereits gebildeten Epoxids festzustellen. Die Fortführung der Reaktion unter Verbrauch von Octen fand dann nahezu ausschließlich zugunsten von Nebenprodukten statt. 


\subsection{Standardisierte, quantitative Untersuchungen}

Nachdem uns ein geeigneter Gaschromatograph zur quantitativen Bestimmung der Epoxidausbeute zur Verfügung stand, wurde die im Kapitel „Apparativer Aufbau" beschriebene standardisierte Testapparatur eingesetzt.

Um einen ersten qualitativen Vergleich der katalysierten Epoxidbildung mit der Autooxidation zu ermöglichen, wurde die Reaktionsdauer auf 13 bis 14 Stunden verlängert. Die Ergebnisse sind in den folgenden Darstellungen verdeutlicht.

Die erste Grafik (Abb. 16) gibt eine Gesamtübersicht geordnet nach Reaktionsdauer und Reaktionstemperatur.

In den beiden darauffolgenden Darstellungen (Abb. 17 und 18) werden die Ergebnisse für je ein Metallsalz nach der Epoxidausbeute sortiert dargestellt, um einen Vergleich der Reaktionsbedingungen zu ermöglichen.

Das letzte Schaubild dieser Reihe (Abb. 19) zeigt den Vergleich verschiedener Metalle mit dem gleichen Ligandensystem unter verschiedenen Bedingungen. 


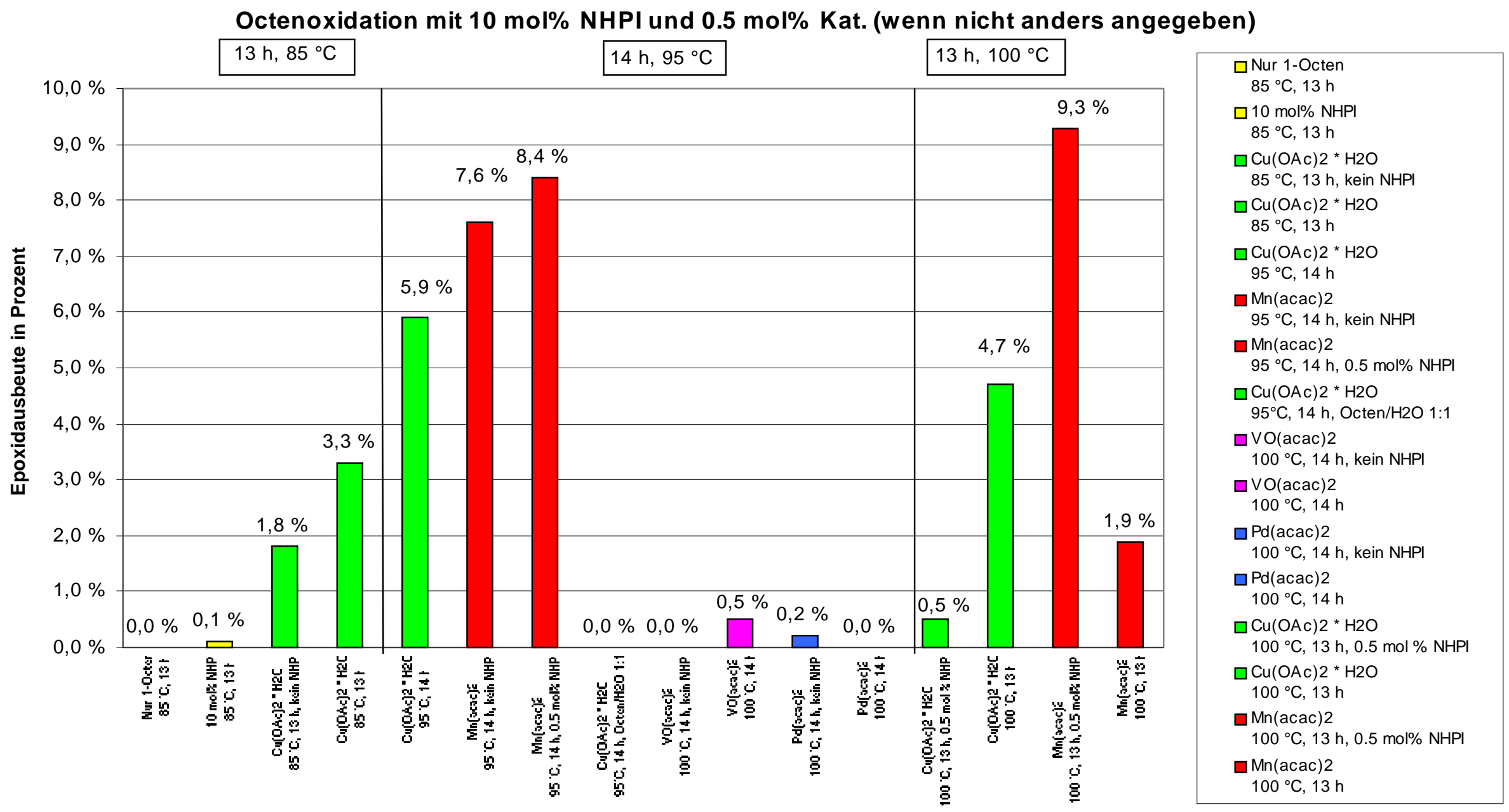

Abb. 4 


\section{Kupferacetat unter verschiedenen Bedingungen}

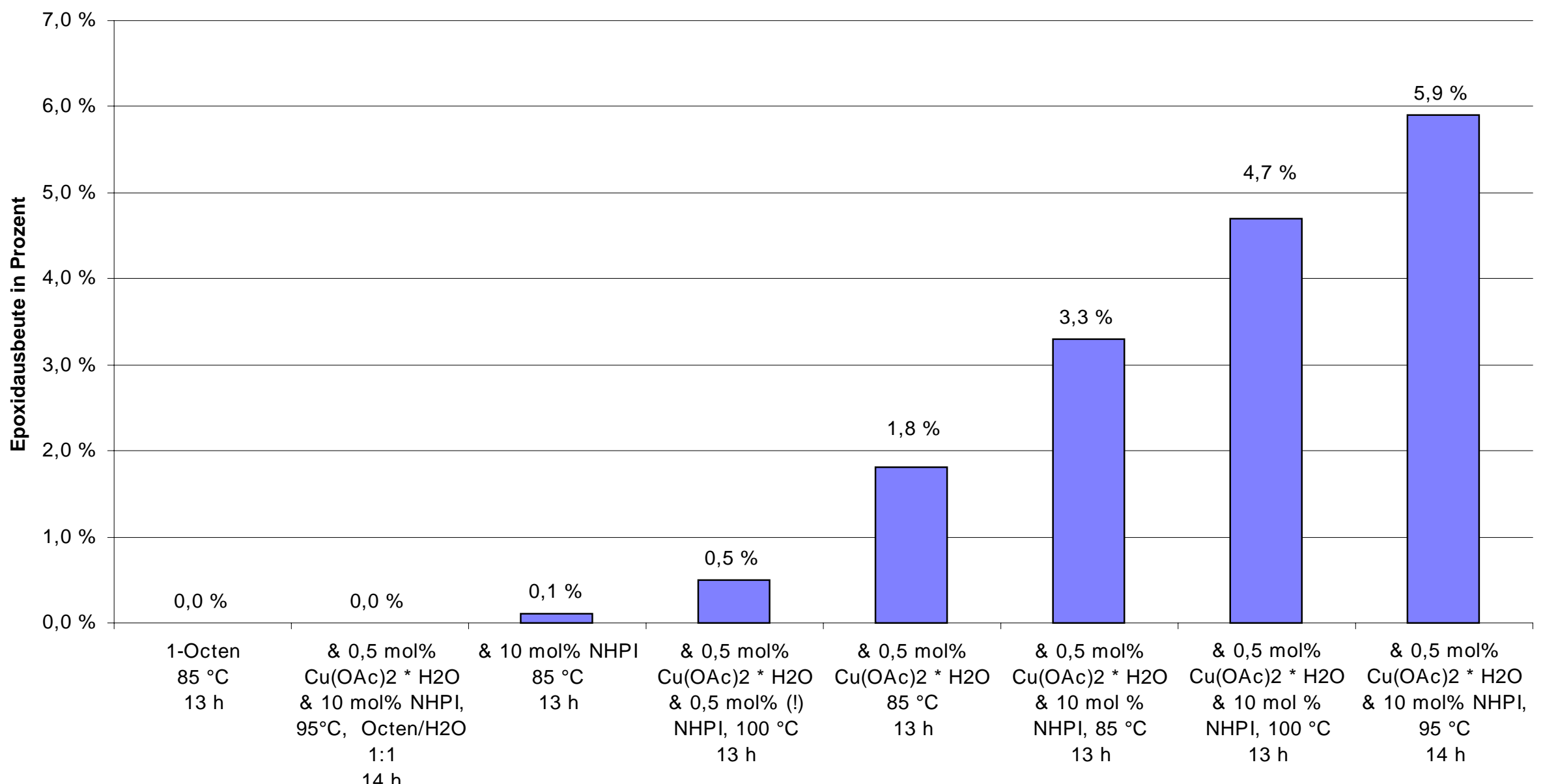

Abb. 5 
Manganacetylacetonat unter verschiedenen Bedingungen

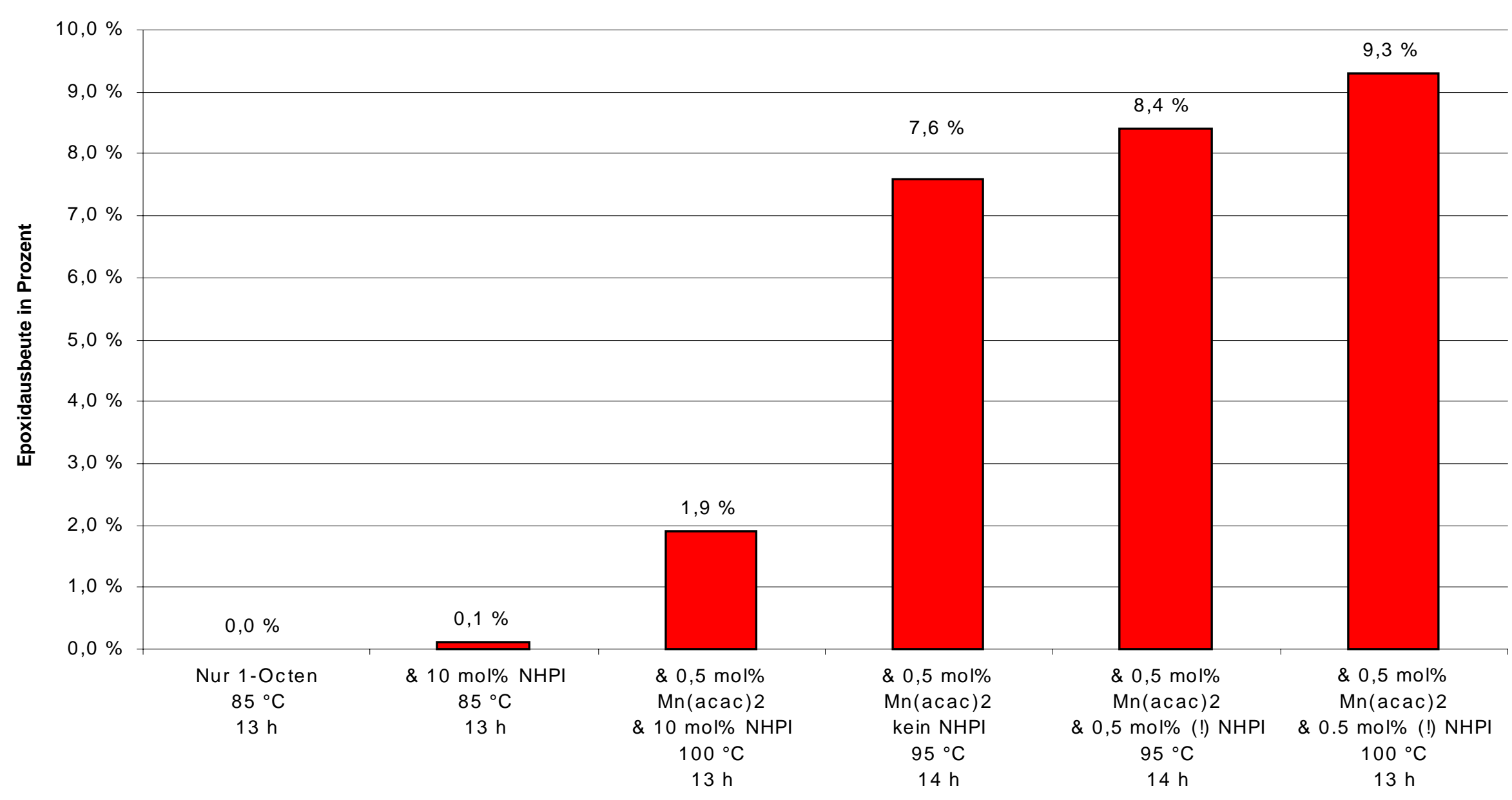

Abb. 6 
Acetylacetonate im Vergleich

$7,6 \%$

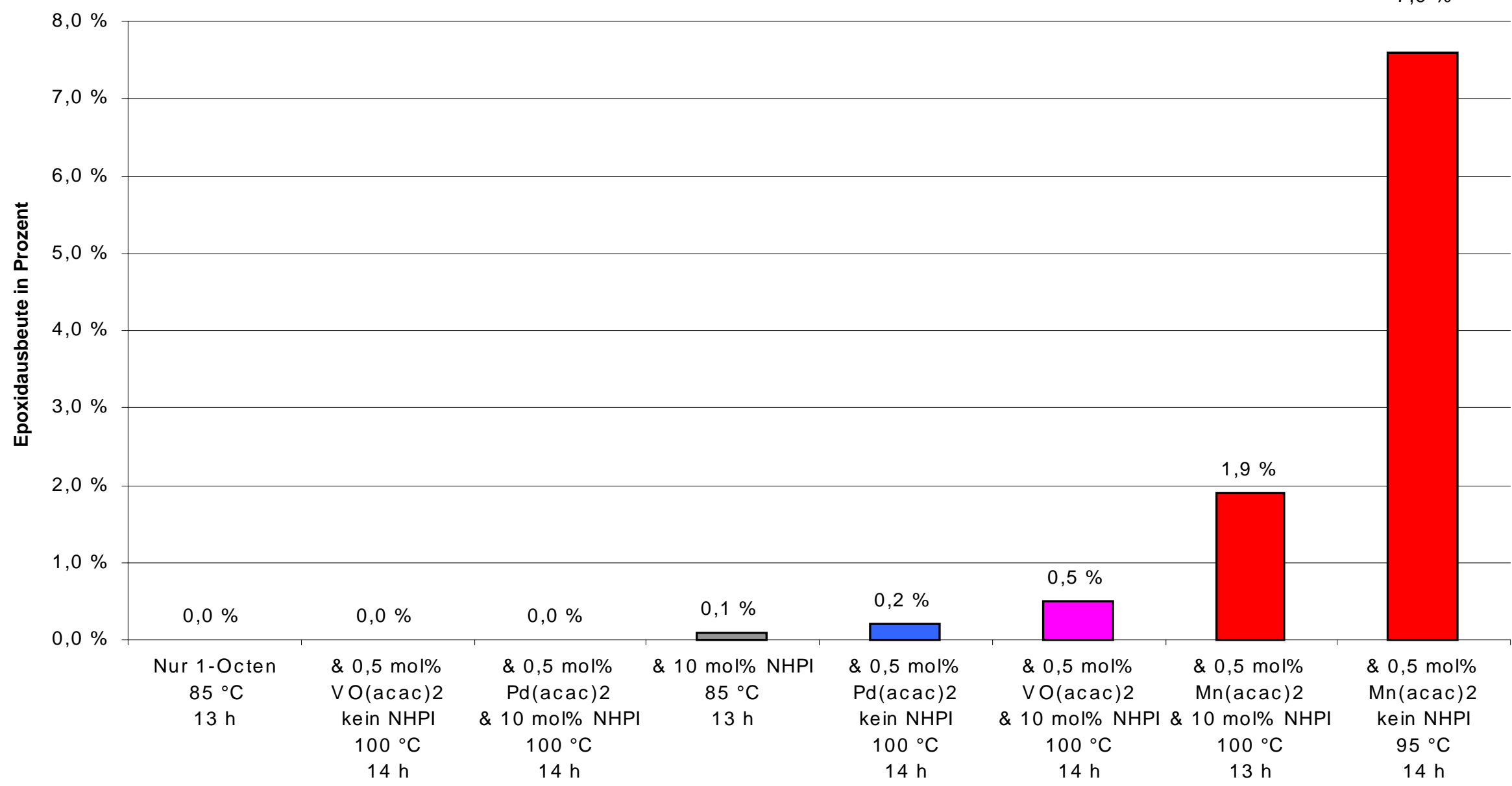

Abb. 7 
Es ist zu erkennen, daß der Einsatz einer Kombination von Metallsalz und NHPI der Autooxidation und der Katalyse mit jeweils nur einer Komponente des binären Systems hinsichtlich der Epoxidausbeute im allgemeinen überlegen ist. Die Abhängigkeit der Epoxidausbeute von der Reaktionsdauer erscheint dabei im untersuchten Rahmen signifikant höher als die Abhängigkeit von der Reaktionstemperatur (Abb. 16-18). Darüber hinaus deuten die Vorexperimente an, daß die katalytische Aktivität vermutlich vom Metallzentrum und nicht vom Ligandensystem abhängig ist (Abb. 19). Andernfalls müßte die Epoxidausbeute für alle Acetylacetonate, unabhängig vom Zentralmetall nahezu gleich sein.

Neben den bereits qualitativ untersuchten Metallsalzen sollten weitere Metalle und Metallsalze einbezogen und hinsichtlich ihrer quantitativen Epoxidierungsaktivität untersucht werden.

Im folgenden Schaubild (Abb. 20) ist die Gegenüberstellung der Epoxidausbeute bei Autooxidation, mit NHPI Zusatz, mit verschiedenen Metallen/Metallsalzen und mit den binären katalytischen Systemen aufgeführt. In der Legende ist zusätzlich die Selektivität der Epoxidbildung entsprechend der angeführten Definition in eckigen Klammern wiedergegeben. 


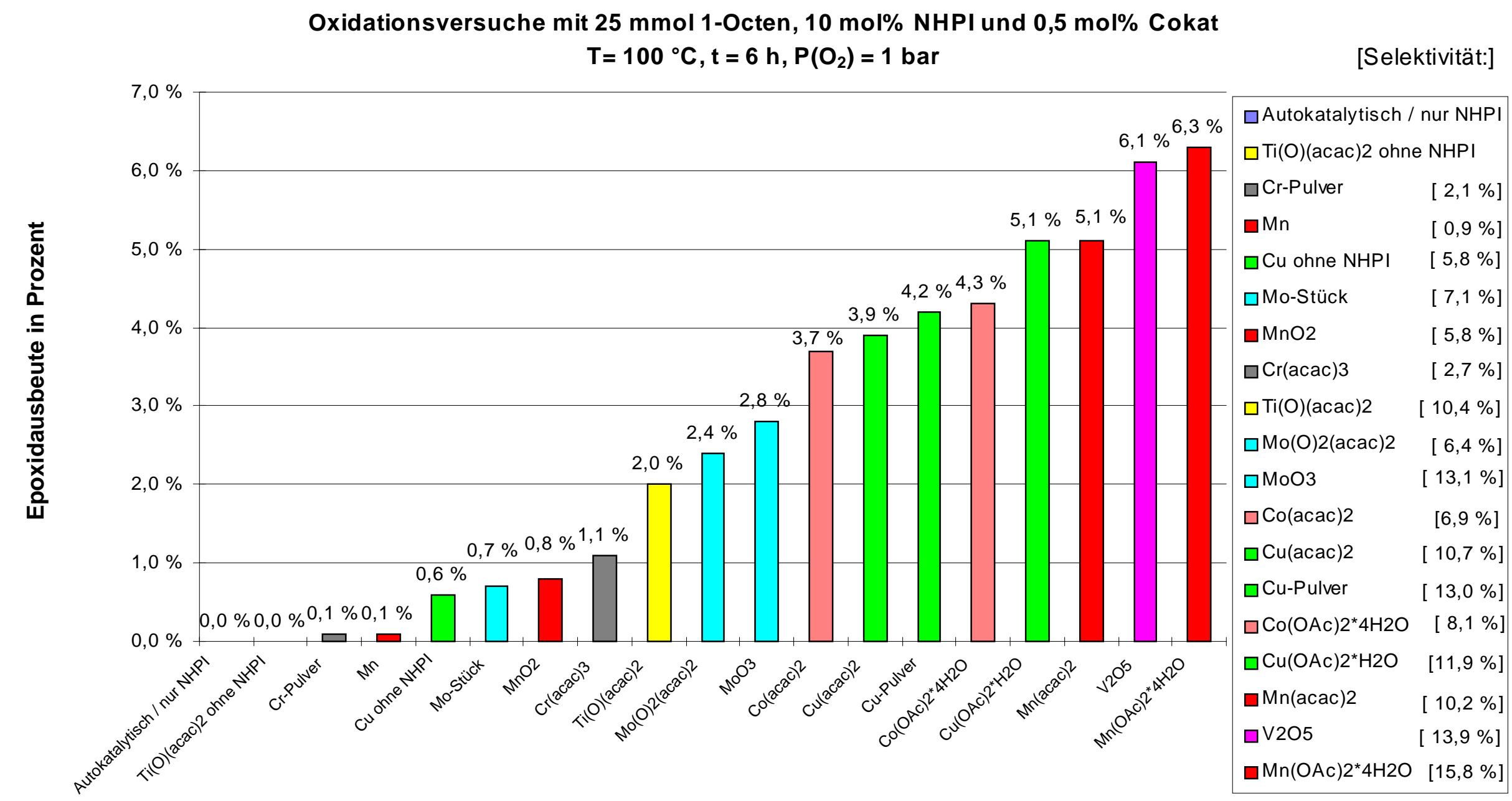

metallischer Katalysator

Abb. 8 
Das System Mangan-(II)-acetat-Tetrahydrat mit NHPI ergab unter den Reaktionsbedingungen die höchste Epoxidausbeute (Abb. 20). Deshalb wurde mit diesem System eine Zeitreihe erstellt (Abb. 21).

Da der interne Standard nach der Reaktion zugegeben werden mußte, war eine Probenentnahme während der Reaktionen nicht möglich. Die Reaktionen wurde entsprechend in den acht Reaktoren der Testapparatur gleichzeitig gestartet und zu unterschiedlichen Zeiten abgebrochen. 


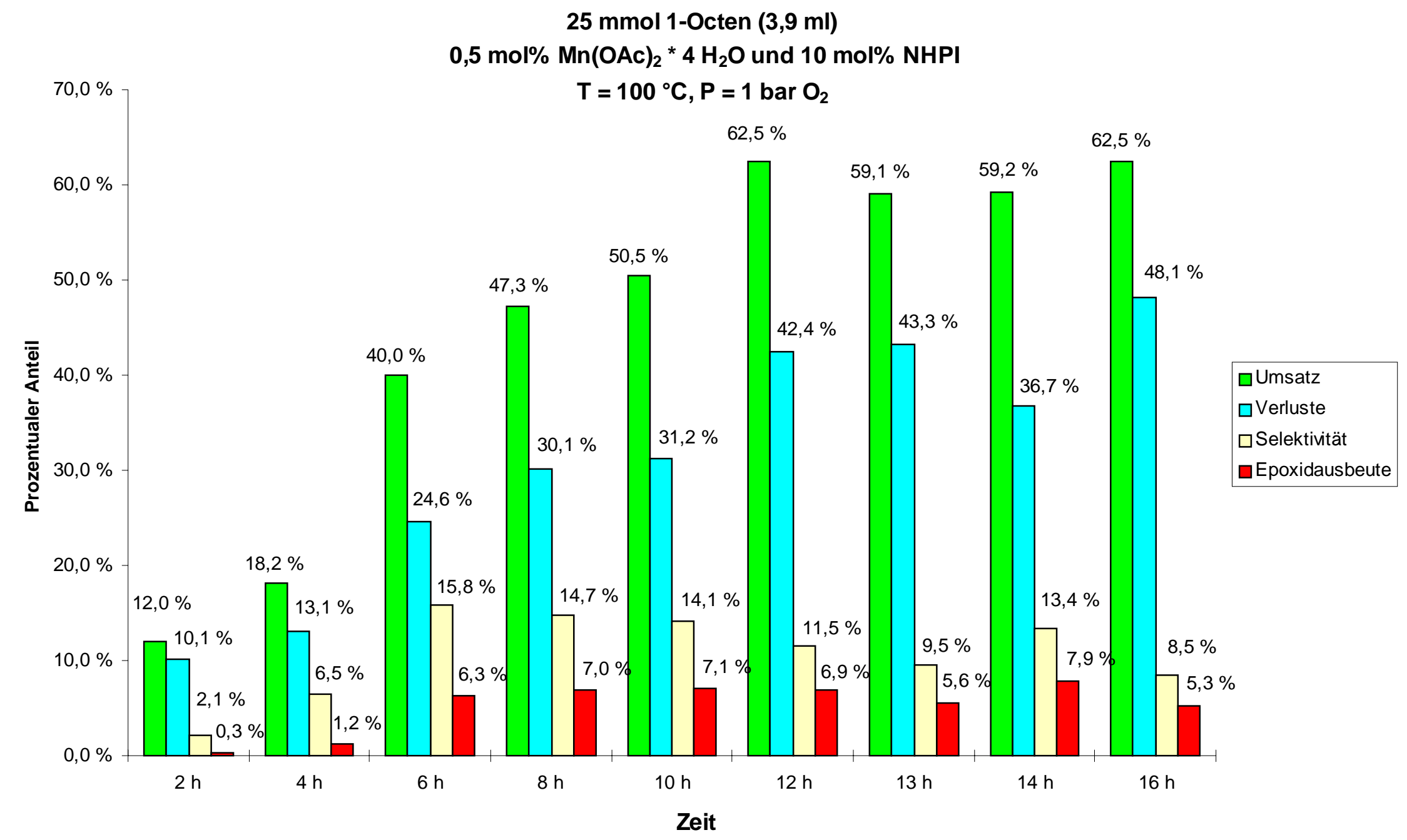

Abb. 9 
Es zeigt sich, daß für das betrachtete System (Abb. 21) eine Reaktionsdauer von sechs Stunden hinsichtlich der Epoxidausbeute und der Selektivität der Reaktion ein Optimum bildet. Bei längeren Reaktionszeiten steigt zwar der Umsatz an Octen, aber die Bildung von Nebenprodukten und Verlusten übersteigt die Epoxidbildung, so daß die Selektivität der Reaktion abnimmt. Dieser empirische Befund läßt darauf schließen, daß die Bildung der Epoxidfolgeprodukte (siehe S. 18) mit der Zeit dominant wird.

Im folgenden sollte deshalb untersucht werden, ob die Epoxidausbeute bei einer höheren Reaktionstemperatur schon nach vier Stunden zu erreichen ist.

Abbildung 22 zeigt jedoch, daß die Epoxidausbeute bei einer um 10 Kelvin erhöhten Reaktionstemperatur entscheidend zurückging. Diese Beobachtung wird darauf zurückgeführt, daß bei dieser Temperatur der Dampfdruck der Reaktionslösung stark steigt und somit der Partialdruck des Sauerstoffs sinkt. Die von der Sauerstoffzufuhr unabhängigen Nebenreaktionen werden hingegen kaum behindert, so daß zudem die Selektivität abnimmt. 
Oxidationsversuche mit $25 \mathrm{mmol} 1-$ Octen, $10 \mathrm{~mol} \% \mathrm{NHPI}$ und 0,5 mol\% Kat $\mathrm{T}=110^{\circ} \mathrm{C}, \mathrm{t}=4 / 6 \mathrm{~h}, \mathrm{P}\left(\mathrm{O}_{2}\right)=1 \mathrm{bar}$

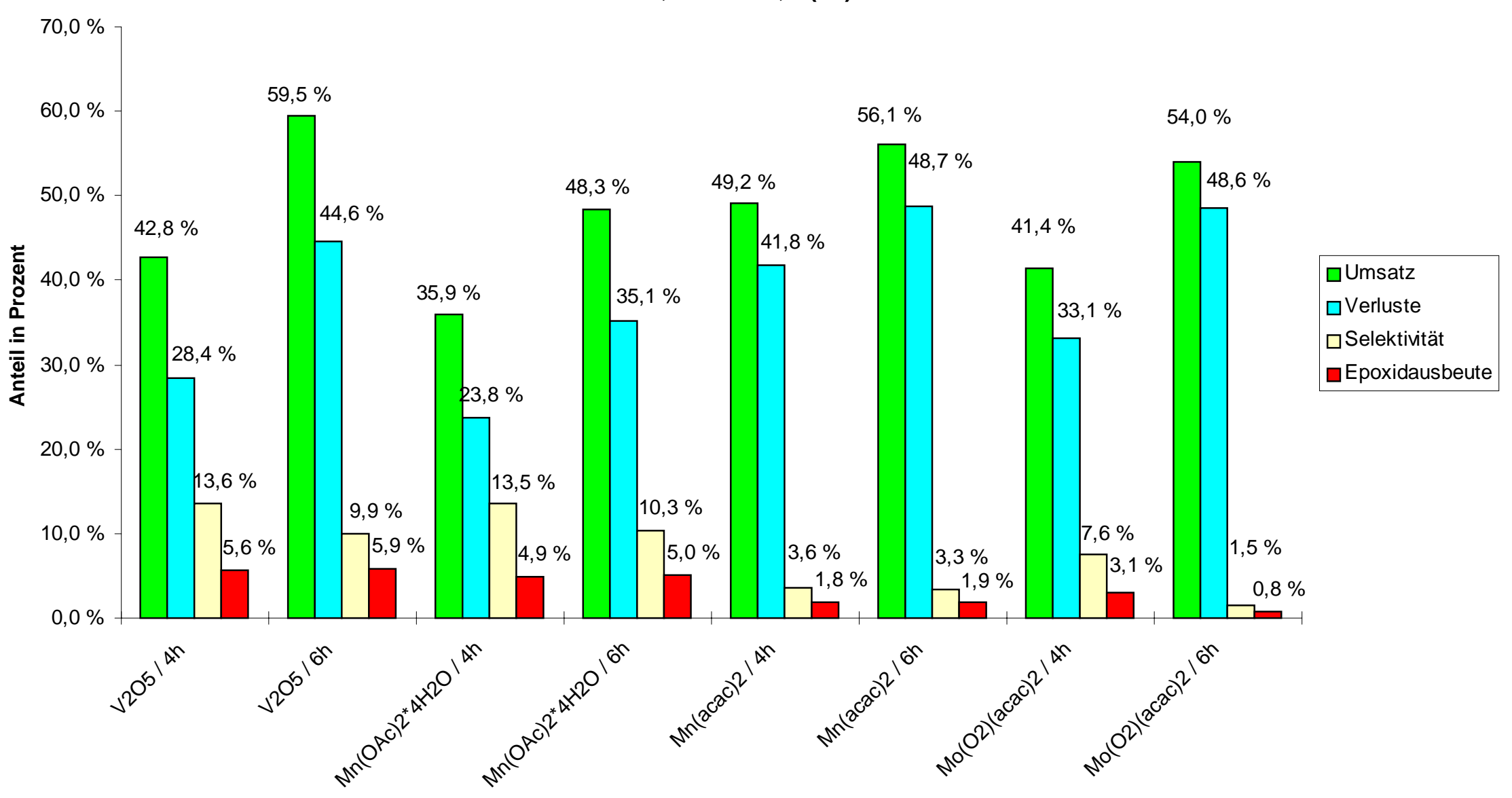

Kat. / Zeit

Abb. 10 
Aus den Vorversuchen ergab sich die Frage, ob die katalytische Aktivität der reinen Metallsalze wirklich grundsätzlich niedriger ist, als in Kombination mit NHPI. Nachdem die optimale Reaktionszeit (bei Zusatz von 10 mol\% NHPI und 0.5 mol\% Metall/Metallsalz als Cokatalysator) empirisch mit sechs Stunden bestimmt wurde (Abb. 21), untersuchte man den Einfluß der NHPI Konzentration auf die Epoxidbildung und Selektivität (Abb. 23 und 24). 


\section{$\mathrm{Mn}(\mathrm{OAc})_{2}{ }^{*} \mathbf{4} \mathrm{H}_{2} \mathrm{O} /$ NHPI System}

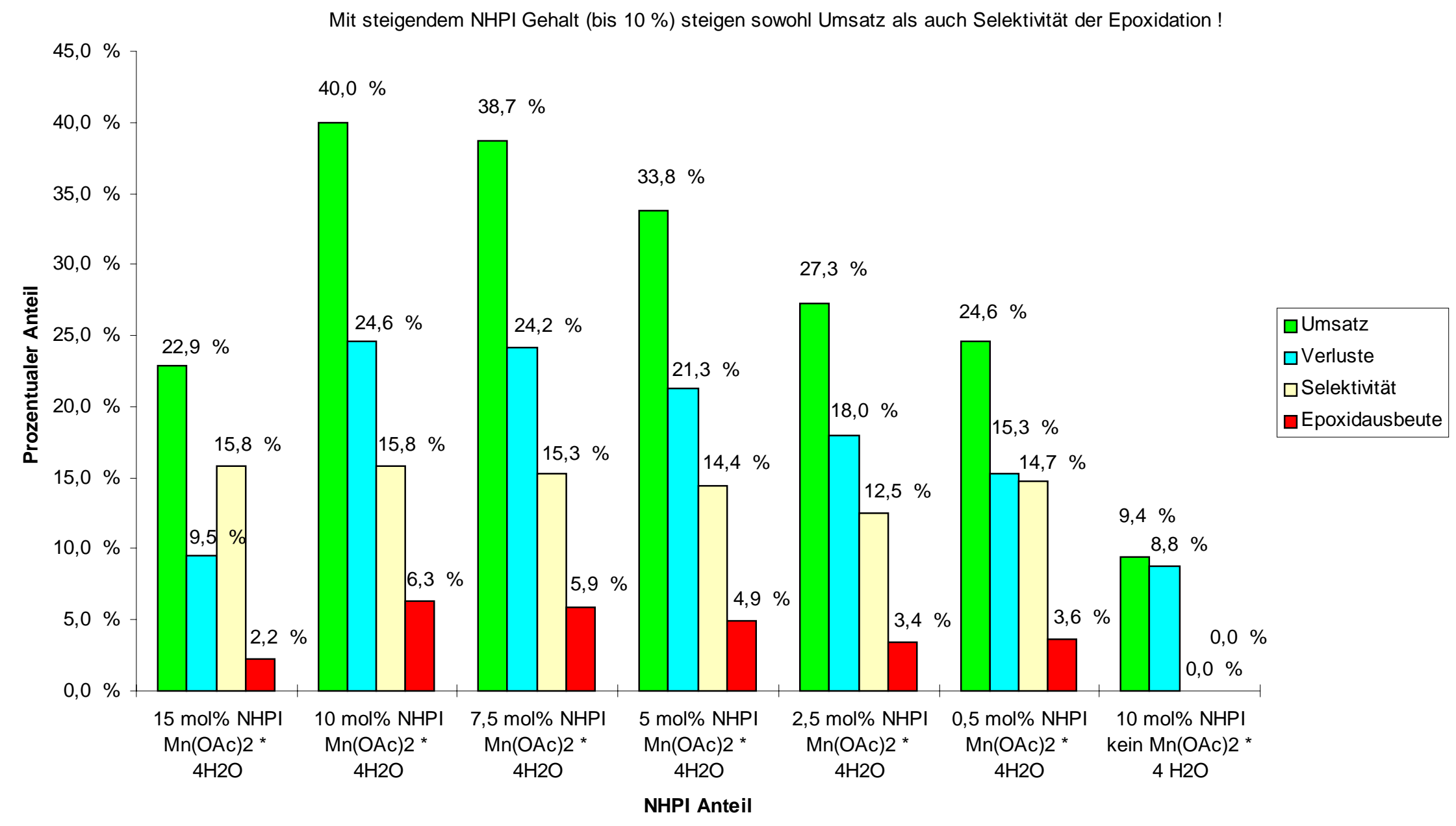

Abb. 11 


\section{$\mathrm{Cu}(\mathrm{OAc})_{2}{ }^{*} \mathrm{H}_{\mathbf{2}} \mathrm{O} /$ NHPI System}

Mit steigendem NHPI Gehalt (bis 10 mol \%) steigen sowohl Umsatz als auch Selektivität der Epoxidation!

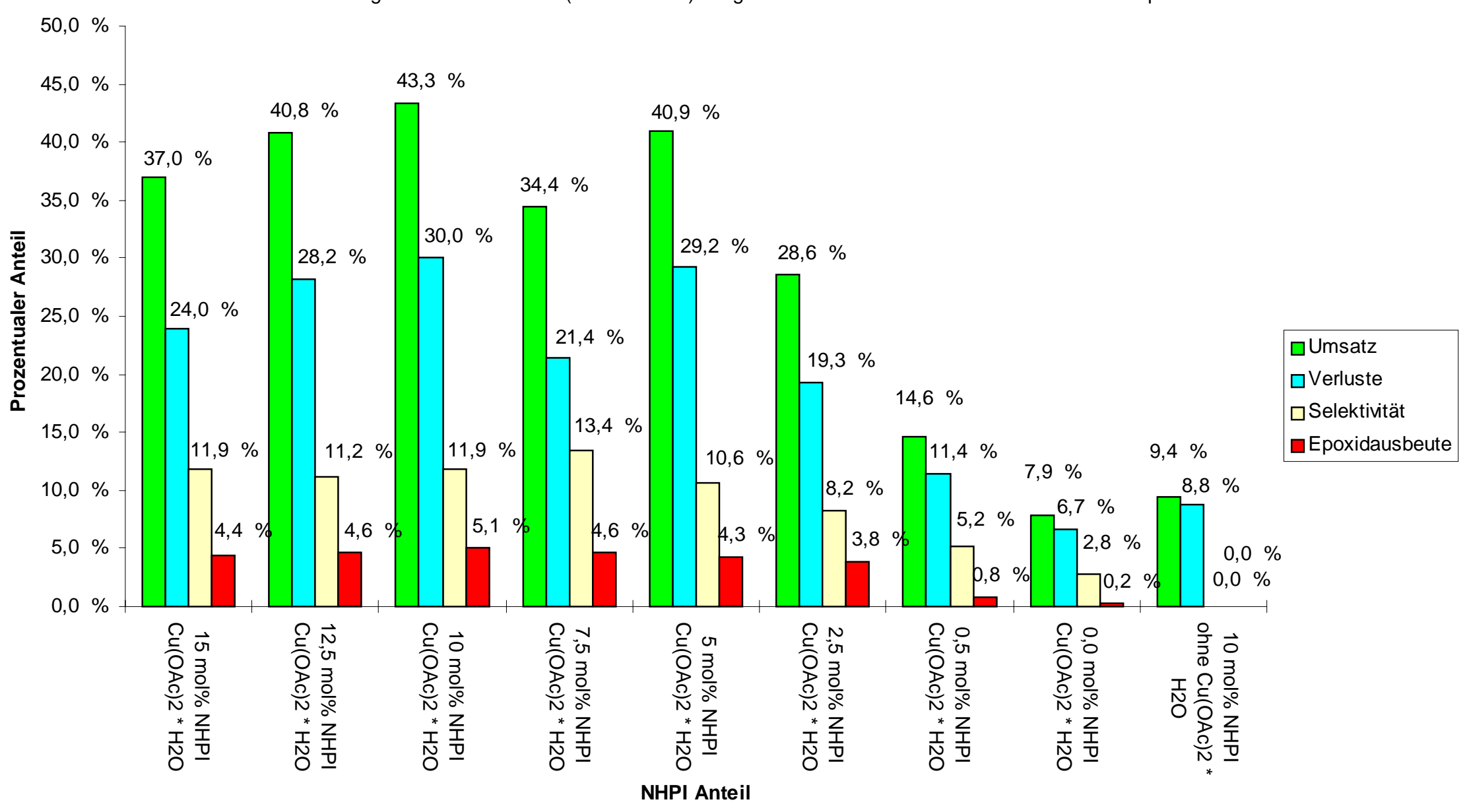

Abb. 12 
NHPI alleine bewirkte in keinem der untersuchten Fälle (Abb. 23 und 24) eine Epoxidbildung. In beiden Fällen ist jedoch zu beobachten, daß die Kombination der Metallsalze mit NHPI zu einer deutlichen Steigerung der Epoxidbildung und auch der Selektivität führt, bis bei ca. 10 mol\% ein Maximum erreicht wird. Da sich die Menge des eingesetzten 1-Octens und des Metallsalzanteils nicht geändert hat, ist davon auszugehen, daß das Verhältnis von Metallsalz zu NHPI mit $0.5 \mathrm{~mol} \%$ zu $10 \mathrm{~mol} \%$ optimiert ist.

Es sei an dieser Stelle darauf hingewiesen, daß nur die Daten der ca. 130 GCMessungen, die zur Erstellung der Abbildungen verwendet wurden, abgedruckt und besprochen werden konnten. Der Rest der Daten aus insgesamt mehr als 300 GC-Messungen konnte aufgrund der Fülle in dieser Arbeit nicht aufgeführt werden. 


\section{Bewertung der empirischen Daten}

Aus der Untersuchung der binären Katalysatorsysteme aus Metall/Metallsalz und NHPI geht hervor, daß diese Systeme zur Epoxidation von 1-Octen grundsätzlich geeignet sind. Aufgrund der hohen Verluste durch Bildung von Epoxidfolgeprodukten sind die Epoxidausbeuten und die Selektivitäten der Reaktionen mit 1-Octen unter den geschilderten Bedingungen beschränkt.

Die weitere wissenschaftliche Erforschung zur Verbesserung der Selektivität oder der direkten Epoxidausbeute ist daher dringend erforderlich, um eine Anwendung der katalytischen Systeme zur gezielten Epoxiddarstellung zu ermöglichen.

\subsection{Beurteilung der Epoxidausbeute}

Wenn man den hohen Anteil an nicht GC-flüchtigen Nebenprodukten (Verluste) bei der Auswertung vernachlässigt oder sie als Epoxidfolgeprodukte der Epoxidausbeute zurechnet, erhält man Ausbeuten größer als $50 \%$ mit einer guten Selektivität. Obwohl diese Vorgehensweise theoretisch zu rechtfertigen ist und auch angewendet wird, ist sie für die praktische Anwendung in der Epoxidierungskatalyse nicht hilfreich.

Um die in der Zielsetzung beschriebene technische Verwertbarkeit der erzielten Katalyseergebnisse zu beurteilen, dürfen nach meiner Auffassung nur isolierbare Ausbeuten an Epoxid betrachtet werden. Die in unserem Arbeitskreis verwendeten strengeren Definitionen sollten dies ermöglichen. 
Aus der Gesamtbetrachtung der durchgeführten Versuche kann geschlossen werden, daß sich die Reaktion unter anderem hinsichtlich Reaktionsdauer, Reaktionstemperatur und Sauerstoffdruck wesentlich weiter optimieren läßt.

Dabei ist ferner anzumerken, daß sich NHPI nicht in 1-Octen (und auch nicht in Cycloocten, 1-Hexen und Cyclohexen) löst. Die Katalyse mit 1-Octen als Modell-Substrat und z.B. mit Cu-Pulver als Katalysatorkomponente ist also größtenteils heterogen. (Eventuell steht nur der gelöste Bruchteil an NHPI für die Reaktion zur Verfügung ?!).

Bei der Reaktionsdurchführung unter hohem Druck mit Propen als Substrat könnte die Löslichkeit von NHPI größer sein. Die Radikalreaktion wird dabei gefördert. Zudem sollten die Verluste bei Propen geringer und somit die Selektivität höher sein, weil die meisten Zwischenstufen, die zu den Nebenprodukten führten, bei Propen weniger stabil sind als bei 1-Octen. Unter erhöhtem Druck kann zudem die Reaktionstemperatur gesteigert werden, ohne den Sauerstoffpartialdruck über der Reaktionslösung zu senken. (Bisher mußte die Reaktion ca. $20{ }^{\circ} \mathrm{C}$ unter dem Siedepunkt der Lösung durchgeführt werden, um dem Sauerstoff den Zugang zur Reaktionslösung zu ermöglichen.) Der Einsatz von Ultraschall sollte die Radikalreaktion durch SET (Single Electron Transfer) ebenfalls günstig beeinflussen. 


\subsection{Modell zum Reaktionsmechanismus}

Die Ergebnisse der Katalysetests, insbesondere der Vergleich der verschiedenen Acetylacetonate zeigen, daß die Epoxidation nicht nur über das Ligandensystem des eingesetzten Metalls verlaufen kann. Im Falle des Kupfers ist sogar ohne Ligandensphäre eine Epoxidationsaktivität feststellbar. Da NHPI unter den Reaktionsbedingungen alleine keine nennenswerte Epoxidationsaktivität aufweist, muß ein Zusammenspiel von Metallzentrum und NHPI die Epoxidation des Substrates bewirken.

Der Nachweis eines Reaktionsmechanismus der NHPI gestützten Katalyse durch Identifizierung oder gar Isolierung der reaktiven Spezies ist bisher nicht erfolgt.

Keiner der „klassischen“ Reaktionsmechanismen über:

- Metallhydroperoxide

$-2+2$ Cycloaddition

- 1,3 Dipolmechanismus

- Peroxosäuren und Alkylhydroperoxide

- Autooxidation über gebildete Alkylhydroperoxide gibt einen direkten Hinweis auf die Förderung der Epoxidation durch NHPI.

Der Vergleich mit NHPI gestützten Katalysesystemen, wie sie in der Literatur beschrieben werden, gestaltet sich schwierig, da sich die Reaktionsbedingungen und Substrate unterscheiden. 
Masui et al. setzten in der NHPI katalysierten Reaktion Tetraphenylporphyrinato-Mangan-(III)-chlorid als Metallsalz oder metallorganische Komponenten ein, um biologische Cytochromsysteme zu imitieren. Als Substrat wurden aktivierte Alkene wie Styren oder Cyclohexen verwendet. Mit 1-Octen sind die Epoxidausbeuten aber sehr gering. Die Reaktionen finden in Acetonitril als Lösungsmittel statt, dem zusätzlich Pyridin zugesetzt werden muß, um die Katalyse zu ermöglichen.

Als Reaktionsmechanismus wird ein vielstufiger, radikalischer Verlauf über Pyridin-Sauerstoff und NHPI-Sauerstoff Addukte diskutiert. Dabei soll ein Sauerstoffatom auf das Metallzentrum übertragen werden. Das Metallzentrum epoxidiert dann das Alken und durchläuft so einen Oxidationsstufenwechsel um zwei Einheiten. ${ }^{[25]}$

Auch nach Ishii et al. ist ein durch NHPI Autooxidation gebildetes Phthalimid-NOxyl-Radikal an der Reaktion beteiligt. Dies konnte durch ESR Untersuchungen nachgewiesen werden. Das Radikal entreißt dem Substrat ein Wasserstoffatom und überträgt somit die Radikaleigenschaft. Das Substratradikal kann dann mit dem molekularen Sauerstoff $\mathrm{zu}$ den Produkten reagieren. Die Rolle des Metallsalzes war dabei nicht aufzuklären, wird jedoch in der Unterstützung der Bildung des Phthalimid-N-Oxyl-Radikals vermutet. ${ }^{[17]}$

$\mathrm{Da}$ in unseren Systemen die Katalyse ohne Zugabe eines Metallsalzes oder Metalls keine Epoxidierungsaktivität zeigt, muß ein radikalischer Verlauf über Metallperoxide vermutet werden, der dem von Masui et al. beschriebenen Reaktionsverlauf ähnlich ist. Hierbei sind dann bereits beschriebene Mechanismen für die Epoxidierung mit Metallsalzen zu berücksichtigen. 


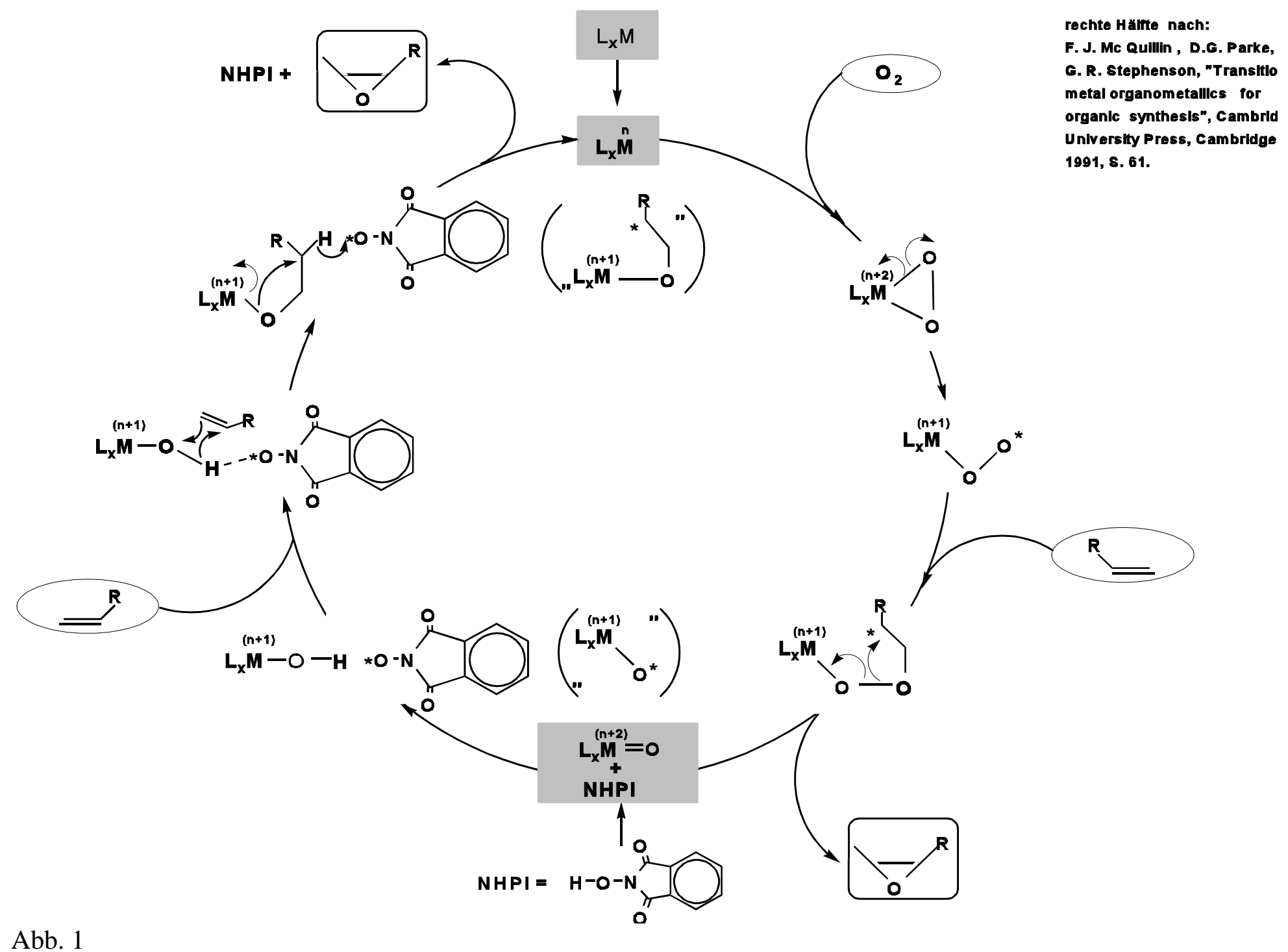


Nach dem ersten Schritt des Reaktionszyklus (Abb. 25) auf der rechten Seite ist zusätzlich eine durch NHPI verursachte Ringöffnung zum Metallhydroperoxid denkbar. NHPI könnte zudem vor dem dritten Schritt das Alken in ein Alkylradikal umwandeln und somit die Addition an das Metallperoxid oder Hydroperoxid erleichtern.

Zur Plausibilität der abgebildeten Arbeitshypothese tragen die folgenden Erkenntnisse bei:

- Die größte katalytische Aktivität zeigten Metalle und Metallsalze mit drei benachbarten, bevorzugten Oxidationsstufen wie Mn (II,III,IV), V (III,IV,V), $\mathrm{Cu}(0, \mathrm{I}, \mathrm{II})$ und Mo (II,III,IV).

(Die Ausnahme bildet Cobalt, bei dem der Sauerstoff im NHPI Zyklus vermutlich das Metallzentrum nicht oxidiert, sondern von diesem nur koordiniert wird. Eventuell ist im Falle des Cobalts auch der von Ishii et al. postulierte Mechanismus dominant.)

- Die Ausbildung von Metallperoxiden und Superoxiden mit Wasserstoffperoxid ist für die Metalle Co, $\mathrm{Rh}, \mathrm{Ir}, \mathrm{Cr}, \mathrm{Mo}, \mathrm{W}, \mathrm{V}, \mathrm{Mn}, \mathrm{Cu}$ und andere (Ti, Fe, Ni, Pd, Pt usw.) bekannt. ${ }^{[2]}$ (Mit molekularem Sauerstoff bilden allerdings nur einige Metalle wie Mangan, Platin, Kupfer und Titan Peroxide.)

- Die guten Ergebnisse hinsichtlich Selektivität und Ausbeute bei der Verwendung von Vanadiumoxid aber auch Kupferpulver (!) lassen sich mit dem oben entworfenen Zyklus vereinbaren. 
- Die Ausbildung Hydroxylgruppen-haltiger Nebenprodukte durch Übertragung eines Wasserstoffatoms auf das Alkyl-Sauerstoffradikal im dritten Reaktionsschritt (Abb. 25) ist plausibel.

- Mit dem entworfenen Katalysezyklus läßt sich der erforderliche NHPI Zusatz (zur Metallsalz-Komponente) erklären. Ohne NHPI endet die Katalyse mit der Ausbildung des Metalloxids.

- Eventuell wird das NHPI Radikal in der Reaktion von radikalischen Oxidationszwischenprodukten abgefangen und desaktiviert.

Die Ausbildung derartiger Nebenprodukte könnte zum beobachteten Verlust bei der gaschromatographischen Analyse beigetragen haben. 


\subsection{Zusammenfassung}

Das in der Aufgabenstellung benannte Ziel konnte erreicht werden. Es ist ein neues katalytisches Verfahren entwickelt worden, das die Epoxidation von nichtaktivierten Alkenen mit molekularem Sauerstoff ermöglicht. Darüber hinaus konnte ein geschlossener Katalysezyklus für die katalytische Epoxidation bei Normaldruck postuliert werden, der mit allen bisher gefundenen Daten und publizierten Ergebnissen schlüssig ist. Dabei werden zudem die prinzipiellen Vorteile der Epoxidation mit molekularem Sauerstoff verdeutlicht.

Wenn der postulierte Mechanismus richtig ist, werden beide Sauerstoffatome bei der Oxidation umgesetzt und für die Epoxidation verwendet. Demnach wird kein Wasser gebildet. Da auch keine Lösungsmittel erforderlich sind entfallen zwei Trennprobleme. Die Reaktion verläuft zudem halogenfrei, was aus Gründen des Umweltschutzes aber auch von technischer Seite hinsichtlich der Korrosionsproblematik vorteilhaft ist. 


\section{Präparative Projekte}

\subsection{Titanophosphonate}

Analog zu den eingangs beschriebenen Titanosilicaten wie TS1 und Zeolithen sollten auch strukturähnliche Titanophosphonate zur Epoxidation geeignet sein. Neben den Versuchen zur Epoxidationskatalyse mit den beschriebenen binären Systemen wurde deshalb die Synthese von molekularen, in organischen Lösungsmitteln löslichen Titanophosphonaten vorangetrieben.

Die direkte Umsetzung von $\mathrm{Cp} * \mathrm{TiCl}_{3}, \mathrm{Cp}^{*} \mathrm{TiMe}_{3}$ und $\mathrm{Cp} * \mathrm{ZrCl}_{3}$ mit kristalliner Phosphorsäure unter verschiedenen Bedingungen führte jedoch $\mathrm{zu}$ keinem Ergebnis. Es wurden deshalb unterschiedlich substituierte Phosphonsäuren eingesetzt. Durch Reaktion von $\mathrm{Cp}^{*} \mathrm{TiMe}_{3}$ mit $\mathrm{RPO}(\mathrm{OH})_{2}(\mathrm{R}=\mathrm{Ph}, \mathrm{Me})$ konnten im Arbeitskreis kubische Titanophosphonate in guter Ausbeute dargestellt werden, die in den meisten gebräuchlichen Lösungsmitteln löslich sind. ${ }^{[27]}$ Bei der zeitgleich betriebenen Umsetzung von $\mathrm{Cp}^{*} \mathrm{TiMe}_{3}$ mit tert-Butylphosphonsäure erhielt ich jedoch keine Verbindung mit analoger Zusammensetzung. Aus dem Massenspektrum des nach dem Entfernen des Lösungsmittels zurückgebliebenen Rückstandes war zu erkennen, daß dem Reaktionsprodukt je eine Einheit Phosphonsäure und ein Fragment Cp*-Titan fehlen. Im ${ }^{31}$ P NMR- Spektrum zeigten sich drei große und 5 kleinere Signale. Durch Kristallisation des Rückstandes konnten Einkristalle 1 erzeugt werden:

$$
\begin{gathered}
3 t-\mathrm{BuP}(\mathrm{O})(\mathrm{OH})_{2}+3 \mathrm{Cp}^{*} \mathrm{TiMe}_{3}+2 \mathrm{H}_{2} \mathrm{O} \rightarrow \\
{\left[(\mathrm{Cp} * \mathrm{Ti})_{3}\left(t-\mathrm{BuPO}_{3}\right)_{2}\left\{t-\mathrm{BuPO}_{2}(\mathrm{OH})\right\}(\mu-\mathrm{O})_{2}\right](\mathbf{1})}
\end{gathered}
$$

Abb. 26 
Struktur von 1 im Kristall:

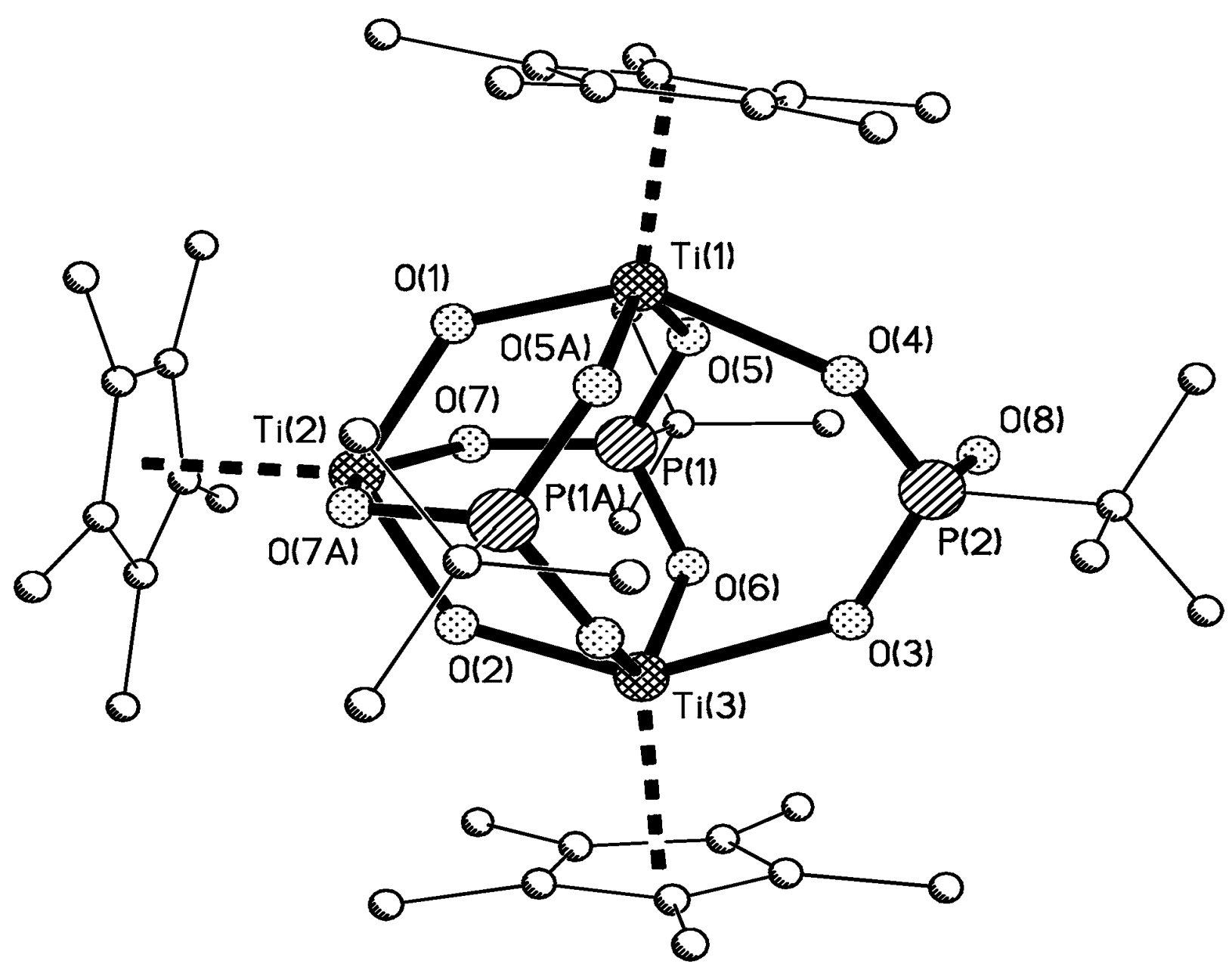

Abb. 2 
Die kristallographische Untersuchung zeigt eine korbähnliche Struktur der Verbindung 1 (Abb. 27).

Aus der Abbildung geht hervor, daß jedes Phosphoratom $\left(\mathrm{P}_{1}, \mathrm{P}_{1(\mathrm{~A})}, \mathrm{P}_{2}\right)$ eine unterschiedliche chemische Umgebung hat und somit drei Signale im ${ }^{31} \mathrm{P}$ NMR Spektrum zu erwarten sind. Man ordnete Verbindung 1 deshalb drei dominierenden Signale gleicher Intensität im ${ }^{31}$ P NMR Spektrum der Kristalle zu. Den beiden dreifach verbrückenden $t-\mathrm{BuPO}_{3}$ Einheiten $\left(\mathrm{P}_{1}, \mathrm{P}_{1 \mathrm{~A}}\right)$ wurden dabei zwei benachbarte Signale ( $\delta 35.9 \mathrm{ppm}$ und $35.6 \mathrm{ppm}$ ) zugeordnet. Der $t$ - $\mathrm{BuPO}_{2}(\mathrm{OH})$ Gruppe $\left(\mathrm{P}_{2}\right)$ ordnete man das dritte Signal bei $\delta 31.9 \mathrm{ppm} z u$. Die Herkunft der anderen fünf Signale, die auch im ${ }^{31} \mathrm{P}$ NMR Spektrum der Kristalle noch identifizierbar waren, konnte zu diesem Zeitpunkt nicht geklärt werden, da kein weiteres Produkt aus der Kristallisationslauge isoliert werden konnte.

Um die Darstellung der Titanophosphonate zu vereinfachen, wurde die Reaktion von $\mathrm{Cp}^{*} \mathrm{TiCl}_{3}$ mit substituierten Phosphonsäuren untersucht. Durch Zugabe von Triethylamin als Base und mit einem Überschuß der Phosphonsäurekomponente konnten die kubischen Produkte ebenfalls dargestellt werden. ${ }^{[27]}$

Bei der Reaktion von äquimolaren Mengen oder einem Überschuß an tertButylphosphonsäure mit $\mathrm{Cp}^{*} \mathrm{TiCl}_{3}$ unter Zusatz von Triethylamin erhielt ich jedoch wiederum als Hauptprodukt Verbindung 1. Bei der Identifizierung mit Hilfe des ${ }^{31} \mathrm{P}$ NMR Spektrums zeigten sich erneut viele Signale, die der Verbindung 1 nicht zuzuordnen waren und im Massenspektrum fand man den Peak für das Fragment $\mathrm{M}(\mathbf{1})^{+}-\mathrm{Cp}^{*}$. 
Setzt man $\mathrm{Cp}^{*} \mathrm{TiCl}_{3}$ im Überschuß ein, erhält man eine tiefviolette Verbindung mit einem Massenspektrum, das wiederum dem der Verbindung 1 ähnlich ist. Im ${ }^{31}$ P NMR Spektrum fehlen jetzt jedoch die Signale von Verbindung 1 völlig und ein Teil der fünf vorher nicht identifizierbaren Signale wird dominant.

Durch Rekristallisation des getrockneten Rückstandes aus Trichlormethan konnten Einkristalle erhalten und strukturanalytisch aufgeklärt werden (Abb. 28, Verbindung 2). Die kristallographisch ermittelte Zusammensetzung von Verbindung $2\left(\mathrm{C}_{28} \mathrm{H}_{51} \mathrm{Cl}_{2} \mathrm{O}_{7} \mathrm{P}_{2} \mathrm{Ti}_{2}\right)$ konnte durch eine Elementaranalyse bestätigt werden.

Eine spektroskopische Bestätigung von Verbindung 2 nach dem Entfernen des Lösungsmittels im Vakuum war hingegen nicht möglich, da sich der zurückgebliebene Feststoff nicht wieder vollständig in Trichlormethan oder Benzol löste. Man erhielt eine dunkelorange Lösung mit gelben und leuchtend roten Feststoffpartikeln. Die NMR Spektren der Lösung sowie das Massenspektrum des Feststoffes ähneln dabei denen von Verbindung 1.

Es darf somit vermutet werden, daß 2 eine metastabile Vorstufe der Reaktion zu Verbindung 1 darstellt. Bei dem vollständigen Entfernen des Lösungsmittels sowie beim Aufheizen der Probe zur massenspektroskopischen Untersuchung zersetzt sich Verbindung 2 unter Bildung von Verbindung 1 und weiterer Produkte.

Da sich die Bindungsverhältnisse der Sauerstoffatome sowie die Positionen der Wasserstoffatome aus den gefundenen Daten nicht eindeutig bestimmen lassen, stellt Abbildung 28 nur eines der möglichen Bindungsverhältnisse dar: 
Struktur von $\mathbf{2}$ im Kristall

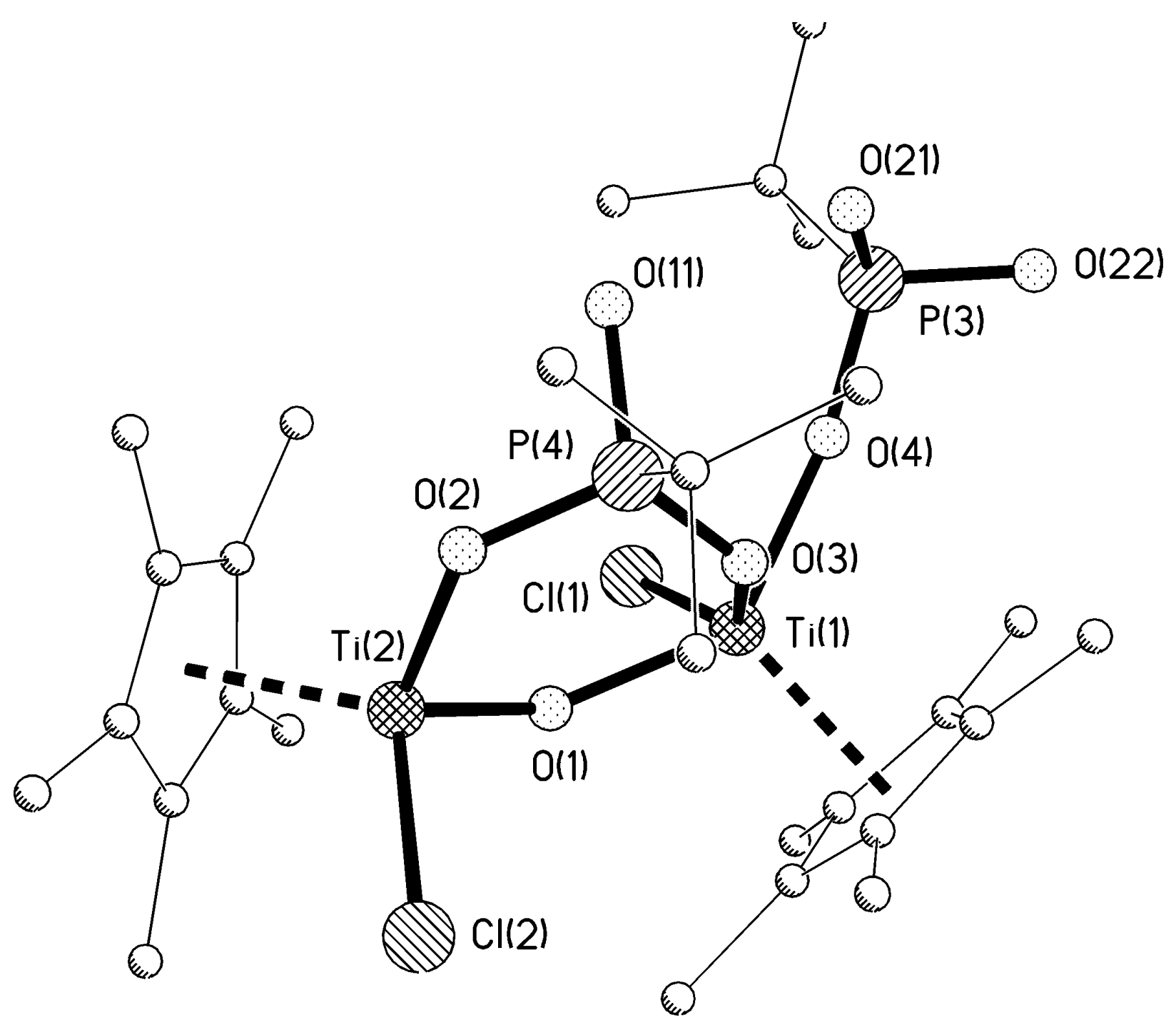

Abb. 3 
Vergleich ausgesuchter Bindungslängen ( $\mathrm{pm}$ ) in Verbindung 1 und 2:

\begin{tabular}{|c|c|c|c|}
\hline Verbindung 1 & Verbindung 2 & Verbindung 1 & Verbindung 2 \\
\hline \multicolumn{2}{|c|}{ Ti-O Abstände } & \multicolumn{2}{|c|}{ P-O Abstände } \\
\hline $\mathrm{Ti}_{1}-\mathrm{O}_{1}\left(-\mathrm{Ti}_{2}\right) 179.9$ & $\mathrm{Ti}_{1}-\mathrm{O}_{1}\left(-\mathrm{Ti}_{2}\right) 191.6$ & $\mathrm{P}_{1}-\mathrm{O}_{5}\left(-\mathrm{Ti}_{1}\right) 154.1$ & $\mathrm{P}_{3}-\mathrm{O}_{4}\left(-\mathrm{Ti}_{1}\right) 151.7$ \\
\hline $\mathrm{Ti}_{2}-\mathrm{O}_{1}\left(-\mathrm{Ti}_{1}\right) 187.5$ & $\mathrm{Ti}_{2}-\mathrm{O}_{1}\left(-\mathrm{Ti}_{1}\right) 175.3$ & $\mathrm{P}_{1}-\mathrm{O}_{6}\left(-\mathrm{Ti}_{3}\right) 154.0$ & $\mathrm{P}_{4}-\mathrm{O}_{2}\left(-\mathrm{Ti}_{2}\right) 153.9$ \\
\hline $\mathrm{Ti}_{2}-\mathrm{O}_{2}\left(-\mathrm{Ti}_{3}\right) 184.2$ & & $\mathrm{P}_{1}-\mathrm{O}_{7}\left(-\mathrm{Ti}_{2}\right) 152.9$ & $\mathrm{P}_{4}-\mathrm{O}_{3}\left(-\mathrm{Ti}_{1}\right) 151.4$ \\
\hline $\mathrm{Ti}_{3}-\mathrm{O}_{2}\left(-\mathrm{Ti}_{2}\right) 182.5$ & & $\mathrm{P}_{2}-\mathrm{O}_{3}\left(-\mathrm{Ti}_{3}\right) 153.8$ & \\
\hline $\mathrm{Ti}_{1}-\mathrm{O}_{4}\left(-\mathrm{P}_{2}\right) 208.4$ & $\mathrm{Ti}_{1}-\mathrm{O}_{3}\left(-\mathrm{P}_{4}\right) 206.8$ & $\mathrm{P}_{2}-\mathrm{O}_{4}\left(-\mathrm{Ti}_{1}\right) 151.3$ & \\
\hline $\mathrm{Ti}_{1}-\mathrm{O}_{5}\left(-\mathrm{P}_{1}\right) 203.6$ & $\mathrm{Ti}_{1}-\mathrm{O}_{4}\left(-\mathrm{P}_{3}\right) 197.7$ & $\mathrm{P}_{2}-\mathrm{O}_{8}(-\mathrm{H}) 158.9$ & $\mathrm{P}_{3}-\mathrm{O}_{22}(-\mathrm{H}) 156.5$ \\
\hline $\mathrm{Ti}_{2}-\mathrm{O}_{7}\left(-\mathrm{P}_{1}\right) 207.4$ & $\mathrm{Ti}_{2}-\mathrm{O}_{2}\left(-\mathrm{P}_{4}\right) 191.2$ & & $\mathrm{P}_{3}-\mathrm{O}_{21}(-\mathrm{H}) 151.6$ \\
\hline $\mathrm{Ti}_{3}-\mathrm{O}_{6}\left(-\mathrm{P}_{1}\right) 201.0$ & & & $\mathrm{P}_{4}-\mathrm{O}_{11}(-\mathrm{H}) 155.0$ \\
\hline $\mathrm{Ti}_{3}-\mathrm{O}_{3}\left(-\mathrm{P}_{2}\right) 206.7$ & & & \\
\hline
\end{tabular}

Vergleich ausgesuchter Bindungswinkel $\left({ }^{\circ}\right)$ in Verbindung 1 und 2:

\begin{tabular}{|c|c|c|c|}
\hline \multicolumn{2}{|c|}{ Verbindung 1} & \multicolumn{2}{c|}{ Verbindung 2} \\
\hline \hline $\mathrm{Ti}_{1}-\mathrm{O}_{1}-\mathrm{Ti}_{2}$ & 134.44 & $\mathrm{Ti}_{1}-\mathrm{O}_{1}-\mathrm{Ti}_{2}$ & 146.2 \\
\hline $\mathrm{Ti}_{2}-\mathrm{O}_{2}-\mathrm{Ti}_{3}$ & 136.14 & & \\
\hline \hline $\mathrm{Ti}_{1}-\mathrm{O}_{4}-\mathrm{P}_{2}$ & 141.40 & $\mathrm{Ti}_{1}-\mathrm{O}_{3}-\mathrm{P}_{4}$ & 132.5 \\
\hline $\mathrm{Ti}_{1}-\mathrm{O}_{5}-\mathrm{P}_{1}$ & 125.91 & $\mathrm{Ti}_{1}-\mathrm{O}_{4}-\mathrm{P}_{3}$ & 161.5 \\
\hline $\mathrm{Ti}_{2}-\mathrm{O}_{7}-\mathrm{P}_{1}$ & 119.16 & $\mathrm{Ti}_{2}-\mathrm{O}_{2}-\mathrm{P}_{4}$ & 128.7 \\
\hline $\mathrm{Ti}_{3}-\mathrm{O}_{6}-\mathrm{P}_{1}$ & 129.30 & & \\
\hline $\mathrm{Ti}_{3}-\mathrm{O}_{3}-\mathrm{P}_{2}$ & 133.70 & & \\
\hline
\end{tabular}


Ein Vergleich der P-O und Ti-O Bindungsabstände und Winkel von Verbindung 2 mit denen von 1 zeigt, daß weder die Titanatome noch die Phosphoratome hinsichtlich ihres Bindungsmodus äquivalent sind. Besonders auffällig in 2 ist die große Bindungslänge der $\mathrm{Ti}_{1}-\mathrm{O}_{1}$ Bindung (191.6 ppm) bei entsprechend kurzer $\mathrm{Ti}_{2}-\mathrm{O}_{1}$ Bindung (175.3 ppm). Auch der Bindungswinkel ist mit $146^{\circ}$ größer als vergleichbare Winkel in $1\left(\sim 135^{\circ}\right)$, so daß eventuell nur eine koordinative Bindung vorliegt. Bei den Phosphor-Sauerstoff-Bindungslängen findet man für die Sauerstoffatome vergleichsweise kurze Bindungsabstände, die auf eine Doppelbindung schließen lassen.

Unter Berücksichtigung der Ladungsverhältnisse, der Bindungsabstände und Winkel lassen sich die Bindungsverhältnisse von 2 entsprechend auch wie folgt darstellen:

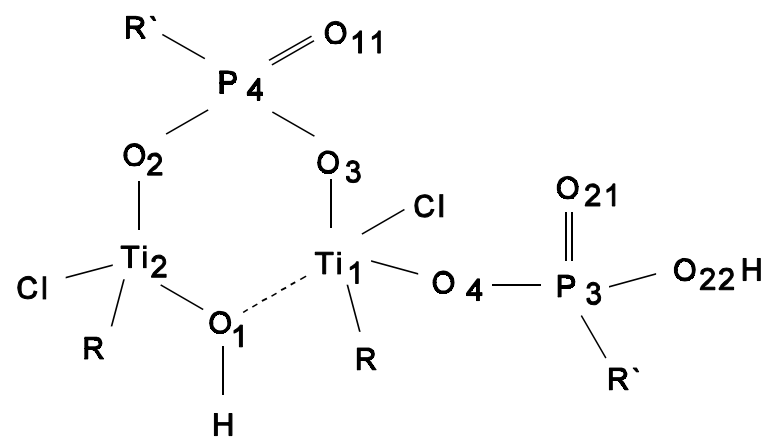

Abb. 29 


\subsection{Wasserlösliche Metallcarbene}

In einem weiteren Projekt sollte die Synthese von wasserlöslichen Metallcarbenen erforscht werden. Herrmann et al. konnten Palladiumkomplexe mit Imidazoliumliganden synthetisieren, welche eine beachtliche Aktivität in der oxidativen Heck-Reaktion aufweisen. Schon 1995 und 1997 schlugen sie die Derivatisierung zu wasserlöslichen Katalysatoren vor, um eine Zweiphasenkatalyse zu ermöglichen. ${ }^{[28]}$

Abb. 30<smiles>[R]N1C=CN([R])C1[R](CI)C1N([R])C=CN1[R]</smiles>

Versuche, das Imidazoliumsystem mit Phosphor- und Phosphon-Säuren (R) am

Stickstoff zu derivatisieren, ${ }^{[29]}$ um Wasserlöslichkeit herzustellen, schlugen fehl. In den ${ }^{31} \mathrm{P}$ NMR Spektren waren keine einheitlichen Hauptprodukte auszumachen, da unter den sauren Reaktionsbedingungen bei der Reaktion der Imidazoliumhydrohalogenide mit dem Metallacetat $\left\{2 \mathrm{RH}^{+} \mathrm{I}^{-}+\mathrm{Pd}(\mathrm{OAc})_{2} \rightarrow\right.$ $\left.\left[\mathrm{PdR}_{2}\right] \mathrm{I}_{2}+2 \mathrm{HOAc}\right\}$ die Säure- und Säureestergruppen polymerisierten oder hydrolytisch vom Stickstoff gespalten wurden und schwerlösliche Polyphosphate bildeten. ${ }^{[29 c]}$ 
Da die Stickstoff-Kohlenstoffbindung stabiler ist, als die StickstoffPhosphorbindung wurde die Reaktion analog mit Carbonsäureestern wiederholt. Die Reaktion von Methylimidazol mit Chlorameisensäureester sollte direkt zum Imidazoliumhydrochlorid führen. Nach der Umsetzung mit Palladium- oder Quecksilberacetat sollte unter Salzsäureabspaltung das entsprechend derivatisierte Carben entstehen. ${ }^{[30]}$<smiles>CC(=O)N1C=CN(C)C1C(=O)O</smiles>

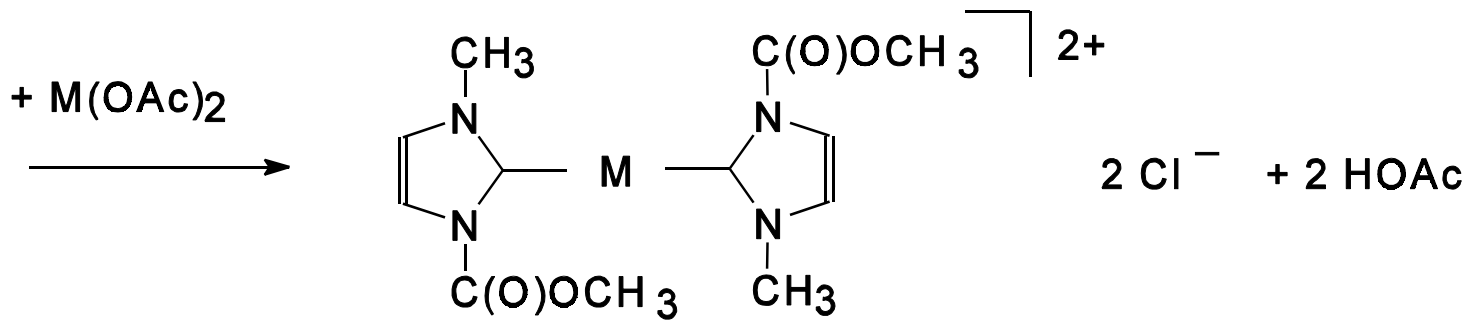

Abb. 6

Dieser Reaktionsverlauf konnte im Fall des Quecksilbers anscheinend realisiert werden und führte $\mathrm{zu}$ einer wasserlöslichen Verbindung $\mathbf{3}$, die laut Elementaranalyse dem gewünschten Produkt mit zwei zusätzlichen Molekülen Kristallwasser entsprach.

${ }^{1} \mathrm{H}$ und ${ }^{13} \mathrm{C}$ NMR Spektren aus $\mathrm{D}_{2} \mathrm{O}$ zeigten jedoch keine Signale für die Methoxygruppe des Esters bzw. für den Carbonylkohlenstoff. Auch im IR Spektrum war keine Absorption im Bereich von Carbonylschwingungen zu erkennen. 
Die Wiederholung der ${ }^{13} \mathrm{C}$ NMR Spektren bei verschiedenen Feldstärken zeigte allerdings eine Aufspaltung der Signale, so daß nicht ausgeschlossen werden konnte, daß das Signal für den Methylkohlenstoff der Methoxygruppe mit dem Signal für die stickstoffgebundene Methylgruppe zusammenfällt und die Ringkohlenstoffatome im Imidazoliumion ebenfalls alle die gleiche Verschiebung aufweisen. Im ${ }^{1} \mathrm{H}$ NMR Spektrum wären dann die Signale der $\mathrm{N}$-gebundenen Methylgruppe zusammen mit denen der O-gebundenen Methylgruppe bei der gleichen chemischen Verschiebung zu sehen. Außerdem müßten N-Carbonyl-Wechselwirkungen die Schwingungsbande der Carbonylgruppe unterdrücken.

Um zu einem endgültigen Ergebnis zu gelangen, wurde die Verbindung aus Methanol kristallisiert und kristallographisch untersucht. Erste Ergebnisse der Strukturverfeinerung deuteten an, daß ein Dimer des Bis(N,N-dimethylimidazolium)quecksilber-(II)-chlorids $\mathbf{3}$ vorliegen könnte:

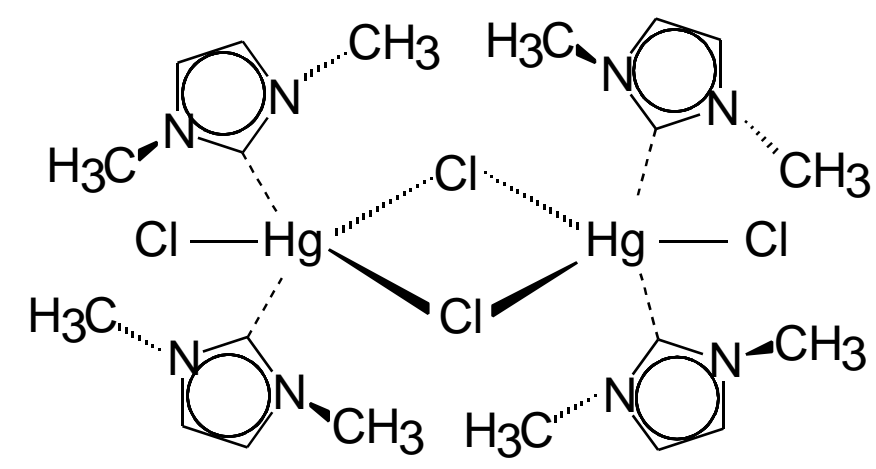

Abb. 32 
Dieses Ergebnis (Abb. 32) ließe sich problemlos mit den angefertigten Kernresonanz-, Infrarot- und Massenspektren erklären. Weil die Bildung von Methylchlorid aus dem Chlor-Ameisensäuremethylester nicht auszuschließen ist, mußte diese Möglichkeit also trotz des widersprüchlichen Ergebnisses der Elementaranalyse in Betracht gezogen werden.

Da das Monomer von 3 (Abb. 32) aber bereits beschrieben ${ }^{[31]}$ und die Bildung von Dimeren der Carbenkomplexe ebenfalls bekannt ist, ${ }^{[28 b]}$ erschien eine weitere Untersuchung dieser Verbindung nicht sinnvoll. Zusätzliche Probleme bei der Strukturverfeinerung führten deshalb zum Abbruch der Untersuchungen.

Es ist anzumerken, daß Verbindung 3, wie gewünscht, ein wasserlösliches Metallcarben darstellt. Das zu Beginn beschriebene Ziel ein wasserlösliches, katalytisch aktives Metallcarben darzustellen, konnte somit zum Teil erreicht werden. Darüber hinaus ist $\mathrm{zu}$ vermuten, daß eine aus N,N-Dimethylimidazoliumhydrochlorid und Palladiumacetat $\mathrm{zu}$ synthetisierende, dimere Carbenverbindung in Analogie zu 3 ebenfalls wasserlöslich sein sollte. Diese Verbindung könnte dann beide gewünschte Eigenschaften beinhalten und somit das Ergebnis vervollständigen.

Die Synthese einer wasserlöslichen, katalytisch aktiven Palladiumverbindung konnte im Rahmen dieser Arbeit jedoch nicht mehr durchgeführt werden. 


\section{Experimenteller Teil}

\subsection{Allgemeine Arbeitstechniken und Meßmethoden}

Die Handhabung von luft- und feuchtigkeitsempfindlichen Substanzen wurde unter Verwendung von Standard-Schlenk-Techniken in ausgeheizten, im Hochvakuum abgekühlten Glasgeräten unter trockener Stickstoff-Atmosphäre oder in der Inertgasumgebung einer Trockenbox durchgeführt.

Lösungsmittel wurden in der Regel über Kaliumhydroxid vorgetrocknet, vor dem Gebrauch von Kalium abdestilliert und im Vakuum entgast (Dichlormethan, Acetonitril von $\mathrm{P}_{4} \mathrm{O}_{10}$ ).

Deuterierte Lösungsmittel für die NMR-Spektroskopie wurden von Calciumdihydrid bzw. Natriumhydrid abdestilliert und unter StickstoffAtmosphäre in der Trockenbox gelagert. Ansonsten wurde nach gängigen Labormethoden getrocknet (z.B. Triethylamin). ${ }^{[32]}$

NMR-Spektren wurden auf den Geräten Bruker AM 200, AM 250, AS 400 oder MSL 400 bestimmt. Als externe Standards dienten TMS $\left({ }^{1} \mathrm{H},{ }^{13} \mathrm{C}\right.$ NMR) sowie 85 \%ige $\mathrm{H}_{3} \mathrm{PO}_{4}\left({ }^{31} \mathrm{P}\right.$ NMR). Heterokern-NMR-Spektren wurden ${ }^{1} \mathrm{H}$-breitbandentkoppelt. Die Messungen erfolgten bei RT. Lösungsmittel und Betriebsfrequenzen sind bei den jeweiligen Versuchsbeschreibungen angeführt. Die Multiplizitäten werden wie folgt abgekürzt: $s=$ Singulett, bs = breites Signal, $\mathrm{d}=$ Dublett, $\mathrm{t}=$ Triplett, $\mathrm{q}=$ Quartett, $\mathrm{m}=$ Multiplett, $(\mathrm{kl} .=$ schwaches Signal) . 
Die chemischen Verschiebungen werden als $\delta$-Werte in ppm angegeben; positive Vorzeichen bedeuten Tieffeldverschiebung, negative Vorzeichen Hochfeldverschiebung bezogen auf TMS als Standard $\left({ }^{31}\right.$ P-NMR-Spektren: $\left.\mathrm{H}_{3} \mathrm{PO}_{4}\right)$.

Infrarotspektren wurden mit dem Spektrometer BIO-RAD Digilab FTS-7 aufgenommen.

Alle Feststoffe wurden in getrocknetem Nujol verrieben und zwischen Kaliumbromid oder Natriumchloridplatten vermessen. Die Wellenzahlen $\tilde{v}_{\max }$ sind in $\mathrm{cm}^{-1}$ angegeben. Es werden ggf. nur starke und charakteristische Banden oberhalb von $\tilde{v}_{\max } 1500 \mathrm{~cm}^{-1}$ aufgeführt. Die Intensitäten werden dabei wie folgt abgekürzt: $s s t=$ sehr stark, $s t=$ stark, $s=$ schwach, $b=$ breit.

Massenspektren wurden auf den Geräten Finnigan MAT System 8230, Varian MAT CH 5 sowie Finnigan MAT 95 aufgenommen. Als Ionisierungsmethoden dienten sowohl Elektronenstoßionisation (EI) als auch Fast-Atom-Bombardement (FAB). Bei einer Isotopen-Signalverteilung wird nur der stärkste zugehörige Peak mit seiner relativen prozentualen Intensität angegeben.

Schmelz- und Zersetzungspunkte wurden in verschlossenen Glaskapillaren mit den Geräten Bühler SPA-1 oder HWS-SG 3000 bestimmt und sind nicht korrigiert. 
Elementaranalysen wurden im Hause vom analytischen Labor des Instituts für Anorganische Chemie der Universität Göttingen durchgeführt.

Röntgenstrukturanalysen wurden auf Stoe-Siemens-Vierkreisdiffraktometern bei den jeweils angegebenen Temperaturen an einem schockgekühlten Kristall im Öltropfen mit monochromatischer Mo- $\mathrm{K}_{\alpha}$-Strahlung (Wellenlänge 71.073 pm) angefertigt. Die Lösung und die Verfeinerung der Datensätze erfolgten mit Hilfe der Programme der SHELX-Gruppe in den entsprechenden Arbeitsgruppen im Haus. ${ }^{[33]}$

Alle Nicht-Wasserstoffatom-Lagen wurden (sofern sie nicht direkt in der Strukturlösung lokalisierbar waren) Differenzelektronendichtekarten im Laufe der Strukturermittlung entnommen und mit anisotropen Auslenkungsparametern verfeinert. Die Verfeinerung erfolgte nach dem „Vollmatrix Least-Squares Verfahren an $\mathrm{F}^{2}$ ". Wasserstoffatom-Lagen wurden geometrisch idealisiert, positioniert und nach dem Reitermodell verfeinert.

Gaschromatographische Untersuchungen wurden auf zwei verschiedenen Geräten durchgeführt. Im ersten Teil der Untersuchungen verwendete man einen Gaschromatographen Varian 3700 GC mit einer 30 Meter langen Kapillarsäule $\mathrm{J} \& W$ Scientific DB5. Der Säulendurchmesser betrug $0.25 \mathrm{~mm}$ und ist für Drücke bis 1.38 bar und Temperaturen im Bereich von $-60{ }^{\circ} \mathrm{C}$ bis $+325^{\circ} \mathrm{C}$ ausgelegt. Die Beschichtung hatte eine Dicke von 1 Micron. Die Anfangs-temperatur wurde auf $60{ }^{\circ} \mathrm{C}$ festgelegt und mit $10{ }^{\circ} \mathrm{C}$ pro Minute auf $260{ }^{\circ} \mathrm{C}$ erhöht. Die Injektortemperatur betrug $250{ }^{\circ} \mathrm{C}$, die des FID-Detektors $300^{\circ} \mathrm{C}$. 
Die Auswertung erfolgte mit Hilfe des Programms „Varian STAR 4.01, 1995, Chromatography Software“.

Im zweiten Teil der Untersuchungen verwendete man einen Gaschromatographen Hewlett Packard HP 6890 mit einer 30 Meter langen Kapillarsäule HP-5. Der Säuleninnendurchmesser betrug $0.32 \mathrm{~mm}$. Die Beschichtung hatte eine Dicke von $0.25 \mu \mathrm{m}$. Die Aufheizkurve wurde empirisch für die beste Trennwirkung optimiert. Man verwendete Helium als Trägergas und Wasserstoff als Brenngas. Die Injektortemperatur betrug $250{ }^{\circ} \mathrm{C}$, die des FIDDetektors $300{ }^{\circ} \mathrm{C}$. Die Integration und Auswertung erfolgte mit Hilfe der Gaschromatographen Software „HP ChemStations A.04.02, 1996“.

\section{Darstellung der Ausgangsverbindungen}

$\mathrm{Cp}^{*} \mathrm{TiCl}_{3}$ und $\mathrm{Cp} * \mathrm{TiMe}_{3}$ wurden nach Literaturmethoden dargestellt. ${ }^{[34]}$ Die tert-Butylphosphonsäure (Aldrich) wurde vor der Verwendung 24 Stunden lang im Vakuum getrocknet. Methylimidazol, Chlorameisensäureester und Quecksilberacetat wurden ohne weitere Bearbeitung eingesetzt. 


\subsection{Darstellung von $\left[\left(\mathrm{Cp}^{*} \mathrm{Ti}\right)_{3}\left(t-\mathrm{BuPO}_{3}\right)_{2}\left\{t-\mathrm{BuPO}_{2}(\mathrm{OH})\right\}(\mu-\mathrm{O})_{2}\right](1)$.}

$\mathrm{Zu}$ einer Lösung von $\mathrm{Cp}^{*} \mathrm{TiMe}_{3}(1 \mathrm{mmol}, 228 \mathrm{mg})$ in THF $(25 \mathrm{ml})$ wurde bei Raumtemperatur tropfenweise eine Lösung von tert-Butylphosphonsäure $(1 \mathrm{mmol}, 138 \mathrm{mg})$ in THF $(10 \mathrm{ml})$ zugegeben. Man rührte 12 Stunden lang und entfernte das Lösungsmittel im Vakuum. Der zurückgebliebene orange Feststoff wurde aus einem $\mathrm{CH}_{2} \mathrm{Cl}_{2} / n$-Hexan $(1: 1)$ Gemisch bei $-10{ }^{\circ} \mathrm{C}$ für 30 Tage rekristallisiert. Man erhielt Verbindung 1 als analytisch saubere Einkristalle. (Die NMR spektroskopische Untersuchung der Mutterlauge zeigte eine große Anzahl nicht identifizierbarer Nebenprodukte.)

Ausbeute: $65 \mathrm{mg}(20 \%, 0.06 \mathrm{mmol})$.

Schmelzpunkt $>300{ }^{\circ} \mathrm{C}$ (Zersetzung).

Elementaranalyse für $\mathrm{C}_{42} \mathrm{H}_{73} \mathrm{O}_{11} \mathrm{P}_{3} \mathrm{Ti}_{3}$ (990.6):

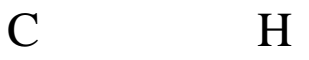

Ber. $(\%): \quad 50.9 \quad 7.4$

Gef. (\%): $\quad 50.3 \quad 7.7$

Massenspektrum (EI, $70 \mathrm{eV}): \mathrm{m} / \mathrm{z} 990\left(2 \%, \mathrm{M}^{+}\right), 135\left(100 \%, \mathrm{Cp}^{*}\right)$.

IR-Spektrum (KBr): $\tilde{v}\left[\mathrm{~cm}^{-1}\right]$ 3420, 1261, 1153, 1079, 1056, 1021, 973, 946, 800, $723,622$. 
${ }^{1} \mathrm{H}$ NMR Spektrum (250 MHz, $\left.\mathrm{CDCl}_{3}\right): \delta[\mathrm{ppm}]$ 1.15-1.25 (m, 27H, $\left.\mathrm{C}\left(\mathrm{CH}_{3}\right)_{3}\right)$, $1.99,2.10\left(2: 1, \mathrm{~s}, 45 \mathrm{H},\left(\mathrm{C}_{5}\left(\mathrm{CH}_{3}\right)_{5}\right)\right.$.

${ }^{31} \mathrm{P}$ NMR Spektrum $\left(101 \mathrm{MHz}, \mathrm{CDCl}_{3}\right)$ : $\delta[\mathrm{ppm}] 31.9\left(\mathrm{P}_{2}\right), 35.6$ und $35.9\left(\mathrm{P}_{1}\right),(\mathrm{s}$, $1: 1: 1)$.

\subsection{Darstellung von $\left[\left(\mathrm{Cp}^{*} \mathrm{TiCl}\right)_{2}(t-\mathrm{BuPOH})\left\{t-\mathrm{BuPO}_{2}\right\}(\mu-\mathrm{O})_{3}\right](2)$.}

$\mathrm{Zu}$ einer Lösung von tert-Butylphosphonsäure (2.9 mmol, $396 \mathrm{mg})$ und Triethylamin $(5.7 \mathrm{mmol}, 0.8 \mathrm{ml})$ in THF $(20 \mathrm{ml})$ tropfte man eine Lösung von $\mathrm{Cp}^{*} \mathrm{TiCl}_{3}(2.4 \mathrm{mmol}, 687 \mathrm{mg}$, Überschuß) in THF $(20 \mathrm{ml})$. Man rührte 12 Stunden lang und entfernte das Lösungsmittel im Vakuum. Der zurückgebliebene rotbraune, zähe Rückstand wurde in $\mathrm{CDCl}_{3} \mathrm{NMR}$ spektroskopisch untersucht und aus $\mathrm{CHCl}_{3}$ bei $\mathrm{RT}$ rekristallisiert. Man erhielt Verbindung 2 als analytisch saubere Einkristalle. Die erneute NMR spektroskopische Untersuchung der im Vakuum getrockneten Kristalle in deuteriertem Trichlormethan führte zu keinem verwertbaren Ergebnis, da sich 2 nicht mehr vollständig auflöste. Auch in deuteriertem Benzol erhielt man nur eine dunkelorange Lösung mit gelben und leuchtend roten ungelösten Anteilen. Eine Ausbeute wurde nicht bestimmt, da nicht auszuschließen ist, daß nach der Rekristallisation ein Produktgemisch vorliegt, aus dem nur $\mathbf{2}$ kristallisiert ist.

Zersetzungsbereich $>130{ }^{\circ} \mathrm{C}$. (Es kondensiert eine orange Flüssigkeit aus der Probe, ohne daß die Probe eine beobachtbare Volumenänderung erfährt. Die Probe färbt sich dabei langsam schwarz.) 
Elementaranalyse für $\mathrm{C}_{28} \mathrm{H}_{51} \mathrm{Cl}_{2} \mathrm{O}_{7} \mathrm{P}_{2} \mathrm{Ti}_{2}$ (728.32):

(getrocknetes Reaktionsgemisch)

$\begin{array}{llll}\mathrm{C} & \mathrm{H} & \mathrm{Cl} & \mathrm{P}\end{array}$

$\begin{array}{lllll}\text { Ber. }(\%): & 46.1 & 7.1 & 9.7 & 8.5\end{array}$

Gef. $(\%): \quad 45.9 \quad 6.9 \quad 9.7 \quad 8.6$

Massenspektrum (EI, $70 \mathrm{eV})$ : (getrocknetes Reaktionsgemisch)

$\mathrm{m} / \mathrm{z} 855\left(60 \%, \mathrm{M}(\mathbf{1})^{+}-\mathrm{Cp}^{*}\right), 121\left(100 \%, \mathrm{Cp}^{*}-\mathrm{CH}_{2}\right)$.

${ }^{1} \mathrm{H}$ NMR Spektrum (200 MHz, $\mathrm{CDCl}_{3}$ ): (getrocknetes Reaktionsgemisch)

$\delta[\mathrm{ppm}] 1.05-1.30\left(\mathrm{~m}, 27 \mathrm{H}, \mathrm{C}\left(\mathrm{CH}_{3}\right)_{3}\right), 1.83(\mathrm{~m}, \mathrm{THF}), 2.04-2.18(\mathrm{~m}, 30 \mathrm{H}$, $\left(\mathrm{C}_{5}\left(\mathrm{CH}_{3}\right)_{5}\right), 3.72(\mathrm{~m}, \mathrm{THF})$.

${ }^{31}$ P NMR Spektrum (161.96 MHz, $\mathrm{CDCl}_{3}$ ): (getrocknetes Reaktionsgemisch)

$\delta$ [ppm] 23.5 (kl.), 32.8 (kl.), 34.9 (kl.), 37.1, 37.3, 37.7 (kl.), 37.9, 39.6, 39.9 (kl.), 41.8 (kl.), 42.0, 42.4(kl.), 45.1 (kl.), 46.3(kl.).

IR-Spektrum $(\mathrm{NaCl})$ : (getrocknete Kristalle)

$\tilde{v}\left[\mathrm{~cm}^{-1}\right] 3162,3138,1261,1106,1089,1060,1036,1016,987,973,924,803$, $751,735,659,644,600$.

${ }^{1} \mathrm{H}$ NMR Spektrum $\left(200 \mathrm{MHz}, \mathrm{CDCl}_{3}\right)$ : (getrocknete Kristalle)

$\delta[\mathrm{ppm}] 1.05-1.30\left(\mathrm{~m}, 27 \mathrm{H}, \mathrm{C}\left(\mathrm{CH}_{3}\right)_{3}\right), 2.04-2.18\left(\mathrm{~m}, 30 \mathrm{H},\left(\mathrm{C}_{5}\left(\mathrm{CH}_{3}\right)_{5}\right)\right.$. 
${ }^{31} \mathrm{P}$ NMR Spektrum $\left(161.96 \mathrm{MHz}, \mathrm{CDCl}_{3}\right)$ : (getrocknete Kristalle)

$\delta$ [ppm] 37.1, 37.3 , 37.9, 39.6, 42.0 (und zahlreiche kleinere Signale).

${ }^{13} \mathrm{C}$ NMR Spektrum (100 MHz, $\mathrm{C}_{6} \mathrm{D}_{6}$ ): (getrocknete Kristalle)

$\delta[\mathrm{ppm}] 1.3,11.5$ - 13.1, 25.5-26.4, 29.3 - 32.3, 77.6, 123.1, 126.1 - 128.9.

\subsection{Darstellung von $\left(\mathrm{C}_{10} \mathrm{H}_{16} \mathrm{Cl}_{2} \mathrm{Hg}_{1} \mathrm{~N}_{4}\right)_{2}(3)$}

$\mathrm{Zu}$ einer Lösung von $\mathrm{N}$-Methylimidazol (32.35 mmol, $2.66 \mathrm{~g}, 2.58 \mathrm{ml}$ ) in Dichlormethan $(10 \mathrm{ml})$ tropfte man bei RT eine Lösung von Ameisensäuremethylester (32.35 mmol, 3.06 g, $2.50 \mathrm{ml}$ ) in Dichlormethan (10 $\mathrm{ml}$ ). Man rührte eine weitere Stunde, filtrierte den gebildeten Feststoff (Hydrochlorid) ab und trocknete den Feststoff im Vakuum. Dem gelben Feststoff wurde eine Probe zur Analyse entnommen.

Man löste $1.77 \mathrm{~g}$ des Feststoffes in DMSO (6 ml) und tropfte bei RT eine Lösung von Quecksilberacetat (5 mmol, $1.59 \mathrm{~g})$ in DMSO $(5 \mathrm{ml})$ zu. Das Reaktionsgemisch wurde zur Vervollständigung der Reaktion unter Rühren für 10 Min. auf $80{ }^{\circ} \mathrm{C}$ erhitzt. Es fiel ein weißer Feststoff aus, der abfiltriert und mit $n$-Pentan (10 ml) und anschließend zweimal mit ungetrocknetem Diethylether (10 ml) gewaschen wurde. Die Rekristallisation aus Methanol ergab farblose Einkristalle von 3. 
Zwischenprodukt: (gelber Feststoff)

Massenspektrum (EI, $70 \mathrm{eV})$ :

$\mathrm{m} / \mathrm{z} 82$ (100\%, Methylimidazol).

IR-Spektrum (KBr): $\tilde{v}\left[\mathrm{~cm}^{-1}\right] 3383$ b, 3141 st, 1799 s, 1730 s, 1696 s, 1669 s, 1575 st, 1340 s, 1175 st, 1125 s, 1029 st, 866 st, 803 st, 626 sst.

${ }^{1} \mathrm{H}$ NMR Spektrum (200 MHz, $\mathrm{D}_{2} \mathrm{O}$ ): $\delta$ [ppm] 3.31-3.32 (d, $\left.6 \mathrm{H}, \mathrm{N}-\mathrm{CH}_{3}\right), 4.26$ (s, $\left.\mathrm{H}_{2} \mathrm{O}\right), 6.84-6.85\left(\mathrm{~d}, 2 \mathrm{H}, \mathrm{C}_{2} \mathrm{H}_{2}\right), 7.92$ und $8.08\left(\mathrm{~s}, 1 \mathrm{H}, \mathrm{C}^{+}-\mathrm{H}\right)$.

${ }^{13} \mathrm{C}$ NMR Spektrum (100 MHz, $\left.\mathrm{D}_{2} \mathrm{O}\right): \delta[\mathrm{ppm}] 36.42$ und 36.45( $\left(\mathrm{CH}_{3}\right), 124.21 \&$ $124.25(\mathrm{C}=\mathrm{C}), 137.39\left(\mathrm{C}^{+}\right)$.

Quecksilberacetat (zum Vergleich):

${ }^{1} \mathrm{H}$ NMR Spektrum(200 MHz, $\left.\mathrm{D}_{2} \mathrm{O}\right): \delta[\mathrm{ppm}] 1.53\left(\mathrm{CH}_{3}\right)$.

${ }^{13}$ C NMR Spektrum(100 MHz, $\left.\mathrm{D}_{2} \mathrm{O}\right): \delta[\mathrm{ppm}] 20.9(\mathrm{C}=\mathrm{O}), 178.9\left(\mathrm{CH}_{3}\right)$. 
Verbindung $\mathrm{C}_{20} \underline{\mathrm{H}}_{32} \underline{\mathrm{Cl}}_{4} \mathrm{Hg}_{2} \underline{\mathrm{N}}_{8}$ (3) (927.52):

Elementaranalyse für " $\mathrm{C}_{12} \mathrm{H}_{16} \mathrm{Cl}_{2} \mathrm{HgN}_{4} \mathrm{O}_{4} \cdot 2 \mathrm{H}_{2} \mathrm{O}$ (587.78)“:

$\begin{array}{llllll} & \mathrm{C} & \mathrm{H} & \mathrm{Cl} & \mathrm{N} & \mathrm{Hg} \\ \text { Ber. (\%): } & 24.5 & 3.4 & 12.1 & 9.5 & 34.1 \\ \text { Gef. (\%): } & 23.8 & 4.0 & 12.8 & 9.9 & 36.7\end{array}$

Massenspektrum (EI, $70 \mathrm{eV}): 97.1\left(100 \%\right.$, ?), $333.1\left(20 \%, \mathrm{C}_{5} \mathrm{H}_{8} \mathrm{Cl}_{1} \mathrm{~N}_{2} \mathrm{Hg}_{1}\right)$, $429.3\left(20 \%, 1 / 2 \mathrm{M}^{+}-\mathrm{Cl}\right), 797.5\left(3 \%, \mathrm{C}_{15} \mathrm{H}_{24} \mathrm{Cl}_{3} \mathrm{~N}_{6} \mathrm{Hg}_{2}\right)$.

IR-Spektrum (KBr): $\tilde{v}\left[\mathrm{~cm}^{-1}\right] 3383$ b, 3146 s, 3093 s, 1733 s, 1632 st, 1619 st, 1570 st, 1345 s, 1235 st, 1095 s, 1019 st, 953 s, 775 st, 742 st, 722 st, 663 s.

${ }^{1} \mathrm{H}$ NMR Spektrum (200 MHz, $\left.\mathrm{D}_{2} \mathrm{O}\right): \delta$ [ppm] $3.46\left(\mathrm{~s}, 12 \mathrm{H}, \mathrm{CH}_{3}\right), 4.27\left(\mathrm{H}_{2} \mathrm{O}\right)$, $6.91\left(\mathrm{~s}, 4 \mathrm{H}, \mathrm{C}_{2} \mathrm{H}_{2}\right)$.

${ }^{13} \mathrm{C}$ NMR Spektrum $\left(100 \mathrm{MHz}, \mathrm{D}_{2} \mathrm{O}\right): \delta[\mathrm{ppm}] 38.5\left(\mathrm{CH}_{3}\right), 125.8(\mathrm{C}=\mathrm{C}), 176.1$ (C-Hg). 


\section{Behandlung und Entsorgung der Abfälle}

Alle Abfälle wurden, soweit sie nicht zur Wiederverwendung aufgearbeitet werden konnten, deklariert und in entsprechend gekennzeichneten Abfallbehältern zentral entsorgt.

Lösungsmittel wurden dabei im allgemeinen im Vakuum von den Reaktionsgemischen abgetrennt und mit flüssigem Stickstoff in Kühlfallen kondensiert. Insoweit eine destillative Aufarbeitung nicht möglich war, wurden sie getrennt nach halogenhaltigen und halogenfreien Lösungsmitteln entsorgt. Metallsalzlösungen wurden weitestgehend eingeengt und getrennt nach schwermetallhaltigen und schwermetallfreien Lösungen entsorgt. Reaktive Chemikalienrückstände wurden durch vorsichtige Hydrolyse inaktiviert, Säuren und Basen neutralisiert und entsprechend ihrer Zusammensetzung entsorgt. Das zur Entfettung verwendete alkalische Reinigungsbad wurde eingeengt und wegen des Schwermetall- und Halogengehalts gesondert deklariert und entsorgt.

Das säurehaltige Entkalkungsbad wurde mit Natriumcarbonatlösung neutralisiert und konnte aufgrund der geringen Belastung in das kommunale Abwassernetz entsorgt werden.

\section{Angefallene Abfallmengen:}

Halogenhaltige Lösungsmittelabfälle 4 Liter

Halogenfreie Lösungsmittelabfälle $\quad 30$ Liter

Säureabfälle

20 Liter

Basenabfälle

15 Liter

Schwermetallhaltige Abfälle

5 Liter

Schwermetallfreie Abfälle

2 Liter 


\section{GC-Daten-Tabellen zu den Abbildungen}

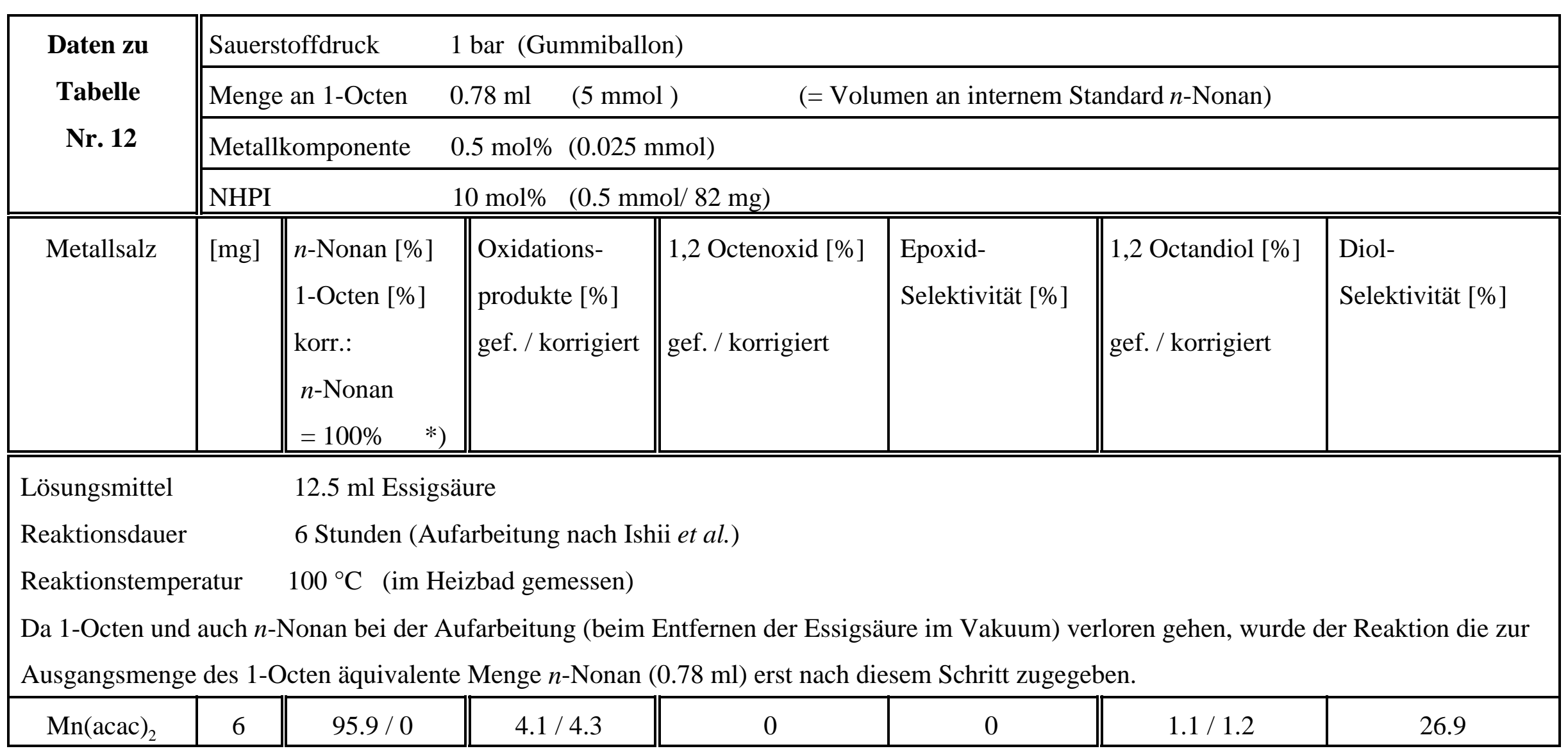




\begin{tabular}{|c|c|c|c|c|c|c|c|}
\hline $\begin{array}{c}\mathrm{Cu}(\mathrm{OAc})_{2} \\
\cdot \mathrm{H}_{2} \mathrm{O}\end{array}$ & 6 & $85.6 / 0$ & $14.4 / 16.8$ & $1.8 / 2.1$ & 12.5 & $3.1 / 3.6$ & 21.5 \\
\hline $\mathrm{VO}\left(\mathrm{OSiPh}_{3}\right)_{3}$ & 21 & $88.5 / 0$ & $11.5 / 13.0$ & $1.6 / 1.8$ & 13.9 & $5.7 / 6.4$ & 49.5 \\
\hline \multicolumn{2}{|l|}{ Lösungsmittel } & \multicolumn{6}{|c|}{ 12.5 ml Benzonitril (Peak bei 7.77 Min. Retentionszeit nicht mit integriert) } \\
\hline \multicolumn{2}{|c|}{ Reaktionsdauer } & \multicolumn{6}{|c|}{6 Stunden (ohne Aufarbeitung, mit Pentan verdünnt, gaschromatographisch untersucht) } \\
\hline \multicolumn{2}{|c|}{ Reaktionstemperatur } & \multicolumn{6}{|c|}{$100^{\circ} \mathrm{C} \quad$ (im Heizbad gemessen) } \\
\hline $\operatorname{Mn}(\mathrm{acac})_{2}$ & 6 & $44.5 / 42.4$ & $13.0 / 29.3$ & $0.2 / 0.4$ & 0.0 & 0 & 0 \\
\hline \multicolumn{8}{|c|}{ Lösungsmittel $\quad 12.5 \mathrm{ml}$ tert-Butanol } \\
\hline \multicolumn{2}{|c|}{ Reaktionsdauer } & \multicolumn{6}{|c|}{6 Stunden (ohne Aufarbeitung, mit Pentan verdünnt, gaschromatographisch untersucht) } \\
\hline \multicolumn{2}{|c|}{ Reaktionstemperatur } & \multicolumn{6}{|c|}{$60{ }^{\circ} \mathrm{C} \quad$ (im Heizbad gemessen) } \\
\hline $\operatorname{Mn}(\operatorname{acac})_{2}$ & 6 & $49.3 / 49.5$ & $0.2 / 0.4$ & 0 & 0 & 0 & 0 \\
\hline $\begin{array}{c}\mathrm{Cu}(\mathrm{OAc})_{2} \\
\cdot \mathrm{H}_{2} \mathrm{O}\end{array}$ & 5 & $50.1 / 49.9$ & 0 & 0 & 0 & 0 & 0 \\
\hline \multicolumn{8}{|c|}{6 Stunden (ohne Aufarbeitung, mit Pentan verdünnt, gaschromatographisch untersucht) } \\
\hline \multicolumn{2}{|c|}{ Reaktionstemperatur } & \multicolumn{6}{|l|}{ Rückfluß } \\
\hline $\operatorname{Mn}(\operatorname{acac})_{2}$ & 6 & $54.8 / 38.2$ & $7.0 / 12.8$ & $0.2 / 0.3$ & 2.6 & $0.2 / 0.3$ & 2.6 \\
\hline
\end{tabular}




\begin{tabular}{|c|c|c|c|c|c|c|c|}
\hline Metallsalz & {$[\mathrm{mg}]$} & $\begin{array}{l}n \text {-Nonan [\%] } \\
1 \text {-Octen [\%] } \\
\text { korr.: } \\
n \text {-Nonan } \\
=100 \% \quad *)\end{array}$ & $\begin{array}{l}\text { Oxidations- } \\
\text { produkte [\%] } \\
\text { gef. / korrigiert }\end{array}$ & $\begin{array}{l}1,2 \text { Octenoxid [\%] } \\
\text { gef. / korrigiert }\end{array}$ & $\begin{array}{l}\text { Epoxid- } \\
\text { Selektivität [\%] }\end{array}$ & $\begin{array}{l}\text { 1,2 Octandiol [\%] } \\
\text { gef. / korrigiert }\end{array}$ & $\begin{array}{l}\text { Diol- } \\
\text { Selektivität [\%] }\end{array}$ \\
\hline $\begin{array}{c}\mathrm{Cu}(\mathrm{OAc})_{2} \\
\cdot \mathrm{H}_{2} \mathrm{O} \\
\end{array}$ & 5 & $50.9 / 45.5$ & $3.6 / 7.1$ & 0 & 0 & 0 & 0 \\
\hline \multicolumn{8}{|c|}{ Lösungsmittel $\quad 12.5 \mathrm{ml}$ Acetonitril } \\
\hline \multicolumn{2}{|c|}{ Reaktionsdauer } & \multicolumn{6}{|c|}{6 Stunden (ohne Aufarbeitung, mit Pentan verdünnt, gaschromatographisch untersucht) } \\
\hline \multicolumn{2}{|c|}{ Reaktionstemperatur } & \multicolumn{6}{|c|}{$80{ }^{\circ} \mathrm{C}$ (im Heizbad gemessen) } \\
\hline $\operatorname{Mn}(\mathrm{acac})_{2}$ & 6 & $49.9 / 46.6$ & $3.5 / 7.0$ & 0 & 0 & 0 & 0 \\
\hline $\begin{array}{c}\mathrm{Cu}(\mathrm{OAc})_{2} \\
\cdot \mathrm{H}_{2} \mathrm{O}\end{array}$ & 5 & $52.4 / 47.1$ & $0.5 / 1.1$ & 0 & 0 & 0 & 0 \\
\hline $\mathrm{VO}(\mathrm{acac})_{2}$ & 7 & $39.0 / 60.2$ & $0.9 / 2.3$ & 0 & 0 & 0 & 0 \\
\hline \multicolumn{8}{|c|}{ Reaktionsdauer $\quad 6$ Stunden (ohne Aufarbeitung, mit Pentan verdünnt, gaschromatographisch untersucht) } \\
\hline \multicolumn{2}{|c|}{ Reaktionstemperatur } & \multicolumn{6}{|l|}{ Rückfluß } \\
\hline $\operatorname{Mn}(\mathrm{acac})_{2}$ & 6 & $55.2 / 41.5$ & $3.3 / 5.9$ & $0.8 / 1.5$ & 25.4 & 0 & 0 \\
\hline $\begin{array}{c}\mathrm{Cu}(\mathrm{OAc})_{2} \\
\cdot \mathrm{H}_{2} \mathrm{O}\end{array}$ & 5 & $49.7 / 49.3$ & $1.0 / 2.0$ & 0 & 0 & 0 & 0 \\
\hline
\end{tabular}




\begin{tabular}{|c|c|c|c|c|c|c|c|}
\hline $\mathrm{VO}(\mathrm{acac})_{2}$ & 7 & $58.9 / 40.7$ & $0.4 / 0.7$ & 0 & 0 & 0 & 0 \\
\hline \multicolumn{8}{|c|}{ Lösungsmittel $\quad 12.5 \mathrm{ml}$ Dichlormethan } \\
\hline \multicolumn{2}{|c|}{ Reaktionsdauer } & \multicolumn{6}{|c|}{ 6.5 Stunden (ohne Aufarbeitung, mit Pentan verdünnt, gaschromatographisch untersucht) } \\
\hline \multicolumn{2}{|c|}{ Reaktionstemperatur } & Rückfluß & & & & & \\
\hline $\mathrm{Mn}(\mathrm{acac})_{2}$ & 6 & $50.6 / 49.4$ & 0 & 0 & 0 & 0 & 0 \\
\hline $\begin{array}{c}\mathrm{Cu}(\mathrm{OAc})_{2} \\
\cdot \mathrm{H}_{2} \mathrm{O}\end{array}$ & 5 & $49.8 / 49.8$ & $0.4 / 0.8$ & 0 & 0 & 0 & 0 \\
\hline $\mathrm{VO}(\mathrm{acac})_{2}$ & 7 & $50.5 / 49.2$ & $0.3 / 0.6$ & 0 & 0 & 0 & 0 \\
\hline
\end{tabular}

- Molprozent Angaben beziehen sich auf 1-Octen (=100\%)

- Oxidationsprodukte $=$ Produkte mit einer Retentionszeit größer als 1-Octen

- Korrektur $=$ Auswertung mit $n$-Nonan als internem Standard entsprechend $100 \%$ eingesetztem 1-Octen

*) Bei den Versuchen mit direkter Untersuchung des Reaktionsgemisches sollte der interne Standard $n$-Nonan zu ca. 50 Prozent neben unverbrauchtem 1-Octen $(<50 \%)$ und seinen Folgeprodukten vorliegen. Es zeigte sich jedoch, daß der Anteil an internem Standard abnahm. Dies wird auf die Oxidation des Standards zurückgeführt. 


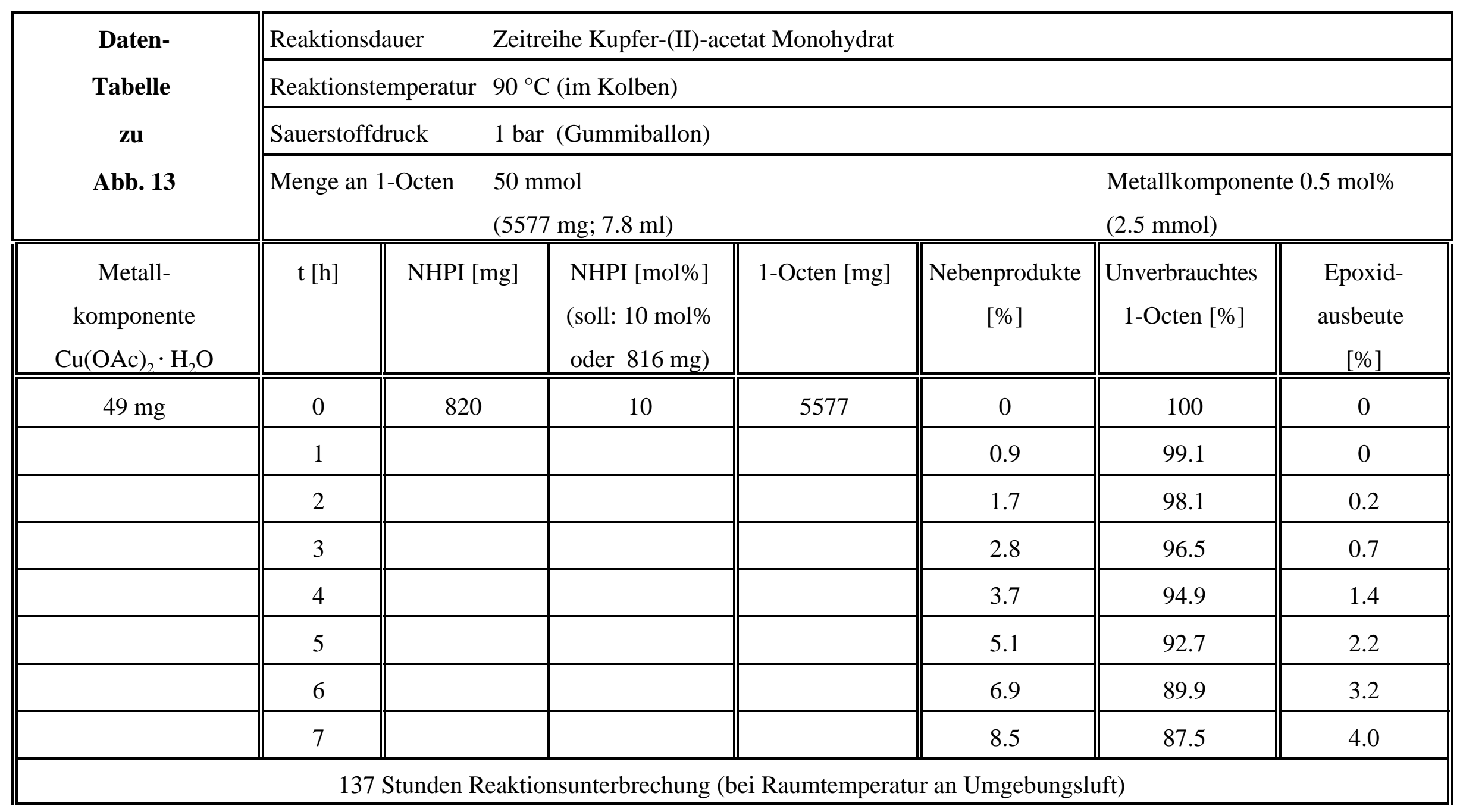




\begin{tabular}{|c|c|c|c|c|c|c|c|}
\hline $\begin{array}{c}\text { Metall- } \\
\text { komponente } \\
\mathrm{Cu}(\mathrm{OAc})_{2} \cdot \mathrm{H}_{2} \mathrm{O} \\
\end{array}$ & $\mathrm{t}[\mathrm{h}]$ & NHPI [mg] & $\begin{array}{l}\text { NHPI [mol\%] } \\
\text { (soll: } 10 \mathrm{~mol} \% \\
\text { oder } 816 \mathrm{mg} \text { ) } \\
\end{array}$ & 1-Octen [mg] & $\begin{array}{c}\text { Nebenprodukte } \\
{[\%]}\end{array}$ & \begin{tabular}{|c} 
Unverbrauchtes \\
$1-$ Octen [\%]
\end{tabular} & $\begin{array}{c}\text { Epoxid- } \\
\text { ausbeute } \\
{[\%]} \\
\end{array}$ \\
\hline & 8 & & & & 5.9 & 90.2 & 3.9 \\
\hline & 9 & & & & 8.6 & 85.9 & 5.2 \\
\hline & 10 & & & & 5.9 & 87.9 & 6.2 \\
\hline & 11 & & & & 11.3 & 81.3 & 7.4 \\
\hline & 12 & & & & 12.8 & 78.3 & 8.9 \\
\hline & 13 & & & & 14.0 & 76.5 & 9.5 \\
\hline & 14 & & & & 13.0 & 77.1 & 9.9 \\
\hline
\end{tabular}




\begin{tabular}{|c|c|c|c|c|c|c|c|}
\hline Daten- & \multicolumn{2}{|c|}{ Reaktionsdauer } & \multicolumn{5}{|c|}{ Zeitreihe Manganacetylacetonat } \\
\hline Tabelle & \multicolumn{7}{|c|}{ Reaktionstemperatur $90^{\circ} \mathrm{C}$ (im Kolben) } \\
\hline & \multicolumn{7}{|c|}{ Sauerstoffdruck 1 bar (Gummiballon) } \\
\hline Abb. 14 & \multicolumn{2}{|c|}{ Menge an 1-Octen } & \multicolumn{2}{|c|}{$\begin{array}{l}50 \mathrm{mmol} \\
(5577 \mathrm{mg} ; 7.8 \mathrm{ml})\end{array}$} & & \multicolumn{2}{|c|}{$\begin{array}{l}\text { Metallkomponente } 0.5 \mathrm{~mol} \% \\
(2.5 \mathrm{mmol})\end{array}$} \\
\hline $\begin{array}{c}\text { Metall- } \\
\text { komponente } \\
\mathrm{Mn}(\mathrm{acac})_{2}\end{array}$ & $\mathrm{t}[\mathrm{h}]$ & NHPI $[\mathrm{m}$ & $\begin{array}{l}\text { NHPI [mol\%] } \\
\text { (soll: } 10 \mathrm{~mol} \% \\
\text { oder } 816 \mathrm{mg} \text { ) }\end{array}$ & 1-Octen $[\mathrm{mg}]$ & $\begin{array}{c}\text { Nebenprodukte } \\
{[\%]}\end{array}$ & $\begin{array}{c}\text { Unverbrauchtes } \\
1 \text {-Octen [\%] }\end{array}$ & $\begin{array}{c}\text { Epoxid- } \\
\text { ausbeute } \\
{[\%]} \\
\end{array}$ \\
\hline \multirow[t]{8}{*}{$63 \mathrm{mg}$} & 0 & 820 & 10 & 5577 & 0 & 100 & 0 \\
\hline & 1 & & & & 4.4 & 93.4 & 2.2 \\
\hline & 2 & & & & 5.8 & 95.8 & 1.6 \\
\hline & 3 & & & & 5.2 & 92.6 & 2.2 \\
\hline & 4 & & & & 3.9 & 93.8 & 2.3 \\
\hline & 5 & & & & 5.1 & 92.3 & 2.6 \\
\hline & 6 & & & & 4.9 & 92.1 & 3.0 \\
\hline & 7 & & & & 10.9 & 86.3 & 2.8 \\
\hline
\end{tabular}




\begin{tabular}{|c|c|c|c|c|c|c|c|}
\hline $\begin{array}{c}\text { Metall- } \\
\text { komponente } \\
\mathrm{Mn}(\mathrm{acac})_{2} \\
\end{array}$ & $\mathrm{t}[\mathrm{h}]$ & NHPI [mg] & $\begin{array}{l}\text { NHPI [mol\%] } \\
\text { (soll: } 10 \mathrm{~mol} \% \\
\text { oder } 816 \mathrm{mg} \text { ) } \\
\end{array}$ & 1-Octen $[\mathrm{mg}]$ & $\begin{array}{c}\text { Nebenprodukte } \\
{[\%]}\end{array}$ & $\begin{array}{c}\text { Unverbrauchtes } \\
\text { 1-Octen [\%] }\end{array}$ & $\begin{array}{c}\text { Epoxid- } \\
\text { ausbeute } \\
{[\%]} \\
\end{array}$ \\
\hline & 8 & & & & 7.6 & 87.5 & 4.9 \\
\hline & 9 & & & & 11.9 & 80.7 & 7.4 \\
\hline & 10 & & & & 4.4 & 87.4 & 8.2 \\
\hline & 11 & & & & 6.7 & 82.6 & 10.7 \\
\hline & 12 & & & & 19.5 & 68.5 & 12.0 \\
\hline & 13 & & & & 23.1 & 66.1 & 10.8 \\
\hline & 14 & & & & 6.5 & 83.2 & 10.3 \\
\hline
\end{tabular}




\begin{tabular}{|c|c|c|c|c|c|c|c|}
\hline Daten- & \multicolumn{2}{|c|}{ Reaktionsdauer } & \multicolumn{5}{|c|}{ Zeitreihe Vanadylacetylacetonat } \\
\hline Tabelle & \multicolumn{7}{|c|}{ Reaktionstemperatur $90^{\circ} \mathrm{C}$ (im Kolben) } \\
\hline & \multicolumn{2}{|c|}{ Sauerstoffdruck } & \multicolumn{5}{|c|}{1 bar (Gummiballon) } \\
\hline Abb. 15 & \multicolumn{2}{|c|}{ Menge an 1-Octen } & \multicolumn{2}{|c|}{$\begin{array}{l}50 \mathrm{mmol} \\
(5577 \mathrm{mg} ; 7.8 \mathrm{ml}) \\
\end{array}$} & & \multicolumn{2}{|c|}{$\begin{array}{l}\text { Metallkomponente } 0.5 \mathrm{~mol} \% \\
(2.5 \mathrm{mmol})\end{array}$} \\
\hline $\begin{array}{c}\text { Metall- } \\
\text { komponente } \\
\mathrm{VO}(\mathrm{acac})_{2} \\
\end{array}$ & $\mathrm{t}[\mathrm{h}]$ & NHPI $[\mathrm{m}$ & $\begin{array}{l}\text { NHPI [mol\%] } \\
\text { (soll: } 10 \mathrm{~mol} \% \\
\text { oder } 816 \mathrm{mg} \text { ) } \\
\end{array}$ & 1-Octen $[\mathrm{mg}]$ & $\begin{array}{c}\text { Nebenprodukte } \\
{[\%]}\end{array}$ & $\begin{array}{c}\text { Unverbrauchtes } \\
1-\text { Octen [\%] }\end{array}$ & $\begin{array}{c}\text { Epoxid- } \\
\text { ausbeute } \\
{[\%]} \\
\end{array}$ \\
\hline \multirow[t]{8}{*}{$66 \mathrm{mg}$} & 0 & 820 & 10 & 5577 & 0 & 100 & 0 \\
\hline & 1 & & & & 0.3 & 99.7 & 0 \\
\hline & 2 & & & & 1.7 & 98.0 & 0.3 \\
\hline & 3 & & & & 2.0 & 97.9 & 0.1 \\
\hline & 4 & & & & 1.9 & 98.1 & 0.0 \\
\hline & 5 & & & & 2.4 & 97.2 & 0.4 \\
\hline & 6 & & & & 2.2 & 97.0 & 0.8 \\
\hline & 7 & & & & 5.3 & 92.4 & 2.3 \\
\hline
\end{tabular}




\begin{tabular}{|c|c|c|c|c|c|c|c|}
\hline $\begin{array}{c}\text { Metall- } \\
\text { komponente } \\
\mathrm{VO}(\mathrm{acac})_{2} \\
\end{array}$ & $\mathrm{t}[\mathrm{h}]$ & NHPI [mg] & $\begin{array}{l}\text { NHPI [mol\%] } \\
\text { (soll: } 10 \mathrm{~mol} \% \\
\text { oder } 816 \mathrm{mg} \text { ) } \\
\end{array}$ & 1-Octen [mg] & $\begin{array}{c}\text { Nebenprodukte } \\
{[\%]}\end{array}$ & $\begin{array}{c}\text { Unverbrauchtes } \\
1 \text {-Octen [\%] }\end{array}$ & $\begin{array}{c}\text { Epoxid- } \\
\text { ausbeute } \\
{[\%]} \\
\end{array}$ \\
\hline & 8 & & & & 3.1 & 95.1 & 1.8 \\
\hline & 9 & & & & 8.4 & 89.8 & 1.8 \\
\hline & 10 & & & & 10.3 & 88.6 & 1.1 \\
\hline & 11 & & & & 12.7 & 86.2 & 1.1 \\
\hline & 12 & & & & 14.3 & 84.6 & 1.1 \\
\hline & 13 & & & & 20.3 & 78.4 & 1.3 \\
\hline & 14 & & & & 16.0 & 82.5 & 1.5 \\
\hline
\end{tabular}




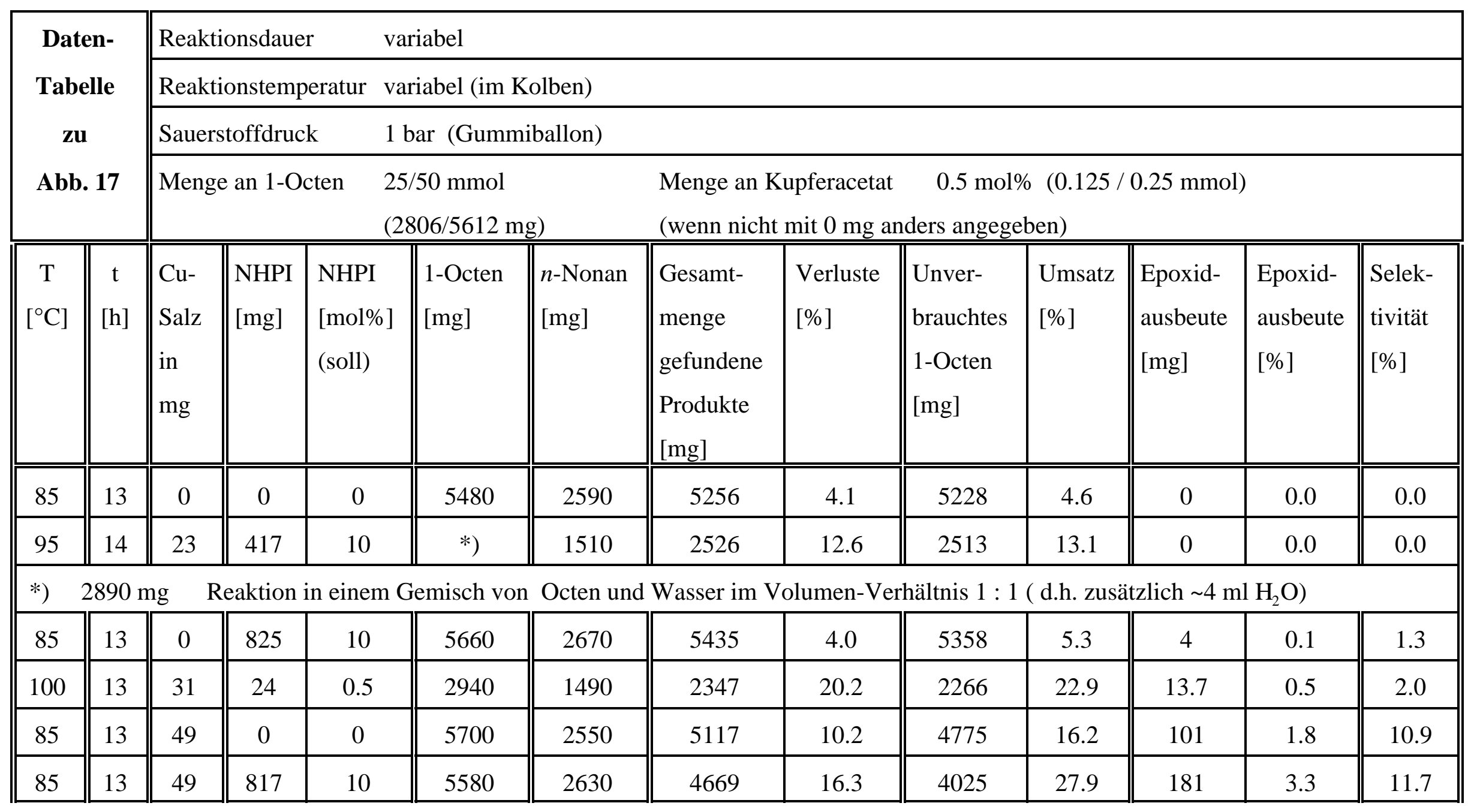




\begin{tabular}{|c|c|c|c|c|c|c|c|c|c|c|c|c|c|}
\hline $\begin{array}{c}\mathrm{T} \\
{\left[{ }^{\circ} \mathrm{C}\right]}\end{array}$ & $\begin{array}{c}\mathrm{t} \\
{[\mathrm{h}]}\end{array}$ & $\begin{array}{l}\mathrm{Cu}- \\
\mathrm{Salz} \\
\text { in } \\
\mathrm{mg}\end{array}$ & $\begin{array}{l}\text { NHPI } \\
{[\mathrm{mg}]}\end{array}$ & $\begin{array}{l}\text { NHPI } \\
{[\mathrm{mol} \%]} \\
\text { (soll) }\end{array}$ & $\begin{array}{l}\text { 1-Octen } \\
{[\mathrm{mg}]}\end{array}$ & $\begin{array}{l}n \text {-Nonan } \\
{[\mathrm{mg}]}\end{array}$ & $\begin{array}{l}\text { Gesamt- } \\
\text { menge } \\
\text { gefundene } \\
\text { Produkte } \\
{[\mathrm{mg}]} \\
\end{array}$ & $\begin{array}{l}\text { Verluste } \\
{[\%]}\end{array}$ & $\begin{array}{l}\text { Unver- } \\
\text { brauchtes } \\
\text { 1-Octen } \\
{[\mathrm{mg}]}\end{array}$ & $\begin{array}{l}\text { Umsatz } \\
{[\%]}\end{array}$ & $\begin{array}{l}\text { Epoxid- } \\
\text { ausbeute } \\
{[\mathrm{mg}]}\end{array}$ & $\begin{array}{l}\text { Epoxid- } \\
\text { ausbeute } \\
{[\%]}\end{array}$ & $\begin{array}{l}\text { Selek- } \\
\text { tivität } \\
{[\%]}\end{array}$ \\
\hline 100 & 13 & 25 & 410 & 10 & 2930 & 1480 & 1809 & 38.3 & 1421 & 51.5 & 136 & 4.7 & 9.0 \\
\hline 95 & 14 & 25 & 412 & 10 & 2700 & 1520 & 1695 & 37.2 & 1259 & 53.4 & 160 & 5.9 & 11.1 \\
\hline
\end{tabular}

Molprozent Angaben beziehen sich auf 1-Octen (= $100 \%)$ 


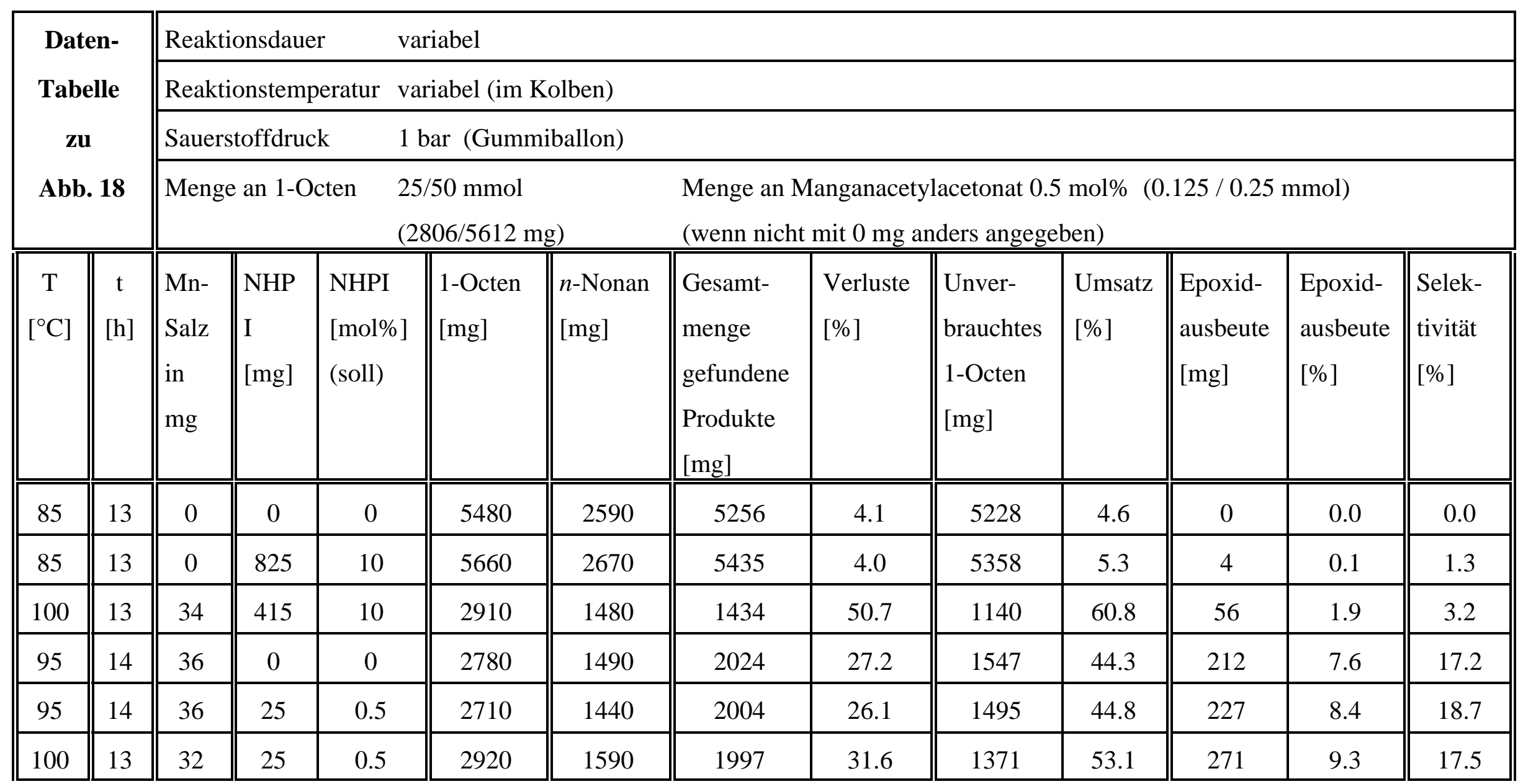

Molprozent Angaben beziehen sich auf 1-Octen (= $100 \%)$ 


\begin{tabular}{|c|c|c|c|c|c|c|c|c|c|c|c|c|c|}
\hline \multirow{4}{*}{\multicolumn{2}{|c|}{$\begin{array}{l}\text { Daten- } \\
\text { Tabelle } \\
\text { zu } \\
\text { Abb. } 19\end{array}$}} & \multicolumn{12}{|c|}{ Reaktionsdauer variabel } \\
\hline & & \multicolumn{12}{|c|}{ Reaktionstemperatur variabel (im Kolben) } \\
\hline & & \multicolumn{5}{|c|}{1 bar (Gummiballon) } & & & & & & & \\
\hline & & \multicolumn{3}{|c|}{ Menge an 1-Octen } & \multicolumn{2}{|c|}{$\begin{array}{l}25 / 50 \mathrm{mmol} \\
(2806 / 5612 \mathrm{mg})\end{array}$} & \multicolumn{7}{|c|}{$\begin{array}{l}\text { Menge an Metallacetylacetonat } 0.5 \mathrm{~mol} \%(0.125 / 0.25 \mathrm{mmol}) \\
\text { (wenn nicht mit } 0 \mathrm{mg} \text { anders angegeben) }\end{array}$} \\
\hline $\begin{array}{c}\mathrm{T} \\
{\left[{ }^{\circ} \mathrm{C}\right]}\end{array}$ & th] & $\begin{array}{l}\text { M- } \\
\text { Salz } \\
\text { in } \\
\text { mg }\end{array}$ & $\begin{array}{l}\text { NHP } \\
\text { I } \\
{[\mathrm{mg}]}\end{array}$ & $\begin{array}{l}\text { NHPI } \\
\text { [mol\%] } \\
\text { (soll) }\end{array}$ & $\begin{array}{l}\text { 1-Octen } \\
{[\mathrm{mg}]}\end{array}$ & $\begin{array}{l}n \text {-Nonan } \\
{[\mathrm{mg}]}\end{array}$ & $\begin{array}{l}\text { Gesamt- } \\
\text { menge } \\
\text { gefundene } \\
\text { Produkte } \\
\text { [mg] }\end{array}$ & $\begin{array}{l}\text { Verluste } \\
{[\%]}\end{array}$ & $\begin{array}{l}\text { Unver- } \\
\text { brauchtes } \\
1-\text { Octen } \\
{[\mathrm{mg}]}\end{array}$ & $\begin{array}{l}\text { Umsatz } \\
{[\%]}\end{array}$ & $\begin{array}{l}\text { Epoxid- } \\
\text { ausbeute } \\
{[\mathrm{mg}]}\end{array}$ & $\begin{array}{l}\text { Epoxid- } \\
\text { ausbeute } \\
{[\%]}\end{array}$ & $\begin{array}{l}\text { Selek- } \\
\text { tivität } \\
{[\%]}\end{array}$ \\
\hline 85 & 13 & 0 & 0 & 0 & 5480 & 2590 & 5256 & 4.1 & 5228 & 4.6 & 0 & 0.0 & 0.0 \\
\hline 100 & 14 & $\begin{array}{l}\mathrm{VO} \\
33\end{array}$ & 0 & 0 & 2860 & 1410 & 2539 & 11.2 & 2525 & 11.7 & 0 & 0.0 & 0.0 \\
\hline 100 & 14 & $\begin{array}{l}\mathrm{Pd} \\
38\end{array}$ & 410 & 10 & 2850 & 1380 & 2551 & 10.5 & 2463 & 13.6 & 0 & 0.0 & 0.0 \\
\hline 85 & 13 & 0 & 825 & 10 & 5660 & 2670 & 5435 & 4.0 & 5358 & 5.3 & 4 & 0.1 & 1.3 \\
\hline
\end{tabular}




\begin{tabular}{|c|c|c|c|c|c|c|c|c|c|c|c|c|c|}
\hline $\begin{array}{c}\mathrm{T} \\
{\left[{ }^{\circ} \mathrm{C}\right]}\end{array}$ & $\begin{array}{c}\mathrm{t} \\
{[\mathrm{h}]}\end{array}$ & $\begin{array}{l}\text { M- } \\
\text { Salz } \\
\text { in } \\
\text { mg }\end{array}$ & $\begin{array}{l}\text { NHP } \\
\text { I } \\
{[\mathrm{mg}]}\end{array}$ & $\begin{array}{l}\text { NHPI } \\
\text { [mol\%] } \\
\text { (soll) }\end{array}$ & $\begin{array}{l}\text { 1-Octen } \\
{[\mathrm{mg}]}\end{array}$ & $\begin{array}{l}n \text {-Nonan } \\
{[\mathrm{mg}]}\end{array}$ & $\begin{array}{l}\text { Gesamt- } \\
\text { menge } \\
\text { gefundene } \\
\text { Produkte } \\
{[\mathrm{mg}]} \\
\end{array}$ & $\begin{array}{l}\text { Verluste } \\
{[\%]}\end{array}$ & $\begin{array}{l}\text { Unver- } \\
\text { brauchtes } \\
1 \text {-Octen } \\
{[\mathrm{mg}]}\end{array}$ & $\begin{array}{l}\text { Umsatz } \\
{[\%]}\end{array}$ & $\begin{array}{l}\text { Epoxid- } \\
\text { ausbeute } \\
{[\mathrm{mg}]}\end{array}$ & $\begin{array}{l}\text { Epoxid- } \\
\text { ausbeute } \\
{[\%]}\end{array}$ & $\begin{array}{l}\text { Selek- } \\
\text { tivität } \\
{[\%]}\end{array}$ \\
\hline 100 & 14 & $\begin{array}{l}\mathrm{Pd} \\
38\end{array}$ & 0 & 0 & 2830 & 1460 & 2614 & 7.6 & 2521 & 10.9 & 5 & 0.2 & 1.7 \\
\hline 100 & 14 & $\begin{array}{l}\text { VO } \\
34\end{array}$ & 408 & 10 & 2810 & 1420 & 1549 & 44.9 & 1411 & 49.8 & 14 & 0.5 & 0.9 \\
\hline 100 & 13 & $\begin{array}{r}\mathrm{Mn} \\
34\end{array}$ & 415 & 10 & 2910 & 1480 & 1434 & 50.7 & 1140 & 60.8 & 56 & 1.9 & 3.2 \\
\hline 95 & 14 & $\begin{array}{c}\text { Mn } \\
36\end{array}$ & 0 & 0 & 2780 & 1490 & 2024 & 27.2 & 1547 & 44.3 & 212 & 7.6 & 17.2 \\
\hline
\end{tabular}

Molprozent Angaben beziehen sich auf 1-Octen (= $100 \%)$ 


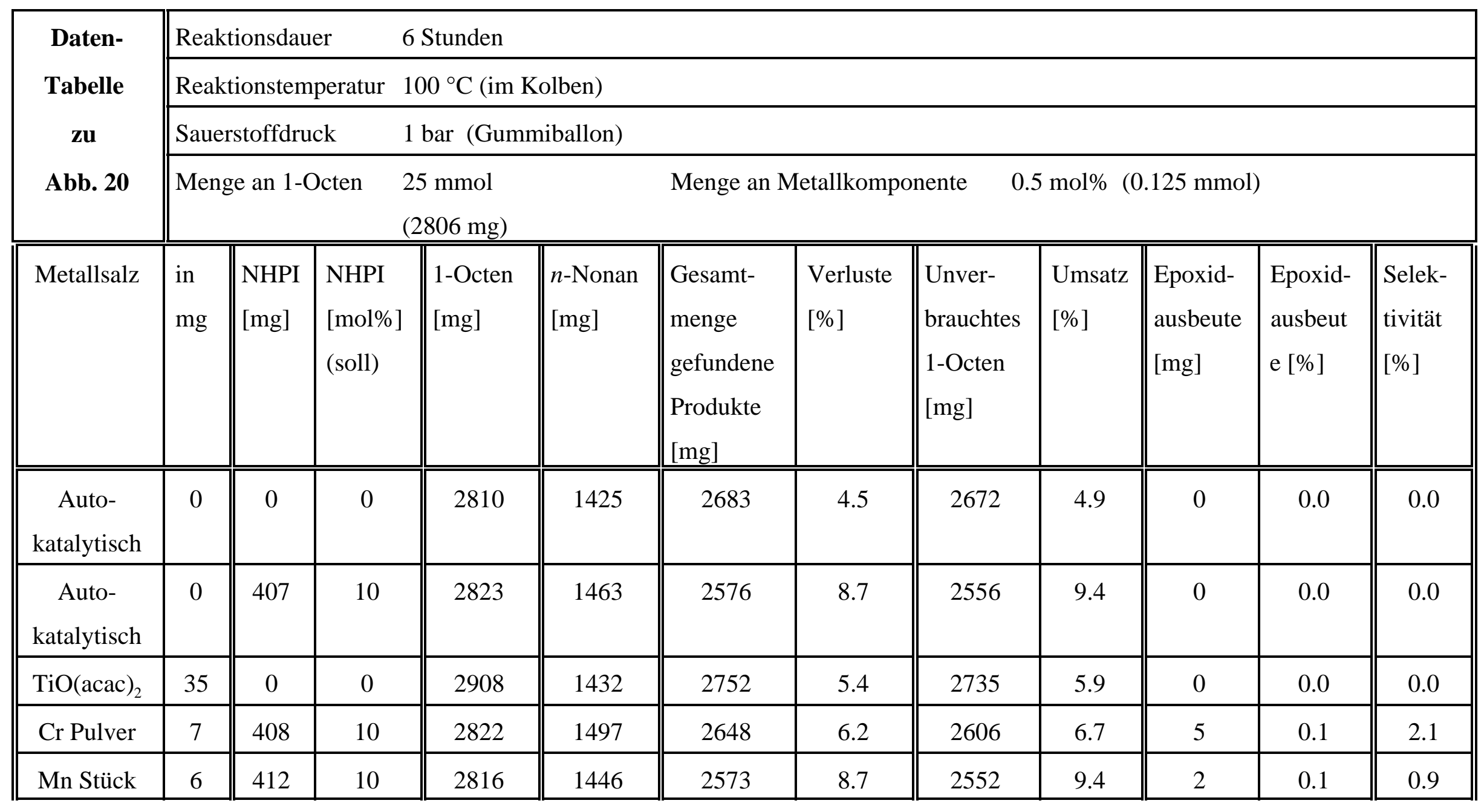




\begin{tabular}{|c|c|c|c|c|c|c|c|c|c|c|c|c|}
\hline Metallsalz & $\begin{array}{l}\text { in } \\
\mathrm{mg}\end{array}$ & $\begin{array}{l}\text { NHPI } \\
{[\mathrm{mg}]}\end{array}$ & $\begin{array}{l}\text { NHPI } \\
{[\mathrm{mol} \%]}\end{array}$ & $\begin{array}{c}\text { 1-Octen } \\
{[\mathrm{mg}]}\end{array}$ & $\begin{array}{c}n \text {-Nonan } \\
{[\mathrm{mg}]}\end{array}$ & $\begin{array}{c}\text { Gesamt- } \\
\text { menge } \\
\text { gefundene } \\
\text { Produkte } \\
\text { [mg] }\end{array}$ & $\begin{array}{c}\text { Verluste } \\
{[\%]}\end{array}$ & $\begin{array}{c}\text { Unver- } \\
\text { brauchtes } \\
\text { 1-Octen } \\
\text { [mg] }\end{array}$ & $\begin{array}{c}\text { Umsatz } \\
{[\%]}\end{array}$ & $\begin{array}{c}\text { Epoxid- } \\
\text { ausbeute } \\
{[\mathrm{mg}]}\end{array}$ & $\begin{array}{c}\text { Epoxid- } \\
\text { ausbeut } \\
\text { e [\%] }\end{array}$ & $\begin{array}{c}\text { Selek- } \\
\text { tivität } \\
{[\%]}\end{array}$ \\
\hline $\mathrm{Cu}$ Pulver & 9 & 0 & 0 & 2787 & 1460 & 2552 & 8.4 & 2475 & 11.2 & 18 & 0.6 & 5.7 \\
\hline Mo Stück & 12 & 411 & 10 & 2828 & 1438 & 2608 & 7.8 & 2544 & 10.1 & 20 & 0.7 & 7.1 \\
\hline $\mathrm{MnO}_{2}$ & 11 & 408 & 10 & 2807 & 1415 & 2533 & 9.8 & 2419 & 13.8 & 22 & 0.8 & 5.8 \\
\hline $\mathrm{Cr}(\mathrm{acac})_{3}$ & 43 & 411 & 10 & 2930 & 1420 & 1962 & 33.1 & 1707 & 41.7 & 33 & 1.1 & 2.7 \\
\hline $\mathrm{TiO}(\mathrm{acac})_{2}$ & 33 & 409 & 10 & 2894 & 1461 & 2497 & 13.7 & 2329 & 19.5 & 58 & 2.0 & 10.4 \\
\hline $\begin{array}{c}\mathrm{Mo}(\mathrm{O})_{2} \\
(\mathrm{acac})_{2}\end{array}$ & 42 & 413 & 10 & 2850 & 1400 & 2007 & 29.6 & 1796 & 37.0 & 67 & 2.4 & 6.4 \\
\hline $\mathrm{MoO}_{3}$ & 19 & 408 & 10 & 2828 & 1417 & 2396 & 15.3 & 2233 & 6.8 & 78 & 2.8 & 13.1 \\
\hline $\mathrm{Co}(\mathrm{acac})_{2}$ & 33 & 410 & 10 & 2859 & 1531 & 1622 & 43.3 & 1323 & 53.7 & 106 & 3.7 & 6.9 \\
\hline $\mathrm{Cu}(\mathrm{acac})_{2}$ & 33 & 406 & 10 & 2760 & 1436 & 2073 & 24.9 & 1763 & 36.1 & 107 & 3.9 & 10.7 \\
\hline $\mathrm{Cu}$ Pulver & 8 & 410 & 10 & 2984 & 1453 & 2371 & 20.5 & 2035 & 31.8 & 124 & 4.2 & 13.0 \\
\hline
\end{tabular}




\begin{tabular}{|c|c|c|c|c|c|c|c|c|c|c|c|c|}
\hline Metallsalz & $\begin{array}{l}\text { in } \\
\mathrm{mg}\end{array}$ & $\begin{array}{l}\text { NHPI } \\
{[\mathrm{mg}]}\end{array}$ & $\begin{array}{l}\text { NHPI } \\
{[\mathrm{mol} \%]}\end{array}$ & $\begin{array}{c}\text { 1-Octen } \\
{[\mathrm{mg}]}\end{array}$ & $\begin{array}{c}n \text {-Nonan } \\
{[\mathrm{mg}]}\end{array}$ & $\begin{array}{c}\text { Gesamt- } \\
\text { menge } \\
\text { gefundene } \\
\text { Produkte } \\
\text { [mg] }\end{array}$ & $\begin{array}{c}\text { Verluste } \\
{[\%]}\end{array}$ & $\begin{array}{c}\text { Unver- } \\
\text { brauchtes } \\
\text { 1-Octen } \\
{[\mathrm{mg}]}\end{array}$ & $\begin{array}{c}\text { Umsatz } \\
{[\%]}\end{array}$ & $\begin{array}{c}\text { Epoxid- } \\
\text { ausbeute } \\
{[\mathrm{mg}]}\end{array}$ & $\begin{array}{c}\text { Epoxid- } \\
\text { ausbeut } \\
\text { e [\%] }\end{array}$ & $\begin{array}{c}\text { Selek- } \\
\text { tivität } \\
{[\%]}\end{array}$ \\
\hline $\begin{array}{c}\mathrm{Co}(\mathrm{OAc})_{2} \\
\cdot 4 \mathrm{H}_{2} \mathrm{O}\end{array}$ & 32 & 412 & 10 & 2810 & 1400 & 1592 & 43.4 & 1317 & 53.1 & 121 & 4.3 & 8.1 \\
\hline $\begin{array}{c}\mathrm{Cu}(\mathrm{OAc})_{2} \\
\cdot \mathrm{H}_{2} \mathrm{O}\end{array}$ & 25 & 409 & 10 & 2840 & 1528 & 1988 & 30.0 & 1610 & 43.3 & 146 & 5.1 & 11.9 \\
\hline $\operatorname{Mn}(\mathrm{acac})_{2}$ & 32 & 413 & 10 & 2840 & 1390 & 1829 & 35.6 & 1413 & 50.2 & 145 & 5.1 & 10.2 \\
\hline $\mathrm{V}_{2} \mathrm{O}_{5}$ & 24 & 411 & 10 & 2830 & 1400 & 1990 & 29.7 & 1607 & 43.2 & 171 & 6.1 & 13.9 \\
\hline $\begin{array}{c}\mathrm{Mn}(\mathrm{OAc})_{2} \\
\cdot 4 \mathrm{H}_{2} \mathrm{O}\end{array}$ & 30 & 410 & 10 & 2820 & 1488 & 2127 & 24.6 & 1693 & 40.0 & 179 & 6.3 & 15.8 \\
\hline
\end{tabular}

Molprozent Angaben beziehen sich auf 1-Octen (= 100 \%) 


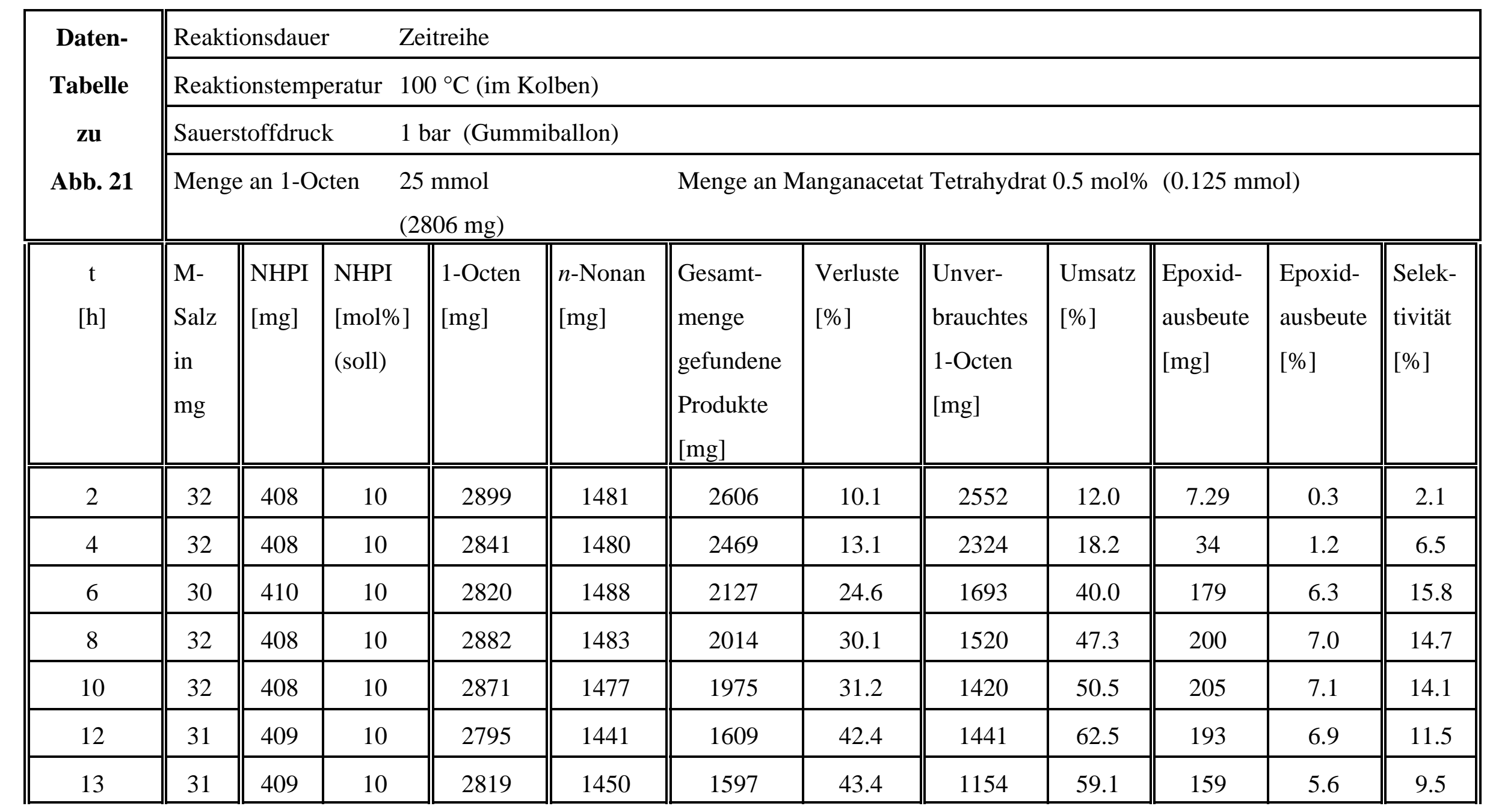




\begin{tabular}{|c|c|c|c|c|c|c|c|c|c|c|c|c|}
\hline $\begin{array}{c}\mathrm{t} \\
{[\mathrm{h}]}\end{array}$ & $\begin{array}{l}\text { M- } \\
\text { Salz } \\
\text { in } \\
\text { mg }\end{array}$ & $\begin{array}{l}\text { NHPI } \\
{[\mathrm{mg}]}\end{array}$ & $\begin{array}{l}\text { NHPI } \\
{[\mathrm{mol} \%]} \\
\text { (soll) }\end{array}$ & $\begin{array}{l}\text { 1-Octen } \\
{[\mathrm{mg}]}\end{array}$ & $\begin{array}{l}n \text {-Nonan } \\
{[\mathrm{mg}]}\end{array}$ & $\begin{array}{l}\text { Gesamt- } \\
\text { menge } \\
\text { gefundene } \\
\text { Produkte } \\
{[\mathrm{mg}]} \\
\end{array}$ & $\begin{array}{l}\text { Verluste } \\
{[\%]}\end{array}$ & $\begin{array}{l}\text { Unver- } \\
\text { brauchtes } \\
1 \text {-Octen } \\
{[\mathrm{mg}]}\end{array}$ & $\begin{array}{l}\text { Umsatz } \\
{[\%]}\end{array}$ & $\begin{array}{l}\text { Epoxid- } \\
\text { ausbeute } \\
{[\mathrm{mg}]}\end{array}$ & $\begin{array}{l}\text { Epoxid- } \\
\text { ausbeute } \\
{[\%]}\end{array}$ & $\begin{array}{l}\text { Selek- } \\
\text { tivität } \\
{[\%]}\end{array}$ \\
\hline 14 & 31 & 408 & 10 & 2850 & 1416 & 1804 & 36.7 & 1164 & 59.2 & 226 & 7.9 & 13.4 \\
\hline 16 & 31 & 408 & 10 & 2781 & 1419 & 1480 & 48.1 & 1044 & 62.5 & 148 & 5.3 & 8.5 \\
\hline
\end{tabular}

Molprozent Angaben beziehen sich auf 1-Octen (=100\%) 


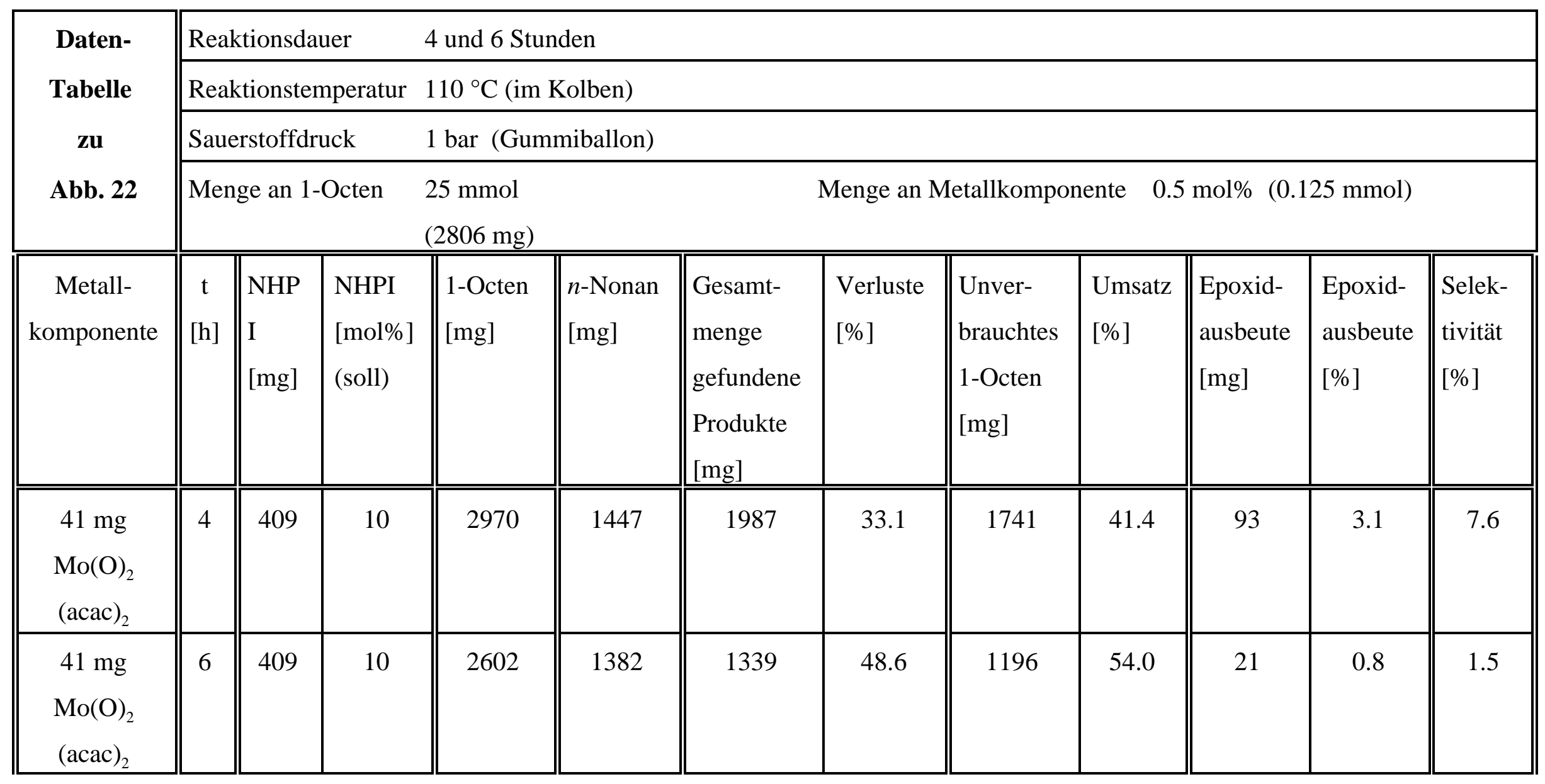




\begin{tabular}{|c|c|c|c|c|c|c|c|c|c|c|c|c|}
\hline $\begin{array}{c}\text { Metall- } \\
\text { komponente }\end{array}$ & $\begin{array}{c}\mathrm{t} \\
{[\mathrm{h}]}\end{array}$ & $\begin{array}{l}\text { NHP } \\
\text { I } \\
{[\mathrm{mg}]}\end{array}$ & $\begin{array}{l}\text { NHPI } \\
{[\mathrm{mol} \%]} \\
\text { (soll) }\end{array}$ & $\begin{array}{l}\text { 1-Octen } \\
{[\mathrm{mg}]}\end{array}$ & $\begin{array}{l}n \text {-Nonan } \\
{[\mathrm{mg}]}\end{array}$ & $\begin{array}{l}\text { Gesamt- } \\
\text { menge } \\
\text { gefundene } \\
\text { Produkte } \\
{[\mathrm{mg}]} \\
\end{array}$ & $\begin{array}{l}\text { Verluste } \\
{[\%]}\end{array}$ & $\begin{array}{l}\text { Unver- } \\
\text { brauchtes } \\
1 \text {-Octen } \\
{[\mathrm{mg}]}\end{array}$ & $\begin{array}{l}\text { Umsatz } \\
{[\%]}\end{array}$ & $\begin{array}{l}\text { Epoxid- } \\
\text { ausbeute } \\
{[\mathrm{mg}]}\end{array}$ & $\begin{array}{l}\text { Epoxid- } \\
\text { ausbeute } \\
{[\%]}\end{array}$ & $\begin{array}{l}\text { Selek- } \\
\text { tivität } \\
{[\%]}\end{array}$ \\
\hline $\begin{array}{c}33 \mathrm{mg} \\
\mathrm{Mn}(\mathrm{acac})_{2}\end{array}$ & 4 & 408 & 10 & 2943 & 1423 & 1714 & 41.8 & 1495 & 49.2 & 52 & 1.8 & 3.6 \\
\hline $\begin{array}{c}33 \mathrm{mg} \\
\mathrm{Mn}(\mathrm{acac})_{2} \\
\end{array}$ & 6 & 409 & 10 & 2788 & 1405 & 1431 & 48.7 & 1225 & 56.1 & 52 & 1.9 & 3.3 \\
\hline $\begin{array}{c}31 \mathrm{mg} \\
\mathrm{Mn}(\mathrm{OAc})_{2} \\
\cdot 4 \mathrm{H}_{2} \mathrm{O}\end{array}$ & 4 & 408 & 10 & 2798 & 1436 & 2133 & 23.8 & 1792 & 35.9 & 136 & 4.9 & 13.5 \\
\hline $\begin{array}{c}31 \mathrm{mg} \\
\mathrm{Mn}(\mathrm{OAc})_{2} \\
\cdot 4 \mathrm{H}_{2} \mathrm{O}\end{array}$ & 6 & 408 & 10 & 2879 & 1437 & 1869 & 35.1 & 1489 & 48.3 & 143 & 5.0 & 10.3 \\
\hline $\begin{array}{r}23 \\
\mathrm{~V}_{2} \mathrm{O}_{5} \\
\end{array}$ & 4 & 409 & 10 & 2996 & 1446 & 2145 & 28.4 & 1715 & 42.8 & 174 & 5.8 & 13.6 \\
\hline
\end{tabular}




\begin{tabular}{|c|c|c|c|c|c|c|c|c|c|c|c|c|}
\hline $\begin{array}{c}\text { Metall- } \\
\text { komponente }\end{array}$ & $\begin{array}{c}\mathrm{t} \\
{[\mathrm{h}]}\end{array}$ & $\begin{array}{l}\text { NHP } \\
\text { I } \\
{[\mathrm{mg}]}\end{array}$ & $\begin{array}{l}\text { NHPI } \\
\text { [mol\%] } \\
\text { (soll) }\end{array}$ & $\begin{array}{l}\text { 1-Octen } \\
{[\mathrm{mg}]}\end{array}$ & $\begin{array}{l}n \text {-Nonan } \\
{[\mathrm{mg}]}\end{array}$ & $\begin{array}{l}\text { Gesamt- } \\
\text { menge } \\
\text { gefundene } \\
\text { Produkte } \\
{[\mathrm{mg}]} \\
\end{array}$ & $\begin{array}{l}\text { Verluste } \\
{[\%]}\end{array}$ & $\begin{array}{l}\text { Unver- } \\
\text { brauchtes } \\
1-\text { Octen } \\
\text { [mg] }\end{array}$ & $\begin{array}{l}\text { Umsatz } \\
{[\%]}\end{array}$ & $\begin{array}{l}\text { Epoxid- } \\
\text { ausbeute } \\
{[\mathrm{mg}]}\end{array}$ & $\begin{array}{l}\text { Epoxid- } \\
\text { ausbeute } \\
{[\%]}\end{array}$ & $\begin{array}{l}\text { Selek- } \\
\text { tivität } \\
{[\%]}\end{array}$ \\
\hline $\begin{array}{l}23 \mathrm{mg} \\
\mathrm{V}_{2} \mathrm{O}_{5}\end{array}$ & 6 & 409 & 10 & 2715 & 1539 & 1503 & 44.6 & 1099 & 59.5 & 160 & 5.9 & 9.9 \\
\hline
\end{tabular}

Molprozent Angaben beziehen sich auf 1-Octen (= $100 \%)$ 


\begin{tabular}{|c|c|c|c|c|c|c|c|c|c|c|c|c|}
\hline Daten- & \multicolumn{12}{|c|}{ Reaktionsdauer $\quad 6$ Stunden } \\
\hline Tabelle & \multicolumn{12}{|c|}{ Reaktionstemperatur $100{ }^{\circ} \mathrm{C}$ (im Kolben) } \\
\hline & \multicolumn{12}{|c|}{ Sauerstoffdruck 1 bar (Gummiballon) } \\
\hline Abb. 23 & \multicolumn{3}{|c|}{ Menge an 1-Octen } & \multicolumn{2}{|c|}{$25 \mathrm{mmol}(2806 \mathrm{mg})$} & \multicolumn{7}{|c|}{ Menge an $\mathrm{Mn}(\mathrm{OAc})_{2} \cdot 4 \mathrm{H}_{2} \mathrm{O}$} \\
\hline $\begin{array}{c}\text { Metallsalz } \\
\mathrm{Mn}(\mathrm{OAc})_{2} \\
\cdot 4 \mathrm{H}_{2} \mathrm{O}\end{array}$ & $\begin{array}{l}\text { in } \\
\mathrm{mg}\end{array}$ & $\begin{array}{l}\text { NHPI } \\
{[\mathrm{mg}]}\end{array}$ & $\begin{array}{l}\text { NHPI } \\
\text { [mol\%] } \\
\text { (soll) }\end{array}$ & $\begin{array}{l}\text { 1-Octen } \\
{[\mathrm{mg}]}\end{array}$ & $\begin{array}{l}n \text {-Nonan } \\
{[\mathrm{mg}]}\end{array}$ & $\begin{array}{l}\text { Gesamt- } \\
\text { menge } \\
\text { gefundene } \\
\text { Produkte } \\
\text { [mg] }\end{array}$ & $\begin{array}{l}\text { Verluste } \\
{[\%]}\end{array}$ & $\begin{array}{l}\text { Unver- } \\
\text { brauchtes } \\
\text { 1-Octen } \\
{[\mathrm{mg}]}\end{array}$ & $\begin{array}{l}\text { Umsatz } \\
{[\%]}\end{array}$ & $\begin{array}{l}\text { Epoxid- } \\
\text { ausbeute } \\
{[\mathrm{mg}]}\end{array}$ & $\begin{array}{l}\text { Epoxid- } \\
\text { ausbeute } \\
{[\%]}\end{array}$ & $\begin{array}{l}\text { Selek- } \\
\text { tivität } \\
{[\%]}\end{array}$ \\
\hline & 0 & 407 & 10 & 2823 & 1463 & 2576 & 8.8 & 2556 & 9.4 & 0 & 0 & 0 \\
\hline & 31 & 21 & 0.5 & 2867 & 1471 & 2435 & 15.3 & 2168 & 24.6 & 103 & 3.6 & 14.7 \\
\hline & 31 & 103 & 2.5 & 2861 & 1496 & 2345 & 18.0 & 2079 & 27.3 & 97 & 3.4 & 12.5 \\
\hline & 31 & 203 & 5 & 2876 & 1512 & 2265 & 21.3 & 1903 & 33.8 & 140 & 4.9 & 14.4 \\
\hline & 31 & 306 & 7.5 & 2924 & 1501 & 2216 & 24.2 & 1792 & 38.7 & 173 & 5.9 & 15.3 \\
\hline & 30 & 410 & 10 & 2820 & 1488 & 2127 & 24.6 & 1693 & 40.0 & 179 & 6.3 & 15.8 \\
\hline & 31 & 614 & 15 & 2867 & 1457 & 2413 & 15.8 & 2210 & 22.9 & 63 & 2.2 & 9.5 \\
\hline
\end{tabular}

Molprozent Angaben beziehen sich auf 1-Octen (= $100 \%)$ 


\begin{tabular}{|c|c|c|c|c|c|c|c|c|c|c|c|c|}
\hline \multirow{4}{*}{$\begin{array}{c}\text { Daten- } \\
\text { Tabelle } \\
\text { zu } \\
\text { Abb. } 24\end{array}$} & \multicolumn{12}{|c|}{ Reaktionsdauer $\quad 6$ Stunden } \\
\hline & \multicolumn{12}{|c|}{ Reaktionstemperatur $100{ }^{\circ} \mathrm{C}$ (im Kolben) } \\
\hline & \multicolumn{3}{|c|}{ Sauerstoffdruck } & \multicolumn{9}{|c|}{1 bar (Gummiballon) } \\
\hline & \multicolumn{3}{|c|}{ Menge an 1-Octen } & \multicolumn{2}{|c|}{$\begin{array}{l}25 \mathrm{mmol} \\
(2806 \mathrm{mg}) \\
\end{array}$} & \multicolumn{7}{|c|}{$\begin{array}{l}\text { Menge an Metallkomponente } \\
\mathrm{Cu}(\mathrm{OAc})_{2} \cdot \mathrm{H}_{2} \mathrm{O} \\
\end{array}$} \\
\hline $\begin{array}{c}\text { Metallsalz } \\
\mathrm{Cu}(\mathrm{OAc})_{2} \\
\cdot \mathrm{H}_{2} \mathrm{O}\end{array}$ & $\begin{array}{l}\text { in } \\
\mathrm{mg}\end{array}$ & $\begin{array}{l}\text { NHPI } \\
{[\mathrm{mg}]}\end{array}$ & $\begin{array}{l}\text { NHPI } \\
\text { [mol\%] } \\
\text { (soll) }\end{array}$ & $\begin{array}{l}\text { 1-Octen } \\
{[\mathrm{mg}]}\end{array}$ & $\begin{array}{l}n \text {-Nonan } \\
{[\mathrm{mg}]}\end{array}$ & $\begin{array}{l}\text { Gesamt- } \\
\text { menge } \\
\text { gefundene } \\
\text { Produkte } \\
{[\mathrm{mg}]} \\
\end{array}$ & $\begin{array}{l}\text { Verluste } \\
{[\%]}\end{array}$ & $\begin{array}{l}\text { Unver- } \\
\text { brauchtes } \\
\text { 1-Octen } \\
{[\mathrm{mg}]}\end{array}$ & $\begin{array}{l}\text { Umsatz } \\
{[\%]}\end{array}$ & $\begin{array}{l}\text { Epoxid- } \\
\text { ausbeute } \\
{[\mathrm{mg}]}\end{array}$ & $\begin{array}{l}\text { Epoxid- } \\
\text { ausbeute } \\
{[\%]}\end{array}$ & $\begin{array}{l}\text { Selek- } \\
\text { tivität } \\
{[\%]}\end{array}$ \\
\hline & 0 & 407 & 10 & 2823 & 1463 & 2576 & 8.8 & 2556 & 9.4 & 0 & 0 & 0 \\
\hline & 25 & 0 & 0 & 2790 & 1469 & 2603 & 6.7 & 2569 & 7.9 & 6 & 0.2 & 2.8 \\
\hline & 22 & 25 & 0.5 & 2775 & 1452 & 2459 & 11.4 & 2371 & 14.6 & 21 & 0.8 & 5.2 \\
\hline & 25 & 103 & 2.5 & 2771 & 1494 & 2237 & 19.3 & 1980 & 28.6 & 105 & 3.8 & 8.2 \\
\hline & 25 & 204 & 5 & 2787 & 1476 & 1973 & 29.2 & 1646 & 40.9 & 121 & 4.3 & 10.6 \\
\hline & 25 & 310 & 7.5 & 2858 & 1478 & 2245 & 21.4 & 1874 & 34.4 & 131 & 4.6 & 13.4 \\
\hline & 25 & 409 & 10 & 2840 & 1528 & 1988 & 30.0 & 1610 & 43.3 & 146 & 5.1 & 11.9 \\
\hline
\end{tabular}




\begin{tabular}{||c|l||l|l|l|l|l|l|l|l|l||l|l||}
\hline $\begin{array}{l}\text { Metallsalz } \\
\mathrm{Cu}(\mathrm{OAc})_{2} \\
\cdot \mathrm{H}_{2} \mathrm{O}\end{array}$ & $\begin{array}{l}\text { in } \\
\mathrm{mg}\end{array}$ & $\begin{array}{l}\text { NHPI } \\
{[\mathrm{mg}]}\end{array}$ & $\begin{array}{l}\text { NHPI } \\
{[\mathrm{mol} \%]} \\
(\mathrm{soll})\end{array}$ & $\begin{array}{l}1-\text { Octen } \\
{[\mathrm{mg}]}\end{array}$ & $\begin{array}{l}n \text {-Nonan } \\
{[\mathrm{mg}]}\end{array}$ & $\begin{array}{l}\text { Gesamt- } \\
\text { menge } \\
\text { gefundene } \\
\text { Produkte } \\
{[\mathrm{mg}]}\end{array}$ & $\begin{array}{l}\text { Verluste } \\
{[\%]}\end{array}$ & $\begin{array}{l}\text { Unver- } \\
\text { brauchtes } \\
\text { 1-Octen } \\
{[\mathrm{mg}]}\end{array}$ & $\begin{array}{l}\text { Umsatz } \\
{[\%]}\end{array}$ & $\begin{array}{l}\text { Epoxid- } \\
\text { ausbeute } \\
{[\mathrm{mg}]}\end{array}$ & $\begin{array}{l}\text { Epoxid- } \\
\text { ausbeute } \\
{[\%]}\end{array}$ \\
\hline \hline & 25 & 508 & 12.5 & 2864 & 1416 & 2057 & 28.2 & 1694 & 40.8 & $\begin{array}{l}\text { Selek- } \\
\text { tivität } \\
{[\%]}\end{array}$ \\
\hline & 26 & 612 & 15 & 2814 & 1482 & 2138 & 24.0 & 1774 & 37.0 & 124 & 4.4 & 11.9 \\
\hline
\end{tabular}

Molprozent Angaben beziehen sich auf 1-Octen (=100\%) 


\section{Kristallographische Daten}

\section{Kristalldaten und Strukturverfeinerung Verbindung 1:}

Strukturkennzeichnung

Summenformel

Molmasse

Temperatur

Wellenlänge

Kristallsystem

Raumgruppe

Zelldimensionen

Zellvolumen

Formeleinheiten pro Zelle Z

Berechnete Dichte

Absorptionskoeffizient

$\mathrm{F}(000)$

Kristallgröße

Gemessener $\theta$-Bereich

Indexbereich

Anzahl der gemessen Reflexe

unabhängige Reflexe

Strukturverfeinerung

Daten/ Restraints/ Parameter

Goodness-of-Fit an $\mathrm{F}^{2}$

Endgültige R-Werte [I>2 $\sigma(\mathrm{I})]$

R-Werte (sämtliche Daten)

Größtes Maximum und Minimum
Roe605

$\mathrm{C}_{42} \mathrm{H}_{73} \mathrm{O}_{11} \mathrm{P}_{3} \mathrm{Ti}_{3}$

990.61

150(2) K

$71.073 \mathrm{pm}$

Orthorhombisch

Pnma

$a=2385.0(5) \mathrm{pm}, \alpha=90^{\circ}$

$b=1482.9(3) \mathrm{pm}, \beta=90^{\circ}$

$c=1357.4(3) \mathrm{pm}, \gamma=90^{\circ}$

$$
4.801(2) \mathrm{nm}^{3}
$$

4

\section{$1.371 \mathrm{Mg} / \mathrm{m}^{3}$}

$0.641 \mathrm{~mm}^{-1}$

2096

$0.50 \times 0.50 \times 0.50 \mathrm{~mm}$

$3.57^{\circ}$ bis $22.54^{\circ}$

$-25 \leq \mathrm{h} \leq 25,-15 \leq \mathrm{k} \leq 15,-14 \leq 1 \leq 14$

9999

$3283\left[\mathrm{R}_{\mathrm{int}}=0.0353\right]$

Vollmatrix Least-Squares an $\mathrm{F}^{2}$

3282 / 3 / 320

0.931

$\mathrm{R} 1=0.0325, \mathrm{wR} 2=0.0845$

$\mathrm{R} 1=0.0393, \mathrm{wR} 2=0.0918$

321 und -257 e. $\mathrm{nm}^{-3}$ 
Modell, welches verwendet wurde, um die verzerrte Struktur der $t$ - $\mathrm{BuP}(\mathrm{O})(\mathrm{O})(\mathrm{OH})$ Gruppe in Verbindung 1 zu verfeinern.

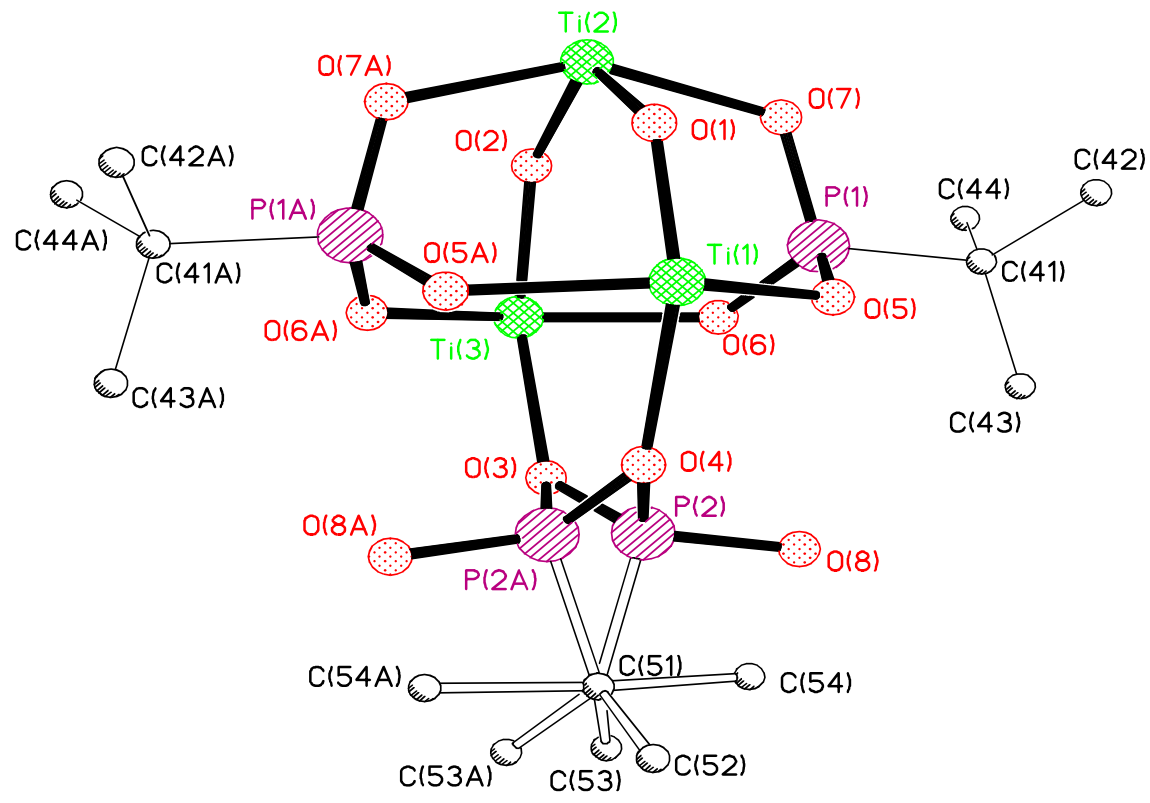

Abb. 1 


\section{Kristalldaten und Strukturverfeinerung Verbindung 2:}

Strukturkennzeichnung

Summenformel

Molmasse

Temperatur

Wellenlänge

Kristallsystem

Raumgruppe

Zelldimensionen

Zellvolumen

Formeleinheiten pro Zelle Z

Berechnete Dichte

Absorptionskoeffizient

$\mathrm{F}(000)$

Kristallgröße

Gemessener $\theta$-Bereich

Indexbereich

Anzahl der gemessen Reflexe

unabhängige Reflexe

Strukturverfeinerung

Daten/ Restraints/ Parameter

Goodness-of-Fit an $\mathrm{F}^{2}$

Endgültige R-Werte [I>2 $\sigma(\mathrm{I})]$

R-Werte (sämtliche Daten)

Größtes Maximum und Minimum
Roe732

$\mathrm{C}_{28} \mathrm{H}_{51} \mathrm{Cl}_{2} \mathrm{O}_{7} \mathrm{P}_{2} \mathrm{Ti}_{2}$

728.33

200(2) K

$71.073 \mathrm{pm}$

Monoklin

$\mathrm{P} 2 / \mathrm{c}$

$a=1117.2(4) \mathrm{pm}, \alpha=90^{\circ}$

$b=1855.8(8) \mathrm{pm}, \beta=93.48(4)^{\circ}$

$c=1702.2(9) \mathrm{pm}, \gamma=90^{\circ}$

$3.523(3) \mathrm{nm}^{3}$

4

$1.373 \mathrm{Mg} / \mathrm{m}^{3}$

$0.736 \mathrm{~mm}^{-1}$

1532

$1.00 \times 0.60 \times 0.50 \mathrm{~mm}$

$3.51^{\circ}$ bis $24.93^{\circ}$

$-13 \leq \mathrm{h} \leq 13,-2 \leq \mathrm{k} \leq 21,-20 \leq 1 \leq 20$

7080

$6100\left[\mathrm{R}_{\mathrm{int}}=0.0728\right]$

Vollmatrix Least-Squares an $\mathrm{F}^{2}$

6088 / 0 / 389

1.058

$\mathrm{R} 1=0.0527, \mathrm{wR} 2=0.1183$

$\mathrm{R} 1=0.0773, \mathrm{wR} 2=0.1406$

468 and -537 e. $\mathrm{nm}^{-3}$ 


\section{Literaturverzeichnis}

[1] Industrielle Organische Chemie, K. Weissermel, H.-J. Arpe, Verlag Chemie Weinheim, New York, 1976.

[2] R. A. Sheldon in The Activation of Dioxygen and Homogeneous Catalytic Oxidation, edited by D. H. R. Barton, A. E. Martell, D. T. Sawyer, Plenum Press, New York, 1993, 9.

[3] W. Pritzkow, R. Radeglia, W Schmidt-Renner, J. prakt. Chem., 1979, 321, 813.

[4] G. Blavoine, D. H. R. Barton, Y. V. Geletii, D. R. Hill in The Activation of Dioxygen and Homogeneous Catalytic Oxidation, edited by D. H. R. Barton, A. E. Martell, D. T. Sawyer, Plenum Press, New York, 1993, 225.

[5] A. E. Martell, R. J. Motekaitis, R. Menif, D. A. Rockcliffe, A. Llobet, J. Mol. Catal. A, 1997, 117, 205.

[6] R. Murugavel, H. W. Roesky, Angew. Chem., 1997, 109, 491; Angew. Chem. Int. Ed. Engl., 1997, 36, 477.

[7] b) M. G. Clerici, G. Belussi, U. Romano, J. Catal., 1991, 129, 159.

a) B. Notari, Catal. Today, 1993, 18, 163.

c) M. G. Clerici, P. Ingallina, J. Catal., 1993, 140, 71.

[8] a) C. Neri, B. Anfossi, A. Esposito, F. Buonomo, EP 100119, 1982; Chem. Abstr., 1984, 101, 38336.

b) M. Padovan, G. Leofanti, P. Roffia, Montedipe S.p.A., EP 0311983, 1989; Chem. Abstr., 1989, 111, 13145.

c) M. Clerici, G. Belussi, Eniricherche S.p.A., EP 0469662, 1991; Chem. Abstr., 1992, 116, 151154. 
d) G. Belussi, M. Clerici, F. Buonomo, R. Franco, U. Romano, A. Esposito, B. Notari, Enichem Sintesi S.p.A., EP200260, 1986; Chem. Abstr., 1987, 106, 158282.

[9] A.-F. Holleman, Lehrbuch der Anorganischen Chemie / HollemanWiberg, 101. Auflage, Walter der Gruyter Verlag Berlin, New York, 1995, $571,581,582$.

[10] a) H. Mimoun, L. Saussine, E. Daire, M. Postel, J. Fischer, R. Weiss, J. Am. Chem. Soc., 1986, 108, 3711.

b) H. Mimoun, M. Mignard, P. Brechot, L. Saussine, J. Am. Chem. Soc., 1983, 105, 3101.

[11] G. Strukul, Catalytic Oxidations with Hydrogenperoxide as Oxidant, Catalysis by Metal Complexes, 9, Kluwer Academic Press, Dordrecht NL, $1992,177$.

[12] Y. Nishida, I. Watanabe, S. Tadahashi, T. Akamatsu, A. Yamazaki, M. Sakamoto, Polyhedron, 1994, 13, 2205.

[13] H. Arzoumanan, A. Blanc, U. Hartig, J. Metzger, Tetrahedron Lett., 1974, $12,1011$.

[14] a) M. Masui, T. Ueshima, S. Ozaki, J. Chem. Soc., Chem. Commun., 1983, 479.

b) M. Masui, S. Hara, T. Ueshima, T. Kawaguchi, S. Ozaki, Chem. Pharm. Bull., 1983, 31, 4209.

c) M. Masui, K. Hosomi, K. Tsuchida, S. Ozaki, Chem. Pharm. Bull., 1985, 33, 4798 .

d) M. Masui, S. Hara, S. Ozaki, Chem. Pharm. Bull., 1986, 34, 975. 
[15] a) C. Ueda, M. Noyama, H. Ohmori, M. Masui, Chem. Pharm. Bull., 1987, 35,3078 .

b) S. Ozaki, T. Hamaguchi, K. Tsuchida, Y. Kimata, M. Masui, J. Chem. Soc., Perkin Trans., 1989, 951.

[16] M. Shimizu, Y. Watanabe, H. Orita, T. Hayakawa, K. Takehira, Bull. Chem. Soc. Jpn., 1993, 66, 251.

[17] a) Y. Ishii, T. Iwahama, S. Sakaguchi, K. Nakayama, Y. Nishiyama, J. Org. Chem., 1996, 61, 4520.

b) Y. Ishii, J. Mol. Catal. A, 1997, 117, 123.

[18] N. Fdil, A. Romane, S. Allaoud, A. Karim,Y. Castanet, A. Mortreux, J. Mol. Catal. A, 1996, 116, 15.

[19] a) C. Ueda, M. Noyama, H. Ohmori, M. Masui, Chem. Pharm. Bull., 1987, $35,1372$.

b) Y. Ishii, T. Iwahama, S. Sakaguchi, K. Nakayama, Y. Nishiyama, J. Org. Chem., 1996, 61, 4520.

c) Y. Ishii, J. Mol. Catal. A, 1997, 117, 123.

[20] a) D. Schnurpfeil, Dissertation „Über die katalysierte Flüssigphasenoxidation von Olefinkohlenwasserstoffen “, 1981, Technische Hochschule „Carl Schorlemmer“, Leuna Merseburg.

b) U. Barth, H. Fiedler, G. Gross, G. Lauterbach, D. Schnurpfeil, J. prakt. Chem., 1981, 323, 887.

c) D. Schnurpfeil, Wiss. Z., TH Leuna Merseburg, 1984, 26, 411; Chem. Abstr., 1984, 101, 190614. 
d) D. Schnurpfeil, Wiss. Z., TH Leuna Merseburg, 1985, 27, 282; Chem. Abstr., 1986, 105, 78449.

e) D. Schnurpfeil, Wiss. Z., TH Leuna Merseburg, 1985, 27, 753; Chem. Abstr., 1987, 106, 66686.

[21] W. Pritzkow, R. Radeglia, W. Schmidt-Renner, J. prakt. Chem., 1979, 321, 813.

[22] H. Trauthwein, Diplomarbeit „Oxovanadium(IV)Komplexe in der Oxidationskatalyse “, 1995, TU München, Arbeitskreis Prof. Dr. Dr. h.c. W. A. Herrmann, S.32 f. .

[23] G. Lauterbach, G. Posselt, R. Schäfer, D. Schnurpfeil, J. prakt. Chem., 1981, 323, 101.

[24] a) U. Barth, H. Fiedler, G. Gross, G. Lauterbach, D. Schnurpfeil, J. prakt. Chem., 1981, 323, 887.

b) W. Pritzkow, R. Radeglia, W. Schmidt-Renner, J. prakt. Chem., 1979, $321,813$.

[25] S. Ozaki, T. Hamaguchi, K. Tsuchida, Y. Kimata, M. Masui, J. Chem. Soc., Perkin Trans. II, 1989, 951.

[26] C. Bianchini, R. W. Zoellner, Adv. Inorg. Chem., 1997, 44, 263.

[27] M. G. Walawalkar, S. Horchler, S. Dietrich, D. Chakraborty, H. W. Roesky, M. Schäfer, H.-G. Schmidt, G. M. Sheldrick, R. Murugavel, Organometallics, 1998, 13, 2865.

[28] a) W. A. Herrmann, M. Elison, J. Fisher, Ch. Köcher, G. R. J. Artus, Angew. Chem., 1995, 107, 2602; Angew. Chem. Int. Ed. Engl., 1995, 34, 2371. 
b) W. A. Herrmann, Ch. Köcher, Angew. Chem., 1997, 109, 2257; Angew. Chem. Int. Ed. Engl., 1997, 36, 2162.

[29] a) T. Wagner-Jauregg, B. E. Hachkley Jr., J. Am. Chem. Soc., 1953, $75,2125$.

b) Th. Rosenberg, Arch. Biochem. , 1964, 105, 315.

c) N. Ranganathan, W. S. Brinigar, J. Org. Chem., 1978, 43, 4853.

d) W. Dabkowski, J. Michalski, C. Radziejewski, Z. Skrypczynski, Chem. Ber., 1982, 115, 1638.

e) W. Dabkowski, J. Michalski, Z. Skrypczynski, Phosphorus and Sulfur, 1986, 26, 321.

[30] H. J. Schönherr, H. W. Wanzlick, Chem. Ber., 1970, 103, 1037.

[31] A. J . Arduengo III, R. L. Harlow, W. J Marshall, T. K. Prakasha, Heteroatom Chemistry, 1996, 7, 421.

[32] Autorenkollektiv, Organikum, 16. Auflage, VEB Deutscher Verlag der Wissenschaften, Berlin, 1986.

[33] a) G.M. Sheldrick, XPREP5, Programm zur Bearbeitung von Beugungsdaten \& Untersuchung reziproker Gitter, V 5.01/486, 1994 Siemens (Teil des Programm Pakets SHELXTL 5.0, Preliminary Release, Siemens Analytical X-ray Instr. Inc.).

b) G.M. Sheldrick, XEMP, Programm zur semiempirischen Absorptionskorrektur, 1988, Universität Göttingen.

c) G.M. Sheldrick, SHELXA-90 V.1.0 F77L, Programm zur empirischen Absorptionskorrektur, 1990, Universität Göttingen. 
d) G.M. Sheldrick, SHELXS-86, Programm zur Strukturlösung, 1986, Universität Göttingen; Acta Crystallogr. Sect. A, 1990, 46, 467. e) G.M. Sheldrick in Crystallographic Computing 6 (Ed.: H.D. Flack, L.K. Simon), IUCr, 1993, Oxford Univ. Press, 100 (SHELXS-92-Erweiterte Version von SHELXS-86).

f) G.M. Sheldrick, SHELXL-93/96/97, Kristallstrukturverfeinerungsprogramm, 1993, 1996, 1997, Universität Göttingen. g) G.M. Sheldrick, XP 5, Programm zur interaktiven, graphischen Darstellung von Molekülen, V 5.03, 1994, Siemens (Teil des Programm Pakets SHELXTL 5.0, Preliminary Release, Siemens Analytical X-ray Instr. Inc.).

[34] a) G. H. Llinás, M. Mena, F. Palacios, P. Royo, R. Serrano, J. Organomet. Chem., 1988, 340, 37.

b) Ibid, 177.

c) M. Mena, P. Royo, R. Serrano, M.A. Pellinghelli, A. Tiripicchio, Organometallics, 1989, 8, 476. 


\section{Stipendien:}

12 ' 95

$01 ' 96-12$ ' 97

Stipendiat der Otto-Vahlbruch Stiftung

Stipendiat der Deutschen Forschungsgemeinschaft im Rahmen des Graduiertenkollegs „Kinetik und Selektivität chemischer Prozesse in verdichteter fluider Phase"

\section{Veröffentlichungen:}

Eamonn F. Murphy, Peihua Yu, Stefan Dietrich, Herbert W. Roesky,* Emilio Parisini and Mathias Noltemeyer:

„Synthesis and Spectroscopic Characterization of a Series of Substituted Cyclopentadienyl Group 4 Fluorides; Crystal Structure of the Acetylacetonato Complex [(acac)2 ( $\left.\left.\eta^{5-}{ }_{5} \mathrm{Me} 5\right) \mathrm{Zr}(\mu-\mathrm{F}) \mathrm{SnMe} 3 \mathrm{Cl}\right] “$. J. Chem. Soc., Dalton Trans., 1996, 1983.

Mrinalini G. Walawalkar, Sabine Horchler, Stefan Dietrich, Debashis Chakraborty, Herbert W. Roesky,* Martina Schäfer, Hans-Georg Schmidt, George M. Sheldrick, and Ramaswamy Murugavel:

„Novel Organic-Soluble Molecular Titanophosphonates with Cage Structures Comparable to Titanium containing Silicates“. Organometallics, 1998, 17, 2865. 


\section{Hochschullehrer:}

Beifuss, Botschwina, Brückner, Buback, Groth, Hack, Hippler, Kahl, Klingebiel, Knepel, Laatsch, Lackner, Luther, de Meijere, Meller, Nölting, Piechulla, Puschmann, Roesky, Schmidt, Schwarzmann, Sheldrick, Susse, Tietze, Treciok, Troe, Wagner, Zeeck. 